\title{
Stereodivergent Synthesis of Chiral Paraconic Acids via Dynamic Kinetic Resolution of 3-Acylsuccinimides
}

\author{
Abhijeet M. Sarkale, Vidyasagar Maurya, Sachin Giri, and Chandrakumar Appayee* \\ Discipline of Chemistry, Indian Institute of Technology Gandhinagar, Palaj, Gandhinagar, Gujarat \\ 382355, India.
}

\section{Supporting information}

\section{Table of Contents}

$\begin{array}{ll}\text { 1. General Information } & \text { S2 }\end{array}$

2. Reaction optimization for the synthesis of 3-acylsuccinimides 10a $\quad$ S2

3. Experimental procedure for the synthesis of 3-acylsuccinimides $\mathbf{1 0} \quad$ S2

4. Experimental procedure for the synthesis of di-succinimides 14a and 14b S5

5. Reaction optimization for the dynamic kinetic resolution of 3-acylsuccinimides 11a

6. Experimental procedure for the dynamic kinetic resolution of 3-acylsuccinimides $11 \quad$ S6

7. Reaction Optimization for the synthesis of trans-paraconic acids 12a $\quad$ S9

8. Experimental procedure for the synthesis of trans-paraconic acids $\mathbf{1 2} \quad \mathrm{S} 10$

9. Experimental procedure for the synthesis of cis-paraconic acids $\mathbf{1 3} \quad \mathrm{S} 12$

10. Experimental procedure for the synthesis of paraconic esters $\mathbf{1 6}$ or $\mathbf{1 7} \quad$ S13

11. Experimental procedure for $\alpha$-methylenation of trans-paraconic acids $\quad S 16$

12. Experimental procedure for $\alpha$-methylation of paraconic acids $\quad S 18$

13. References $\quad$ S19

14. NMR and HPLC data $\quad$ S21 


\section{General Information}

All reactions were carried out in oven-dried glassware unless otherwise noted. Except as otherwise indicated, all reactions were magnetically stirred and monitored by thin-layer chromatography using Merck precoated silica gel plates. Column chromatography was done with 60-120 mesh silica gel supplied by Merck. Commercially available reagents and solvents were used without further purification except as indicated below. Methanol, dichloromethane, and toluene $(\mathrm{PhMe})$ were freshly distilled over calcium hydride under an atmosphere of dry argon prior to use. THF was freshly distilled over sodium under an atmosphere of dry nitrogen prior to use. Organic solutions were concentrated using a Heidolph rotary evaporator. NMR spectra were recorded on a Bruker Advance III $500 \mathrm{MHz}$ spectrometer in chloroform-d. Chemical shifts $(\delta)$ are reported in parts per million (ppm) relative to the internal standard (TMS, $0.00 \mathrm{ppm}$ ). Multiplicities are given as s (singlet), d (doublet), t (triplet), q (quartet), m (multiplet), and br (broad). Specific rotations were measured with a Rudolph Research Analytical polarimeter at $589 \mathrm{~nm}$, and were reported as $[\alpha]_{\mathrm{D}}^{\mathrm{T}}$ ( $c$ in g per $100 \mathrm{~mL}$, solvent, ee). Melting points (m.p.) for solid compounds were recorded using Labindia MR-VIS instrument in open glass capillaries. Chiral HPLC analysis was performed using an Agilent 1200 series and Daicel Chiralpak AD-H, AS-H and Daicel Chiralcel OD-H (Chiral Technologies Eur., $25 \mathrm{~cm} \times 4.6 \mathrm{~mm}$ I.D.) was used. Infrared spectra were taken on a PerkinElmer FTIR spectrometer. The HRMS data for all of the compounds were recorded (in positive ion mode) with a Waters Synapt-G2S ESI-QTOF Mass instrument.

\section{Reaction optimization for the synthesis of 3-acylsuccinimides 10a}

A round-bottomed flask (RBF) equipped with a magnetic stir bar was charged with $\mathrm{N}$ benzylmaleimide $8 \mathbf{a}$ (37 mg, $0.20 \mathrm{mmol}, 1.0$ equiv) and NHC catalyst (0.02 mmol, 0.1 equiv). The RBF was sealed with a rubber septum and purged with nitrogen. Solvent $(2 \mathrm{~mL})$ and base ( $0.02 \mathrm{mmol}, 0.1$ equiv) were added by syringe at room temperature. The reaction was stirred at room temperature for $30 \mathrm{~min}$ and hexanal 9a $(74 \mu \mathrm{L}, 0.60 \mathrm{mmol}, 3.0$ equiv) was added. The reaction mixture was stirred at temperature mentioned in table 1 for $24 \mathrm{~h}$ and quenched by the slow addition of $0.1 \mathrm{M} \mathrm{HCl}(5 \mathrm{~mL})$. The reaction mixture was extracted with $\mathrm{Et}_{2} \mathrm{O}(10 \mathrm{~mL})$ followed by dichloromethane $(10 \mathrm{~mL})$. The combined organic fractions were dried (sodium sulfate) and concentrated in vacuo to get crude product 10a which was further purified by flash column chromatography.

\section{Experimental procedure for the synthesis of 3-acylsuccinimides $\mathbf{1 0}$}

A round-bottomed flask equipped with a magnetic stir bar was charged with maleimide $\mathbf{8}$ (10.0 mmol, 1.0 equiv) and triazolium salt cat-II ${ }^{1}$ ( $0.73 \mathrm{~g}, 2.0 \mathrm{mmol}, 0.2$ equiv). The RBF was sealed with a rubber septum and purged with nitrogen. Toluene $(100 \mathrm{~mL})$ and DIPEA ( $0.35 \mathrm{~mL}, 2.0 \mathrm{mmol}, 0.2$ equiv) were added by syringe at room temperature. The reaction was stirred at room temperature for $30 \mathrm{~min}$ and aldehyde 9 (30.0 mmol, 3.0 equiv) was added. The reaction mixture flask was immediately dipped into preheated oil bath $\left(125{ }^{\circ} \mathrm{C}\right)$. The reaction mixture was refluxed for $24 \mathrm{~h}$ and quenched by the slow addition of $0.1 \mathrm{M} \mathrm{HCl} \mathrm{(80}$ $\mathrm{mL})$. The reaction mixture was extracted with $\mathrm{Et}_{2} \mathrm{O}(40 \mathrm{~mL})$ followed by dichloromethane $(80 \mathrm{~mL})$. The combined organic fractions were dried (sodium sulfate) and concentrated in 
vacuo to get crude product $\mathbf{1 0}$ (along with its enol form in a 4:1 ratio) which was further purified by flash column chromatography.

\section{1-benzyl-3-hexanoylpyrrolidine-2,5-dione (10a)}<smiles>O=C1CC(C(=O)c2ccccc2)C(=O)N1</smiles>

Following the experimental procedure, $N$-benzylmaleimide $8 \mathbf{a}$ (1.87 g, $10.0 \mathrm{mmol}, 1.0$ equiv) and hexanal 9a $(3.7 \mathrm{~mL}, 30 \mathrm{mmol}, 3.0$ equiv) was converted to product $\mathbf{1 0 a}$, which was purified as a colorless oil $(2.5 \mathrm{~g}, 87 \%)$ from the crude reaction mixture using flash column chromatography [Silica gel, hexane/EtOAc (90:10)]. $\mathbf{R}_{f}=0.6$ [hexane/EtOAc (7:3)]. ${ }^{1} \mathbf{H}$ NMR $\left(500 \mathrm{MHz}, \mathrm{CDCl}_{3}\right) \delta 11.20(\mathrm{~s}, 0.2 \mathrm{H}), 7.39-7.25(\mathrm{~m}, 5 \mathrm{H}), 4.64(\mathrm{~d}, J=14.0 \mathrm{~Hz}, 1 \mathrm{H})$, $4.59(\mathrm{~d}, J=14.0 \mathrm{~Hz}, 1 \mathrm{H}), 3.92(\mathrm{dd}, J=8.5,4.0 \mathrm{~Hz}, 0.8 \mathrm{H}), 3.29(\mathrm{dd}, J=18.5,4.5 \mathrm{~Hz}, 0.8 \mathrm{H})$, $3.21(\mathrm{~s}, 0.4 \mathrm{H}), 3.01(\mathrm{dt}, J=7.5,3.0 \mathrm{~Hz}, 0.8 \mathrm{H}), 2.67-2.59(\mathrm{~m}, 1.6 \mathrm{H}), 2.18(\mathrm{t}, J=7.5 \mathrm{~Hz}$, $0.4 \mathrm{H}), 1.63-1.59(\mathrm{~m}, 2 \mathrm{H}), 1.34-1.25(\mathrm{~m}, 4 \mathrm{H}), 0.88(\mathrm{t}, J=7.0 \mathrm{~Hz}, 3 \mathrm{H}) .{ }^{13} \mathbf{C}$ NMR $(125 \mathrm{MHz}$, $\left.\mathrm{CDCl}_{3}\right) \delta 201.8,175.2,174.5,173.8,172.5,170.5,135.9,135.3,128.8,128.7,128.7,128.6$, $128.1,95.3,53.0,43.1,42.8,41.9,32.8,31.7,31.4,31.1,30.0,25.6,22.9,22.4,13.9$. IR (neat) $v$ 2931, 2871, 1777, 1697, 1586, 1394, 1165, $697 \mathrm{~cm}^{-1}$. HRMS (ESI) m/z calcd for $\mathrm{C}_{17} \mathrm{H}_{22} \mathrm{NO}_{3}^{+}[\mathrm{M}+\mathrm{H}]^{+}$288.1594, found 288.1599.

\section{1-benzyl-3-dodecanoylpyrrolidine-2,5-dione (10b)}

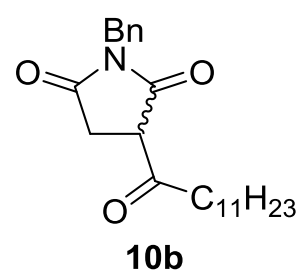

Following the experimental procedure, $N$-benzylmaleimide $8 \mathbf{a}(1.87 \mathrm{~g}, 10.0 \mathrm{mmol}, 1.0$ equiv) and dodecanal $\mathbf{9 b}(5.53 \mathrm{~g}, 30.0 \mathrm{mmol}, 3.0$ equiv) was converted to product $\mathbf{1 0 b}$, which was purified as an brownish solid $(3.34 \mathrm{~g}, 90 \%)$ from the crude reaction mixture using flash column chromatography [Silica gel, hexane/EtOAc (90:10)]. $\mathbf{R}_{f}=0.6$ [hexane/EtOAc (7:3)]. M. P.: $45-47{ }^{\circ} \mathrm{C} .{ }^{1} \mathbf{H}$ NMR $\left(500 \mathrm{MHz}, \mathrm{CDCl}_{3}\right) \delta 11.20(\mathrm{~s}, 0.2 \mathrm{H}), 7.40-7.27(\mathrm{~m}, 5 \mathrm{H}), 4.64(\mathrm{~d}$, $J=14.5 \mathrm{~Hz}, 1 \mathrm{H}), 4.60(\mathrm{~d}, J=14.5 \mathrm{~Hz}, 1 \mathrm{H}), 3.94-3.91(\mathrm{~m}, 0.8 \mathrm{H}), 3.32-3.30(\mathrm{~m}, 0.8 \mathrm{H}), 3.21$ $(\mathrm{s}, 0.4 \mathrm{H}), 3.01(\mathrm{dt}, J=7.5,3.0 \mathrm{~Hz}, 0.8 \mathrm{H}), 2.68-2.59(\mathrm{~m}, 1.6 \mathrm{H}), 2.18(\mathrm{t}, J=7.5 \mathrm{~Hz}, 0.4 \mathrm{H})$, $1.61-1.58(\mathrm{~m}, 2 \mathrm{H}), 1.31-1.26(\mathrm{~m}, 16 \mathrm{H}), 0.88(\mathrm{t}, J=6.5 \mathrm{~Hz}, 3 \mathrm{H}) .{ }^{13} \mathbf{C}$ NMR $(125 \mathrm{MHz}$, $\left.\mathrm{CDCl}_{3}\right) \delta 201.8,175.2,174.5,172.5,172.5,170.5,135.9,135.3,128.8,128.7,128.7,128.6$, 128.1, 127.9, 95.3, 53.0, 43.2, 42.8, 41.9, 31.7, 31.9, 30.0, 29.6, 29.6, 29.4, 29.4, 29.3, 29.3, 29.0, 25.9, 23.3, 22.7, 14.1. IR (neat) $v 2923,2853,1777,1699,1647,1496,1394,1164,697$ $\mathrm{cm}^{-1}$. HRMS (ESI) m/z calcd for $\mathrm{C}_{23} \mathrm{H}_{34} \mathrm{NO}_{3}{ }^{+}[\mathrm{M}+\mathrm{H}]^{+} 372.2533$, found 372.2536.

\section{1-benzyl-3-tetradecanoylpyrrolidine-2,5-dione (10c)}


<smiles>O=C1CC(=O)C(C(=O)O)C1</smiles>

Following the experimental procedure, $N$-benzylmaleimide $8 \mathbf{a}(1.87 \mathrm{~g}, 10.0 \mathrm{mmol}, 1.0$ equiv) and tetradecanal 9c $(6.37 \mathrm{~g}, 30.0 \mathrm{mmol}, 3.0$ equiv) was converted to product 10c, which was purified as brown solid (3.55 g, 89\%) from the crude reaction mixture using flash column chromatography [Silica gel, hexane/EtOAc (90:10)]. $\mathbf{R}_{f}=0.5$ [hexane/EtOAc (8:2)]. M. P.: 55-60 ${ }^{\circ} \mathrm{C} .{ }^{1} \mathbf{H}$ NMR $\left(500 \mathrm{MHz}, \mathrm{CDCl}_{3}\right) \delta 11.20(\mathrm{~s}, 0.2 \mathrm{H}), 7.38-7.25(\mathrm{~m}, 5 \mathrm{H}), 4.64$ (d, $J=$ $14.5 \mathrm{~Hz}, 1 \mathrm{H}), 4.60(\mathrm{~d}, J=14.5 \mathrm{~Hz}, 1 \mathrm{H}), 3.92(\mathrm{dd}, J=9.0,4.5 \mathrm{~Hz}, 0.8 \mathrm{H}), 3.30(\mathrm{dd}, J=18.5$, $4.0 \mathrm{~Hz}, 0.8 \mathrm{H}), 3.21(\mathrm{~s}, 0.4 \mathrm{H}), 3.01(\mathrm{dt}, J=7.5,3.0 \mathrm{~Hz}, 0.8 \mathrm{H}), 2.68-2.60(\mathrm{~m}, 1.6 \mathrm{H}), 2.18(\mathrm{t}, J$ $=7.0 \mathrm{~Hz}, 0.4 \mathrm{H}), 1.61-1.58(\mathrm{~m}, 2 \mathrm{H}), 1.28-1.25(\mathrm{~m}, 20 \mathrm{H}), 0.88(\mathrm{t}, J=7.0 \mathrm{~Hz}, 3 \mathrm{H}) .{ }^{13} \mathbf{C ~ N M R}$ $\left(125 \mathrm{MHz} \mathrm{CDCl}_{3}\right) \delta 201.8,175.2,174.5,173.8,172.5,170.5,135.9,135.3,128.8,128.7$, 128.7, 128.6, 128.1, 127.9, 95.3, 53.0, 43.2, 42.8, 41.9, 31.9, 31.6, 30.0, 29.7, 29.6, 29.6, 29.4, 29.4, 29.3, 29.0, 23.3, 22.7, 14.1. IR (neat) v 2923, 2853, 1701, 1433, 1395, 1346, 1166, 1082, 698, $631 \mathrm{~cm}^{-1}$. HRMS (ESI) m/z calcd for $\mathrm{C}_{25} \mathrm{H}_{38} \mathrm{NO}_{3}{ }^{+}[\mathrm{M}+\mathrm{H}]^{+} 400.2846$, found 400.2855 .

\section{3-hexanoylpyrrolidine-2,5-dione (10d)}<smiles>O=C1CC(C(=O)O)C(=O)N1</smiles>

Following the experimental procedure, maleimide $\mathbf{8 b}(0.97 \mathrm{~g}, 10 \mathrm{mmol}, 1.0$ equiv) and hexanal 9a (3.7 mL, $30 \mathrm{mmol}, 3.0$ equiv) was converted to product 10d, which was purified as brown solid $(1.80 \mathrm{~g}, 91 \%)$ from the crude reaction mixture using flash column chromatography [Silica gel, hexane/EtOAc (70:30)]. $\mathbf{R}_{f}=0.5$ [hexane/EtOAc (1:1)]. M. P.: 54-56 ${ }^{\circ} \mathrm{C} .{ }^{1} \mathbf{H}$ NMR $\left(500 \mathrm{MHz}, \mathrm{CDCl}_{3}\right) \delta 11.14(\mathrm{~s}, 0.2 \mathrm{H}), 8.38(\mathrm{bs}, 1 \mathrm{H}), 3.98(\mathrm{dd}, J=9.0,4.5$ $\mathrm{Hz}, 0.8 \mathrm{H}), 3.34(\mathrm{dd}, J=18.5,4.5 \mathrm{~Hz}, 0.8 \mathrm{H}), 3.25(\mathrm{~s}, 0.4 \mathrm{H}), 3.01(\mathrm{dt}, J=7.0,3.5 \mathrm{~Hz}, 0.8 \mathrm{H})$, 2.73-2.59 (m, 1.6H), $2.20(\mathrm{t}, J=7.0 \mathrm{~Hz}, 0.4 \mathrm{H}), 1.70-1.59(\mathrm{~m}, 2 \mathrm{H}), 1.37-1.26(\mathrm{~m}, 4 \mathrm{H}), 0.89$ $(\mathrm{t}, J=6.5 \mathrm{~Hz}, 3 \mathrm{H}) .{ }^{13} \mathbf{C} \mathbf{N M R}\left(125 \mathrm{MHz}, \mathrm{CDCl}_{3}\right) \delta 201.3,175.8,175.0,174.4,172.8,171.7$, 96.0, 54.3, 43.0, 32.9, 31.3, 31.1, 31.1, 25.5, 22.9, 22.4, 22.4, 13.8. IR (neat) v 3140, 3064, $2929,1782,1751,1725,1681,1339,797 \mathrm{~cm}^{-1}$. HRMS (ESI) $\mathrm{m} / \mathrm{z}$ calcd for $\mathrm{C}_{10} \mathrm{H}_{16} \mathrm{NO}_{3}{ }^{+}[\mathrm{M}+$ $\mathrm{H}]^{+}$198.1125, found 198.1126 .

\section{3-tetradecanoylpyrrolidine-2,5-dione (10e)}<smiles>O=C1CC(C(=O)O)C(=O)N1</smiles> 
Following the experimental procedure, maleimide $\mathbf{8 b}(0.97 \mathrm{~g}, 10 \mathrm{mmol}, 1.0$ equiv) and tetradecanal 9c $(6.37 \mathrm{~g}, 30.0 \mathrm{mmol}, 3.0$ equiv) was converted to product 10e, which was purified as a white solid $(2.47 \mathrm{~g}, 80 \%)$ from the crude reaction mixture using flash column chromatography [Silica gel, hexane/EtOAc (70:30)]. $\mathbf{R}_{f}=0.5$ [hexane/EtOAc (1:1)]. M. P.: 88-92 ${ }^{\circ} \mathrm{C} .{ }^{1} \mathbf{H}$ NMR $\left(500 \mathrm{MHz}, \mathrm{CDCl}_{3}\right) \delta 11.14$ (s, 0.2H), 8.46 (bs, $\left.1 \mathrm{H}\right), 3.97$ (dd, $J=9.0$, $4.5 \mathrm{~Hz}, 0.8 \mathrm{H}), 3.35(\mathrm{dd}, J=18.5,4.5 \mathrm{~Hz}, 0.8 \mathrm{H}), 3.25(\mathrm{~s}, 0.4 \mathrm{H}), 3.01(\mathrm{dt}, J=7.0,3.0 \mathrm{~Hz}$, $0.8 \mathrm{H}), 2.72-2.59(\mathrm{~m}, 1.6 \mathrm{H}), 2.19(\mathrm{t}, J=7.5 \mathrm{~Hz}, 0.4 \mathrm{H}), 1.61-1.59(\mathrm{~m}, 2 \mathrm{H}), 1.29-1.25(\mathrm{~m}$, 20H), $0.87(\mathrm{t}, J=7.0 \mathrm{~Hz}, 3 \mathrm{H}) .{ }^{13} \mathbf{C}$ NMR $\left(125 \mathrm{MHz}, \mathrm{CDCl}_{3}\right) \delta 201.3,175.7,174.9,174.3$, 172.7, 171.7, 96.0, 54.2, 43.0, 32.9, 32.7, 31.9, 31.1, 29.7, 29.6, 29.6, 29.4, 29.3, 29.2, 29.0, 25.8, 23.3, 22.7, 14.1. IR (neat) v 3146, 3150, 2916, 2849, 1779, 1745, 1725, 1689, 1403, 1349, 1211, 1171, 798, 717, $688 \mathrm{~cm}^{-1}$. HRMS (ESI) $\mathrm{m} / \mathrm{z}$ calcd for $\mathrm{C}_{18} \mathrm{H}_{32} \mathrm{NO}_{3}{ }^{+}[\mathrm{M}+\mathrm{H}]^{+}$ 310.2377, found 310.2364.

\section{Experimental procedure for the synthesis of di-succinimides $14 \mathrm{a}$ and $14 \mathrm{~b}$}

A round-bottomed flask equipped with a magnetic stir bar was charged with $\mathrm{N}$ benzylmaleimide 8a (187 mg, $1.0 \mathrm{mmol}, 1.0$ equiv), $\mathrm{K}_{2} \mathrm{CO}_{3}$ (14 mg, $0.10 \mathrm{mmol}, 0.1$ equiv) and triazolium salt cat-II (36 mg, $0.10 \mathrm{mmol}, 0.1$ equiv). The RBF was sealed with a rubber septum and purged with nitrogen. THF $(10 \mathrm{~mL})$ was added by syringe at room temperature. The reaction was stirred at room temperature for $30 \mathrm{~min}$ and hexanal $(369 \mu \mathrm{L} 3.0 \mathrm{mmol}, 3.0$ equiv) was added. The reaction mixture was stirred at $\mathrm{rt}$ for $24 \mathrm{~h}$ and quenched by the slow addition of $0.1 \mathrm{M} \mathrm{HCl}(10 \mathrm{~mL})$. The reaction mixture was extracted with $\mathrm{Et}_{2} \mathrm{O}(10 \mathrm{~mL})$ followed by dichloromethane $(10 \mathrm{~mL})$. The combined organic fractions were dried (sodium sulfate) and concentrated in vacuo to get crude product. The crude product was purified by column chromatography on silica gel [hexane/EtOAc (70:30)] to yield the major diastereomer 14a (114 mg, 48\%) and minor diastereomer 14b (57 mg, 24\%).

1,1'-dibenzyl-3-hexanoyl-[3,3'-bipyrrolidine]-2,2',5,5'-tetraone diastereomer)

$(14 a$,

major

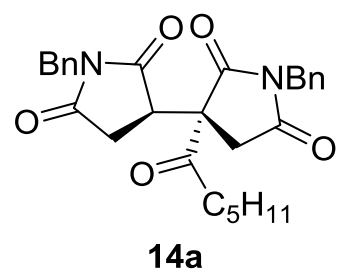

$\mathbf{R}_{\boldsymbol{f}}=0.5$ [hexane/EtOAc (6:4)]. ${ }^{\mathbf{1}} \mathbf{H}$ NMR (500 MHz, $\left.\mathrm{CDCl}_{3}\right) \delta 7.31-7.25(\mathrm{~m}, 10 \mathrm{H}), 4.59(\mathrm{~d}$, $J=14.0 \mathrm{~Hz}, 1 \mathrm{H}), 4.54(\mathrm{~d}, J=14.0 \mathrm{~Hz}, 1 \mathrm{H}), 4.46(\mathrm{~d}, J=14.5 \mathrm{~Hz}, 1 \mathrm{H}), 4.45(\mathrm{~d}, J=14.0 \mathrm{~Hz}$, $1 \mathrm{H}), 3.68(\mathrm{dd}, J=9.5,5.5 \mathrm{~Hz}, 1 \mathrm{H}), 3.04(\mathrm{~d}, J=18.5 \mathrm{~Hz}, 2 \mathrm{H}), 2.98(\mathrm{~d}, J=17.5 \mathrm{~Hz}, 2 \mathrm{H}), 2.95$ $(\mathrm{dd}, J=18.5,9.5 \mathrm{1H}), 2.65(\mathrm{dd}, J=18.5,5.0 \mathrm{~Hz}, 1 \mathrm{H}), 2.37-2.34(\mathrm{~m}, 2 \mathrm{H}), 1.56-1.44(\mathrm{~m}, 2 \mathrm{H})$, 1.21-1.17 (m, 2H), 1.10-1.06 (m, 2H), $0.83(\mathrm{t}, J=7.5,3 \mathrm{H}) .{ }^{13} \mathbf{C ~ N M R}\left(125 \mathrm{MHz}, \mathrm{CDCl}_{3}\right) \delta$ 202.1, 176.4, 174.4, 174.3, 173.3, 135.3, 134.9, 128.8, 128.7, 128.7, 128.3, 128.2, 61.0, 43.0, 42.6, 42.1, 38.5, 35.9, 32.5, 30.9, 22.9, 22.2, 13.8. IR (neat) v 2955, 2931, 1778, 1698, 1586, 1396, 1343, 1167, $701 \mathrm{~cm}^{-1}$. HRMS (ESI) m/z calcd for $\mathrm{C}_{28} \mathrm{H}_{31} \mathrm{~N}_{2} \mathrm{O}_{5}{ }^{+}[\mathrm{M}+\mathrm{H}]^{+} 475.2227$, found 475.2260 . 

diastereomer)<smiles></smiles>

$\mathbf{R}_{f}=0.4$ [hexane/EtOAc (6:4)]. ${ }^{1} \mathbf{H}$ NMR (500 MHz, $\left.\mathrm{CDCl}_{3}\right) \delta 7.39-7.27$ (m, 10H), 4.79 (d, $J$ $=14.0 \mathrm{~Hz}, 1 \mathrm{H}), 4.69(\mathrm{~d}, J=13.5 \mathrm{~Hz}, 1 \mathrm{H}), 4.69(\mathrm{~d}, J=14.0 \mathrm{~Hz}, 1 \mathrm{H}), 4.61(\mathrm{~d}, J=14.5 \mathrm{~Hz}$, $1 \mathrm{H}), 3.38(\mathrm{t}, J=2.5 \mathrm{~Hz}, 1 \mathrm{H}), 3.11(\mathrm{~d}, J=18.5 \mathrm{~Hz}, 1 \mathrm{H}), 2.89-2.83(\mathrm{~m}, 2 \mathrm{H}), 2.61$ (dd, $J=$ $18.5,6.0 \mathrm{~Hz}, 1 \mathrm{H}), 2.34-2.30(\mathrm{~m}, 1 \mathrm{H}), 2.09-2.04(\mathrm{~m}, 1 \mathrm{H}), 1.39-1.43(\mathrm{~m}, 2 \mathrm{H}), 1.18-1.14$ $(\mathrm{m}, 2 \mathrm{H}), 1.04-1.00(\mathrm{~m}, 2 \mathrm{H}), 0.82(\mathrm{t}, J=7.5 \mathrm{~Hz}, 3 \mathrm{H}) .{ }^{13} \mathbf{C} \mathbf{N M R}\left(125 \mathrm{MHz}, \mathrm{CDCl}_{3}\right) \delta 202.1$, 176.1, 174.6, 174.1, 173.5, 135.4, 134.9, 128.8, 128.8, 128.7, 128.7, 128.3, 128.1, 61.6, 43.3, 43.1, 42.7, 37.8, 35.7, 32.2, 30.8, 23.0, 22.2, 13.8. IR (neat) $v 2955,2930,1777,1700,1397$, 1293, 1170, $701 \mathrm{~cm}^{-1}$. HRMS (ESI) $\mathrm{m} / \mathrm{z}$ calcd for $\mathrm{C}_{28} \mathrm{H}_{31} \mathrm{~N}_{2} \mathrm{O}_{5}{ }^{+}[\mathrm{M}+\mathrm{H}]^{+} 475.2227$, found 475.2249 .

\section{Reaction optimization for the dynamic kinetic resolution of 3-acylsuccinimides 11a}

A round-bottomed flask equipped with a magnetic stir bar was charged with 3acylsuccinimide 10a (57 mg, $0.20 \mathrm{mmol}, 1.0$ equiv) and $\mathrm{RuCl}[(S, S)$-Tsdpen $]\left(\eta^{6}\right.$-arene) $(0.01$ mmol, 0.05 equiv). The flask was sealed with a rubber septum and purged with nitrogen. Solvent $(2 \mathrm{~mL})$ followed $\mathrm{HCO}_{2} \mathrm{H} / \mathrm{Et}_{3} \mathrm{~N}(5: 2$ molar ratio) azeotrope $(188 \mu \mathrm{L})$ were added to the reaction mixture at room temperature. The reaction mixture was allowed to stir at temperature mentioned in table 2 , after which it was quenched with $1 \mathrm{M} \mathrm{HCl}(5 \mathrm{~mL})$ and diluted with water $(5 \mathrm{~mL})$. The mixture was extracted with $\mathrm{Et}_{2} \mathrm{O}(10 \mathrm{~mL} X 2)$, organic fraction was dried (sodium sulfate) and concentrated in vacuo to get crude product 11a which was further purified by flash column chromatography.

\section{Experimental procedure for the dynamic kinetic resolution of 3-acylsuccinimides 11}

A round-bottomed flask equipped with a magnetic stir bar was charged with 3acylsuccinimide 10 (8.5 mmol, 1.0 equiv) and $\operatorname{RuCl}[(S, S)$-Tsdpen](p-cymene) (108 mg, 0.021 mmol, 0.02 equiv). The flask was sealed with a rubber septum and purged with nitrogen. DMF $(21 \mathrm{~mL})$ followed $\mathrm{HCO}_{2} \mathrm{H} / \mathrm{Et}_{3} \mathrm{~N}(5: 2$ molar ratio) azeotrope $(8 \mathrm{~mL})$ were added to the reaction mixture at room temperature. The reaction mixture was allowed to stir at $\mathrm{rt}$ for $18 \mathrm{~h}$, after which it was quenched with $1 \mathrm{M} \mathrm{HCl}(25 \mathrm{~mL})$ and diluted with water (80

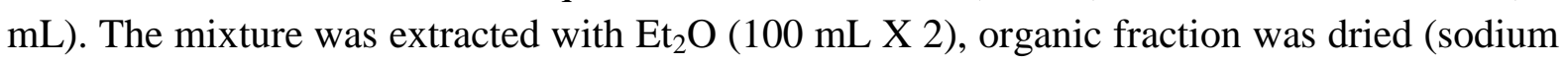
sulfate) and concentrated in vacuo to get crude product 11 which was further purified by flash column chromatography.

\section{Alcohol 11a and 15a}

Following the experimental procedure, 3-acylsuccinimide 10a (2.44 g, $8.5 \mathrm{mmol}, 1.0$ equiv) and $\operatorname{RuCl}[(S, S)$-Tsdpen $](p$-cymene) $(108 \mathrm{mg}, 0.021 \mathrm{mmol}, 0.02$ equiv) was converted to 
product 11a and 15a, which were purified from the crude reaction mixture using flash column chromatography [Silica gel, hexane/EtOAc (85:15)].

(S)-1-benzyl-3-((S)-1-hydroxyhexyl)pyrrolidine-2,5-dione (11a, major diastereomer)

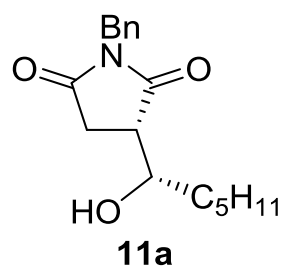

White solid, yield (isolated as a single diastereomer, $1.92 \mathrm{~g}, 78 \%$ ). $\mathbf{R}_{f}=0.4$ [hexane/EtOAc (6:4)]. HPLC analysis Daicel Chiralcel OD-H, $4.6 \mathrm{~mm} \times 250 \mathrm{~mm}($ hex/IPA $=90: 10,0.5$ $\mathrm{mL} / \mathrm{min}, 210 \mathrm{~nm}), \mathrm{t}_{\mathrm{R}}($ minor $)=31.8 \mathrm{~min}, \mathrm{t}_{\mathrm{R}}$ (major) $=35.0 \mathrm{~min}, 97 \%$ ee. $[\boldsymbol{\alpha}]_{\mathrm{D}}{ }^{24}=+9.63(c=$ 1.0, $\mathrm{CHCl}_{3}$ for $97 \%$ ee $)$. M. P.: $71-73{ }^{\circ} \mathrm{C}^{\mathbf{1}}{ }^{\mathbf{H}} \mathbf{N M R}\left(500 \mathrm{MHz}, \mathrm{CDCl}_{3}\right) \delta 7.37-7.27$ (m, 5H), $4.65(\mathrm{~s}, 2 \mathrm{H}), 3.85-3.78(\mathrm{~m}, 1 \mathrm{H}), 3.07(\mathrm{~d}, J=2.5 \mathrm{~Hz}, 1 \mathrm{H}), 2.87-2.84(\mathrm{~m}, 1 \mathrm{H}), 2.80(\mathrm{dd}, J=$ $18.0,5.0 \mathrm{~Hz}, 1 \mathrm{H}), 2.50(\mathrm{dd}, J=18.0,4.5 \mathrm{~Hz}, 1 \mathrm{H}), 1.57-1.54(\mathrm{~m}, 3 \mathrm{H}), 1.53-1.49(\mathrm{~m}, 1 \mathrm{H})$, $1.33-1.25(\mathrm{~m}, 4 \mathrm{H}), 0.88(\mathrm{t}, J=7.0 \mathrm{~Hz}, 3 \mathrm{H}) .{ }^{13} \mathbf{C ~ N M R}\left(125 \mathrm{MHz}, \mathrm{CDCl}_{3}\right) \delta 179.1,175.7$, 135.6, 128.7, 128.0, 72.1, 45.1, 42.4, 34.5, 32.0, 31.6, 24.9, 22.5, 14.0. IR (neat) $v 3455$, 2929, 2858, 1771, 1696, 1400, 1172, $697 \mathrm{~cm}^{-1}$. HRMS (ESI) m/z calcd for $\mathrm{C}_{17} \mathrm{H}_{24} \mathrm{NO}_{3}{ }^{+}[\mathrm{M}+$ $\mathrm{H}]^{+} 290.1751$, found 290.1757 .

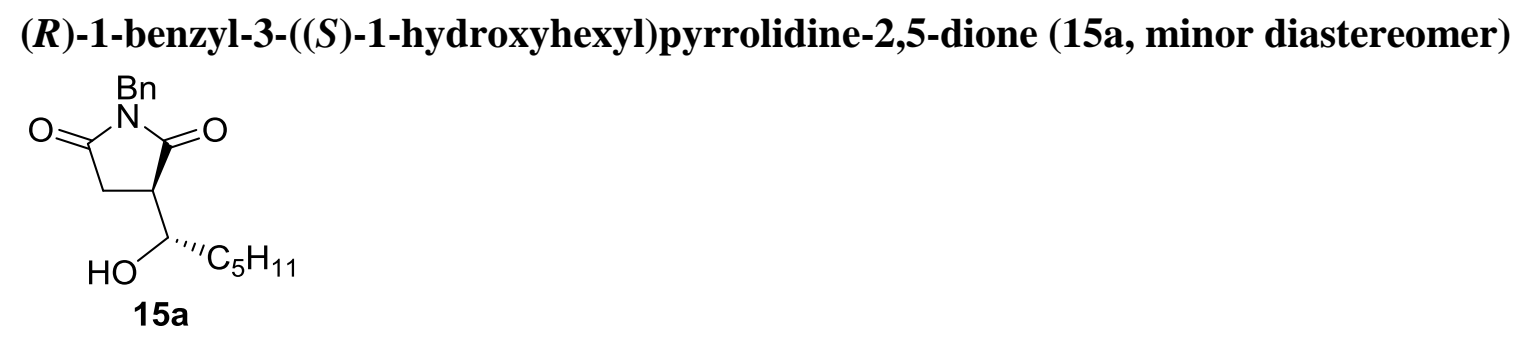

White solid, yield (55 mg, 9\%): $\mathbf{R}_{f}=0.5$ [hexane/EtOAc (6:4)]. HPLC analysis Daicel Chiralpak IA, $4.6 \mathrm{~mm} \times 250 \mathrm{~mm}($ hex/IPA $=90: 10,0.5 \mathrm{~mL} / \mathrm{min}, 210 \mathrm{~nm}), \mathrm{t}_{\mathrm{R}}($ minor $)=27.8$ $\min , t_{R}$ (major) $=30.7 \mathrm{~min}, 20 \%$ ee. $[\boldsymbol{\alpha}]_{\mathbf{D}}{ }^{29}=+4.95\left(c=1.0, \mathrm{CHCl}_{3}\right.$ for $\left.20 \% e e\right)$. M. P.: 68$70{ }^{\circ} \mathrm{C} .{ }^{1} \mathbf{H}$ NMR $\left(500 \mathrm{MHz}, \mathrm{CDCl}_{3}\right) \delta$ 7.35-7.24 (m, 5H), $4.67(\mathrm{~s}, 2 \mathrm{H}), 4.32-4.23(\mathrm{~m}, 1 \mathrm{H})$, 2.89-2.85 (m, 1H), 2.79 (dd, $J=18.0,5.0 \mathrm{~Hz}, 1 \mathrm{H}), 2.63(\mathrm{dd}, J=18.0,9.5 \mathrm{~Hz}, 1 \mathrm{H}), 1.84$ (s, $1 \mathrm{H}), 1.49-1.41(\mathrm{~m}, 3 \mathrm{H}), 1.40-1.26(\mathrm{~m}, 5 \mathrm{H}), 0.89(\mathrm{t}, J=7.0 \mathrm{~Hz}, 3 \mathrm{H}) .{ }^{13} \mathbf{C}$ NMR $(125 \mathrm{MHz}$, $\left.\mathrm{CDCl}_{3}\right) \delta 178.9,176.6,135.8,128.6,128.4,127.8,69.6,46.0,42.4,34.9,31.5,28.6,25.4$, 22.5, 13.9. IR (neat) $v 3465,2955,2933,1773,1697,1401,1170,701 \mathrm{~cm}^{-1}$. HRMS (ESI) $\mathrm{m} / \mathrm{z}$ calcd for $\mathrm{C}_{17} \mathrm{H}_{24} \mathrm{NO}_{3}{ }^{+}[\mathrm{M}+\mathrm{H}]^{+} 290.1751$, found 290.1736 .

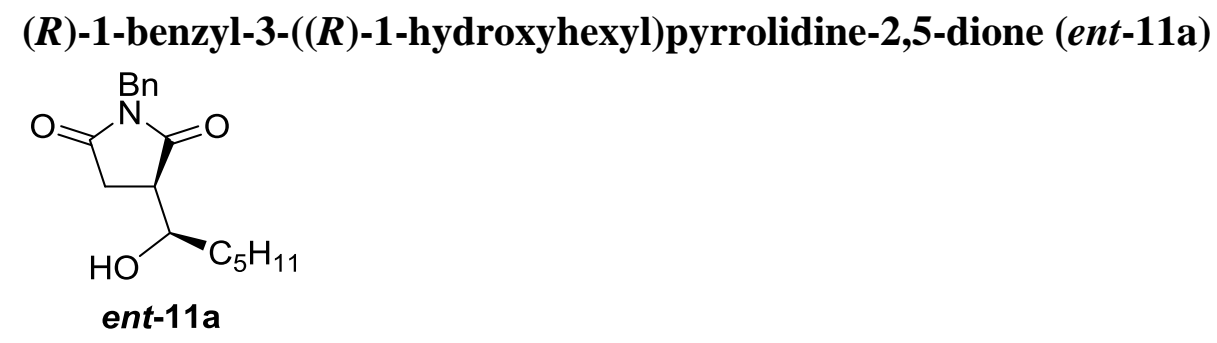


Following the experimental procedure, 3-acylsuccinimide 10a (2.44 g, $8.5 \mathrm{mmol}, 1.0$ equiv) and $\operatorname{RuCl}[(R, R)$-Tsdpen $]$ (p-cymene) (108 $\mathrm{mg}, 0.021 \mathrm{mmol}, 0.02$ equiv) was converted to product ent-11a, which was purified as a white solid (isolated as a single diastereomer, 1.99 $\mathrm{g}, 81 \%$ ) from the crude reaction mixture using flash column chromatography [Silica gel, hexane/EtOAc (85:15)]. HPLC analysis Daicel Chiralcel OD-H, $4.6 \mathrm{~mm} \times 250 \mathrm{~mm}$ (hex/IPA $=90: 10,0.5 \mathrm{~mL} / \mathrm{min}, 210 \mathrm{~nm}), \mathrm{t}_{\mathrm{R}}($ major $)=31.8 \mathrm{~min}, \mathrm{t}_{\mathrm{R}}($ minor $)=35.0 \mathrm{~min}, 95 \%$ ee $\cdot[\boldsymbol{\alpha}]_{\mathrm{D}}{ }^{24}=$ $-8.85\left(c=1.0, \mathrm{CHCl}_{3}\right.$ for $\left.95 \% e e\right)$.

(R)-1-benzyl-3-((R)-1-hydroxydodecyl)pyrrolidine-2,5-dione (ent-11b)

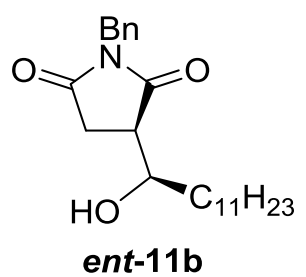

Following the experimental procedure, 3-acylsuccinimide $\mathbf{1 0 b}$ (3.15 g, $8.5 \mathrm{mmol}, 1.0$ equiv) and $\operatorname{RuCl}[(R, R)$-Tsdpen](p-cymene) (108 mg, $0.021 \mathrm{mmol}, 0.02$ equiv) was converted to product $\boldsymbol{e n t - 1 1 b}$, which was purified as a white solid (isolated as a single diastereomer, 2.60 $\mathrm{g}, 82 \%$ ) from the crude reaction mixture using flash column chromatography [Silica gel, hexane/EtOAc (85:15)]. $\mathbf{R}_{f}=0.5$ [hexane/EtOAc (6:4)]. HPLC analysis Daicel Chiralcel OD-H, $4.6 \mathrm{~mm} \times 250 \mathrm{~mm}($ hex/IPA $=94: 6,0.5 \mathrm{~mL} / \mathrm{min}, 210 \mathrm{~nm}), \mathrm{t}_{\mathrm{R}}($ major $)=40.4 \mathrm{~min}, \mathrm{t}_{\mathrm{R}}$ $($ minor $)=46.1 \mathrm{~min}, 97 \%$ ee $[\boldsymbol{\alpha}]_{\mathbf{D}}{ }^{\mathbf{2 6}}=-2.49\left(c=1.0, \mathrm{CHCl}_{3}\right.$ for $\left.97 \% e e\right)$. M. P.: $52-54{ }^{\circ} \mathrm{C} .{ }^{\mathbf{1}} \mathbf{H}$ NMR $\left(500 \mathrm{MHz}, \mathrm{CDCl}_{3}\right) \delta$ 7.36-7.26 (m, 5H), $4.64(\mathrm{~s}, 2 \mathrm{H}), 3.81-3.78(\mathrm{~m}, 1 \mathrm{H}), 3.09(\mathrm{~d}, J=$ $3.0 \mathrm{~Hz}, 1 \mathrm{H}), 2.88-2.75(\mathrm{~m}, 2 \mathrm{H}), 2.49(\mathrm{dd}, J=18.0,5.0 \mathrm{~Hz}, 1 \mathrm{H}), 1.67-1.51(\mathrm{~m}, 4 \mathrm{H}), 1.49$ $1.28(\mathrm{~m}, 16 \mathrm{H}), 0.88(\mathrm{t}, J=6.5 \mathrm{~Hz}, 3 \mathrm{H}) .{ }^{13} \mathbf{C} \mathbf{N M R}\left(125 \mathrm{MHz}, \mathrm{CDCl}_{3}\right) \delta 179.1,175.8,135.6$, 128.7, 128.0, 72.1, 45.1, 42.4, 34.5, 32.0, 31.9, 29.6, 29.6, 29.5, 29.5, 29.3, 25.3, 22.7, 14.1. IR (neat) $v$ 3464, 2923, 2853, 1771, 1694, 1399, 1343, 1170, $696 \mathrm{~cm}^{-1}$. HRMS (ESI) m/z calcd for $\mathrm{C}_{23} \mathrm{H}_{36} \mathrm{NO}_{3}{ }^{+}[\mathrm{M}+\mathrm{H}]^{+} 374.2690$, found 374.2692 .

\section{(S)-1-benzyl-3-((S)-1-hydroxytetradecyl)pyrrolidine-2,5-dione (11c)}

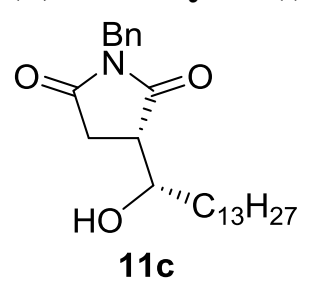

Following the experimental procedure, 3 -acylsuccinimide 10c (3.39 g, $8.5 \mathrm{mmol}, 1.0$ equiv) and $\operatorname{RuCl}[(S, S)$-Tsdpen](p-cymene) (108 $\mathrm{mg}, 0.021 \mathrm{mmol}, 0.02$ equiv) was converted to product 11c, which was purified as a white solid (isolated as a single diastereomer, $2.73 \mathrm{~g}$, $80 \%$ ) from the crude reaction mixture using flash column chromatography [Silica gel, hexane/EtOAc (80:20)]. $\mathbf{R}_{f}=0.5$ [hexane/EtOAc (7:3)]. HPLC analysis Daicel Chiralcel OD-H, $4.6 \mathrm{~mm} \times 250 \mathrm{~mm}($ hex/IPA $=95: 5,0.5 \mathrm{~mL} / \mathrm{min}, 210 \mathrm{~nm}), \mathrm{t}_{\mathrm{R}}($ major $)=45.2 \mathrm{~min}, \mathrm{t}_{\mathrm{R}}$ $($ minor $)=43.0 \mathrm{~min}, 98 \%$ ee $.[\alpha]_{\mathbf{D}}{ }^{\mathbf{2 0}}=+1.6\left(c=0.5, \mathrm{CHCl}_{3}\right.$ for $\left.98 \% e e\right)$. M. P.: $80-86{ }^{\circ} \mathrm{C} .{ }^{1} \mathbf{H}$ NMR $\left(500 \mathrm{MHz}, \mathrm{CDCl}_{3}\right) \delta$ 7.37-7.35 (m, 2H), 7.32-7.26 (m, 3H), $4.65(\mathrm{~s}, 2 \mathrm{H}), 3.81-3.79$ (m, 1H), 3.14 (bs, 1H), 2.87-2.84 (m, 1H), 2.79 (dd, $J=17.5,9.5 \mathrm{~Hz}, 1 \mathrm{H}), 2.50$ (dd, $J=$ 
18.0, $5.0 \mathrm{~Hz}, 1 \mathrm{H}), 1.60-1.49(\mathrm{~m}, 2 \mathrm{H}), 1.37-1.22(\mathrm{~m}, 22 \mathrm{H}), 0.88(\mathrm{t}, J=7.0 \mathrm{~Hz}, 3 \mathrm{H}) .{ }^{13} \mathbf{C}$ NMR $\left(125 \mathrm{MHz}, \mathrm{CDCl}_{3}\right) \delta 179.0,175.9,135.6,128.7,128.6,128.0,72.1,45.1,42.4,34.5$, 32.0, 31.9, 29.7, 29.7, 29.7, 29.6, 29.6, 29.5, 29.5, 29.4, 25.3, 22.7, 14.1. IR (neat) v 3454, 2922, 2852, 1772, 1691, 1432, 1399, 1341, 1170, 1082, 696, $634 \mathrm{~cm}^{-1}$. HRMS (ESI) m/z calcd for $\mathrm{C}_{25} \mathrm{H}_{40} \mathrm{NO}_{3}{ }^{+}[\mathrm{M}+\mathrm{H}]^{+}$402.3003, found 402.2997 .

(R)-1-benzyl-3-((R)-1-hydroxytetradecyl)pyrrolidine-2,5-dione (ent-11c)<smiles>CC(O)C1CC(=O)CC1=O</smiles>

Following the experimental procedure, 3-acylsuccinimide 10c (3.39 g, $8.5 \mathrm{mmol}, 1.0$ equiv) and $\operatorname{RuCl}[(R, R)$-Tsdpen](p-cymene) (108 $\mathrm{mg}, 0.021 \mathrm{mmol}, 0.02$ equiv) was converted to product ent-11c, which was purified as a white solid (isolated as a single diastereomer, 1.99 $\mathrm{g}, 81 \%$ ) from the crude reaction mixture using flash column chromatography [Silica gel, hexane/EtOAc (80:20)]. HPLC analysis Daicel Chiralcel OD-H, $4.6 \mathrm{~mm} \times 250 \mathrm{~mm}$ (hex/IPA $=95: 5,0.5 \mathrm{~mL} / \mathrm{min}, 210 \mathrm{~nm}), \mathrm{t}_{\mathrm{R}}($ major $)=40.1 \mathrm{~min}, \mathrm{t}_{\mathrm{R}}($ minor $)=45.3 \mathrm{~min}, 97 \%$ ee. $[\alpha]_{\mathrm{D}}{ }^{25}=$ $-1.7\left(c=1.0, \mathrm{CHCl}_{3}\right.$ for $\left.97 \% e e\right)$.

\section{Reaction Optimization for the Synthesis of trans-Paraconic Acids 12a ${ }^{a}$}
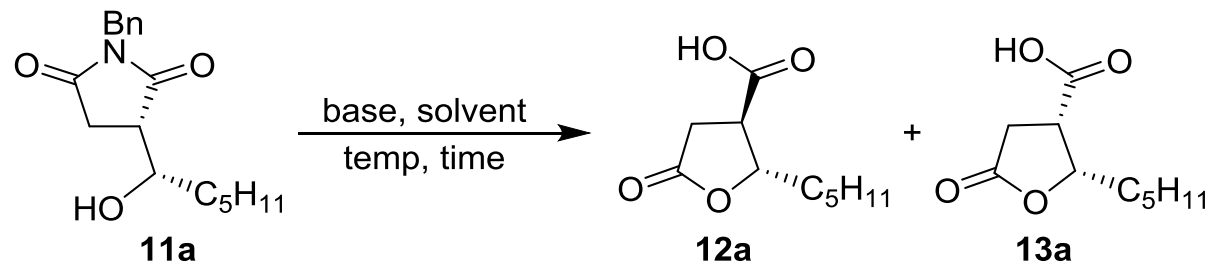

\begin{tabular}{|c|c|c|c|c|c|c|}
\hline entry & $\begin{array}{l}\text { reagent } \quad(\mathrm{mol} \\
\%)\end{array}$ & solvent & temp $\left({ }^{\circ} \mathrm{C}\right)$ & time (h) & $\begin{array}{l}\text { Conversion } \\
(\%)^{b} \\
\mathbf{1 2 a}+\mathbf{1 3 a}\end{array}$ & $\begin{array}{l}\mathrm{dr}^{c} \\
\mathbf{1 2} \mathbf{a}: \mathbf{1 3 a}\end{array}$ \\
\hline 1 & $\mathrm{PTSA}^{d}$ & toluene & reflux & 48 & $-e$ & - \\
\hline 2 & conc. $\mathrm{HCl}$ & - & reflux & 24 & 78 & $1: 2$ \\
\hline 3 & conc. $\mathrm{HCl}$ & 1,4-dioxane & reflux & 96 & 89 & $1: 1$ \\
\hline 4 & $5 \%$ aq. $\mathrm{KOH}$ & 1,4-dioxane & 0 & 18 & 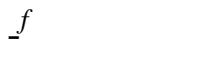 & - \\
\hline 5 & $10 \%$ aq. $\mathrm{KOH}$ & 1,4-dioxane & $\mathrm{rt}$ & 18 & $f$ & - \\
\hline 6 & $10 \%$ aq. $\mathrm{KOH}$ & 1,4-dioxane & reflux & 18 & $74^{f}$ & $1: 1$ \\
\hline 7 & $30 \%$ aq. $\mathrm{KOH}$ & 1,4-dioxane & reflux & 12 & 87 & $2: 1$ \\
\hline 8 & condition $\mathbf{A}^{g}$ & 1,4-dioxane & $55{ }^{\circ} \mathrm{C}$-reflux & 30 & $70^{f}$ & $4: 1$ \\
\hline 9 & condition $\mathbf{B}^{h}$ & 1,4-dioxane & $55^{\circ} \mathrm{C}$-reflux & 24 & 62 & $2: 1$ \\
\hline 10 & condition $\mathbf{C}^{i}$ & 1,4-dioxane & $55{ }^{\circ} \mathrm{C}$-reflux & 28 & $95\left(69^{j}\right)$ & $4: 1$ \\
\hline
\end{tabular}

${ }^{a}$ Reaction conditions: 11a $(0.2 \mathrm{mmol}, 1$ equiv), 1,4-dioxane $(2 \mathrm{~mL})$, regent $(6 \mathrm{~mL})$ unless otherwise mentioned. ${ }^{b}$ Determined by ${ }^{1} \mathrm{H}$ NMR analysis. ${ }^{c}$ Determined by ${ }^{1} \mathrm{H}$ NMR analysis 
of the crude reaction mixture. ${ }^{d}$ PTSA $\left(0.4 \mathrm{mmol}, 2.0\right.$ equiv). ${ }^{e}$ No reaction. ${ }^{f}$ Mixture of hydrolyzed amides were observed. ${ }^{g}$ Condition A: DBU (30 mol\%), after stirring at $55{ }^{\circ} \mathrm{C}$ for $12 \mathrm{~h}, 10 \%$ aq. $\mathrm{KOH}(6 \mathrm{~mL})$ was added, and refluxed for $18 \mathrm{~h} .{ }^{h}$ Condition B: DBU (30 mol\%), after stirring at $55{ }^{\circ} \mathrm{C}$ for $12 \mathrm{~h}, 30 \%$ aq. $\mathrm{KOH}(6 \mathrm{~mL})$ was added, and refluxed for $12 \mathrm{~h}$. ${ }^{i}$ Condition C: DBU (30 mol\%), after stirring at $55{ }^{\circ} \mathrm{C}$ for $12 \mathrm{~h}, 10 \%$ aq. $\mathrm{KOH}(6 \mathrm{~mL})$ was added, refluxed for $4 \mathrm{~h}$, cooled and $\mathrm{KOH}(1.2 \mathrm{~g})$ was added, and refluxed for $12 \mathrm{~h} .{ }^{j}$ Isolated yield of the major diastereomer 12a after column chromatography.

To a solution of alcohols $11 \mathrm{a}(0.2 \mathrm{mmol}, 1.0$ equiv) in 1,4-dioxane ( $2 \mathrm{~mL})$ was added reagent (mentioned in table), stirred at the temperature and time mentioned in table. The reaction mixture was acidified (for the base mediated reactions) with $6 \mathrm{~N} \mathrm{HCl}$ and extracted with dichloromethane ( 3 X $200 \mathrm{~mL}$ ). The organic fraction was dried (sodium sulfate) and concentrated in vacuo to get crude product 12a which was further purified by flash column chromatography.

When alcohol 11a was subjected for cyclization with PTSA in toluene under reflux condition, no reaction was observed. Further alcohol 11 a was heated with conc. $\mathrm{HCl}$ in 1,4dioxane and without 1,4-dioxane under reflux conditions, 12a and 13a were obtained in $89 \%$ $(1: 1 d r)$ and $78 \%(1: 2 d r)$ yields, respectively. After observing poor diastereoselectivity in acid mediated reaction, we moved to base mediated reactions. Initial trials were carried out using $5 \%$ aqueous $\mathrm{KOH}$ at $0{ }^{\circ} \mathrm{C}$ and $10 \%$ aqueous $\mathrm{KOH}$ at $\mathrm{rt}$, but the formation of the desired product was not observed. Then, $10 \%$ aqueous $\mathrm{KOH}$ and 1,4-dioxane under reflux conditions, paraconic acids were obtained in 70\% yield and 1:1 $d r$ along with the mixture of hydrolyzed amides (Table 3, entry 1). Use of $30 \%$ aqueous $\mathrm{KOH}$ resulted in a complete conversion of 11a with higher product yield and 2:1 $d r$ (entry 2). Epimerization of 11a using DBU (30 mol \%) in 1,4-dioxane at $55{ }^{\circ} \mathrm{C}$ followed by an in situ addition of $10 \%$ aqueous $\mathrm{KOH}$ at the reflux temperature produced 12a and 13a in 70\% yield and $4: 1 d r$ along with the mixture of hydrolyzed amides (entry 3). To obtain the higher conversion, after epimerization of alcohol with DBU, $30 \%$ aqueous $\mathrm{KOH}$ was added and gave products in $62 \%$ yield and 2:1 $d r$. After epimerization of alcohol with DBU and refluxing with $10 \%$ aqueous $\mathrm{KOH}$, use of additional $\mathrm{KOH}$ under reflux condition produced desired product 12a and 13a in 95\% yield and $4: 1 d r$.

\section{Experimental procedure for the synthesis of trans-paraconic acids $\mathbf{1 2}$}

A round-bottomed flask equipped with a magnetic stirring bar was charged with alcohol 11 (6.2 mmol, 1.0 equiv) and 1,4-dioxane $(62 \mathrm{~mL})$. The flask was sealed with a rubber septum and DBU $(200 \mu \mathrm{L}, 1.24 \mathrm{mmol}, 0.2$ equiv) was added. The round-bottomed flask was immediately dipped into preheated oil bath $\left(55^{\circ} \mathrm{C}\right)$ and stirred at $55{ }^{\circ} \mathrm{C}$ for $12 \mathrm{~h}$. After cooling down to room temperature, aqueous $\mathrm{KOH}(10 \% \mathrm{w} / \mathrm{v}, 186 \mathrm{~mL})$ was added. The reaction mixture flask was immediately dipped into preheated oil bath at $125{ }^{\circ} \mathrm{C}$ and refluxed for $4 \mathrm{~h}$. The reaction mixture was cooled to room temperature and $\mathrm{KOH}(18.6 \mathrm{~g})$ added. The reaction mixture was refluxed for $12 \mathrm{~h}$, after which it was acidified with $6 \mathrm{~N} \mathrm{HCl}$ and extracted with dichloromethane (3 X $200 \mathrm{~mL}$ ). The organic fraction was dried (sodium sulfate) and concentrated in vacuo to get crude product 12 which was further purified by flash column chromatography. 
(2S,3R)-5-oxo-2-pentyltetrahydrofuran-3-carboxylic acid (12a)

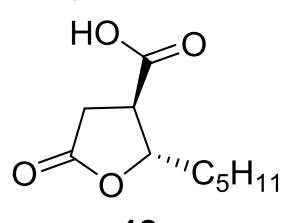

$12 a$

Following the experimental procedure, alcohol 11a (1.79 g, $6.2 \mathrm{mmol}, 1.0$ equiv) was converted to product 12a, which was purified as a white solid (isolated as a single diastereomer, $0.86 \mathrm{~g}, 69 \%$ ) from the crude reaction mixture using flash column chromatography [Silica gel, $\mathrm{Et}_{2} \mathrm{O} /$ hexane/AcOH (40:59:1)]. $\mathbf{R}_{f}=0.4\left[\mathrm{Et}_{2} \mathrm{O} / \mathrm{hexane} / \mathrm{AcOH}\right.$ $(7: 2.8: 0.2)] .[\alpha]_{\mathbf{D}}{ }^{26}=-39.5\left(c=0.5, \mathrm{CHCl}_{3}\right.$ for $\left.96 \% e e\right)$, lit. $^{2}[\alpha]_{\mathrm{D}}{ }^{20}=-54\left(c=0.5, \mathrm{CHCl}_{3}\right)$. M. P.: $102-104{ }^{\circ} \mathrm{C}$, lit. ${ }^{2}$ M. P.: $105-107{ }^{\circ} \mathrm{C} .{ }^{1} \mathbf{H}$ NMR $\left(500 \mathrm{MHz}, \mathrm{CDCl}_{3}\right) \delta$ 4.64-4.60 (m, $1 \mathrm{H}), 3.12-3.07(\mathrm{~m}, 1 \mathrm{H}), 2.94(\mathrm{dd}, J=8.5,17.5 \mathrm{~Hz}, 1 \mathrm{H}), 2.82(\mathrm{dd}, J=18.0,9.5 \mathrm{~Hz}, 1 \mathrm{H})$ 1.84-1.70 (m, 2H), 1.54-1.40 (m, 2H), $1.35-1.30(\mathrm{~m}, 4 \mathrm{H}) 0.90(\mathrm{t}, J=7.0 \mathrm{~Hz}, 3 \mathrm{H}) .{ }^{\mathbf{1 3}} \mathbf{C} \mathbf{N M R}$ $\left(125 \mathrm{MHz}, \mathrm{CDCl}_{3}\right) \delta 175.6,174.3,81.8,45.4,35.3,31.9,31.3,24.8,22.4,13.9$. IR (neat) $v$ 3108, 2929, 2861, 1747, 1723, 1433, 1393, 1240, 1192, $669 \mathrm{~cm}^{-1}$. HRMS (ESI) m/z calcd for $\mathrm{C}_{10} \mathrm{H}_{17} \mathrm{O}_{4}^{+}[\mathrm{M}+\mathrm{H}]^{+}$201.1121, found 201.1100.

\section{$(2 R, 3 S)-5-0 x 0-2-$ undecyltetrahydrofuran-3-carboxylic acid (ent-12b)}

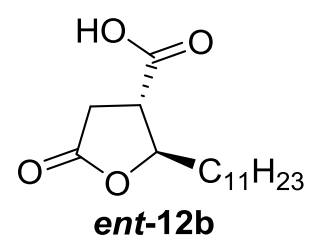

Following the experimental procedure, alcohol ent-11b $(2.31 \mathrm{~g}, 6.2 \mathrm{mmol}, 1.0$ equiv) was converted to product ent-12b, which was purified as a white solid (isolated as a single diastereomer, $1.23 \mathrm{~g}, 70 \%$ ) from the crude reaction mixture using flash column chromatography [Silica gel, $\mathrm{Et}_{2} \mathrm{O} /$ hexane/AcOH (40:59:1)]. $\mathbf{R}_{f}=0.5\left[\mathrm{Et}_{2} \mathrm{O} / \mathrm{hexane} / \mathrm{AcOH}\right.$ $(7: 2.8: 0.2)] \cdot[\alpha]_{\mathbf{D}}{ }^{28}=+46.7\left(c=0.33, \mathrm{CHCl}_{3}\right.$ for $\left.96 \% e e\right)$, lit. $^{3}[\alpha]_{\mathrm{D}}{ }^{20}=+44.8(c=0.25$, $\left.\mathrm{CHCl}_{3}\right)$. M. P.: $112-114{ }^{\circ} \mathrm{C}$, lit. ${ }^{4}$ M. P.: $116-118{ }^{\circ} \mathrm{C} .{ }^{\mathbf{1}} \mathbf{H}$ NMR $\left(500 \mathrm{MHz}, \mathrm{CDCl}_{3}\right) \delta 4.64-$ $4.60(\mathrm{~m}, 1 \mathrm{H}), 3.11-3.06(\mathrm{~m}, 1 \mathrm{H}), 2.94(\mathrm{dd}, J=17.5,8.0 \mathrm{~Hz}, 1 \mathrm{H}), 2.82(\mathrm{dd}, J=18.0,10.0 \mathrm{~Hz}$, $1 \mathrm{H}), 1.83-1.70(\mathrm{~m}, 2 \mathrm{H}), 1.53-1.47(\mathrm{~m}, 1 \mathrm{H}), 1.45-1.37(\mathrm{~m}, 1 \mathrm{H}), 1.35-1.26(\mathrm{~m}, 16 \mathrm{H}), 0.88(\mathrm{t}$, $J=6.5 \mathrm{~Hz}, 3 \mathrm{H}) .{ }^{13} \mathbf{C}$ NMR $\left(125 \mathrm{MHz}, \mathrm{CDCl}_{3}\right) \delta 175.9,174.4,81.8,45.4,35.4,31.9,29.6$, 29.5, 29.4, 29.3, 29.2, 25.2, 22.7, 14.1. IR (neat) $v$ 3099, 2953, 2921, 2849, 1748, 1720, 1463, 1238, 1192, $669 \mathrm{~cm}^{-1}$. HRMS (ESI) m/z calcd for $\mathrm{C}_{16} \mathrm{H}_{29} \mathrm{O}_{4}{ }^{+}[\mathrm{M}+\mathrm{H}]^{+}$285.2060, found 285.2084 .

(2R,3S)-5-oxo-2-tridecyltetrahydrofuran-3-carboxylic acid (ent-12c)<smiles>CC1OC(=O)C[C@@H]1C(=O)O</smiles> 
Following the experimental procedure, alcohol ent-11c ( $2.49 \mathrm{~g}, 6.2 \mathrm{mmol}, 1.0$ equiv) was converted to product ent-12c, which was purified as a white solid (isolated as a single diastereomer, $1.18 \mathrm{~g}, 61 \%$ ) from the crude reaction mixture using flash column chromatography [Silica gel, $\mathrm{Et}_{2} \mathrm{O} / \mathrm{hexane} / \mathrm{AcOH}$ (50:50:1)] to yield the title compound as a white solid: $\mathbf{R}_{f}=0.5\left[\mathrm{Et}_{2} \mathrm{O} / \mathrm{hexane} / \mathrm{AcOH}(7: 3: 0.1)\right] .[\boldsymbol{\alpha}]_{\mathbf{D}}{ }^{25}=+36.8\left(c=0.5, \mathrm{CHCl}_{3}\right.$ for $96 \%$ $e e)$, lit. ${ }^{4}[\alpha]_{\mathrm{D}}{ }^{25}+38.8\left(c=0.35, \mathrm{CHCl}_{3}\right.$ for $93 \%$ ee $)$. M. P.: $103-105{ }^{\circ} \mathrm{C}$, lit. ${ }^{4}$ M. P.: $104-106$ ${ }^{\circ} \mathrm{C} .{ }^{1} \mathbf{H}$ NMR $\left(500 \mathrm{MHz}, \mathrm{CDCl}_{3}\right) \delta 4.64-4.60(\mathrm{~m}, 1 \mathrm{H}), 3.12-3.07(\mathrm{~m}, 1 \mathrm{H}), 2.94(\mathrm{dd}, J=18.0$, $8.5 \mathrm{~Hz}, 1 \mathrm{H}), 2.82(\mathrm{dd}, J=18.0,9.5 \mathrm{~Hz}, 1 \mathrm{H}), 1.83-1.70(\mathrm{~m}, 2 \mathrm{H}), 1.54-1.47(\mathrm{~m}, 1 \mathrm{H}), 1.45-$ $1.38(\mathrm{~m}, 1 \mathrm{H}), 1.37-1.22(\mathrm{~m}, 20 \mathrm{H}), 0.88(\mathrm{t}, J=7.0 \mathrm{~Hz}, 3 \mathrm{H}) .{ }^{13} \mathbf{C} \mathbf{N M R}\left(125 \mathrm{MHz}, \mathrm{CDCl}_{3}\right) \delta$ 176.1, 174.4, 81.8, 45.4, 35.4, 31.9, 31.9, 29.7, 29.6, 29.6, 29.5, 29.4, 29.4, 29.2, 25.2, 22.7, 14.1. IR (neat) $v$ 3106, 2920, 2849, 1747, 1720, 1463, 1434, 1236, 1199, 1191, 951, 670 $\mathrm{cm}^{-1}$. HRMS (ESI) $\mathrm{m} / \mathrm{z}$ calcd for $\mathrm{C}_{18} \mathrm{H}_{33} \mathrm{O}_{4}{ }^{+}[\mathrm{M}+\mathrm{H}]^{+}$313.2373, found 313.2367.

\section{Experimental procedure for the synthesis of cis-paraconic acids $\mathbf{1 3}$}

To a solution of alcohol 11 (3.1 mmol, 1.0 equiv) in 1,4-dioxane (31 mL), 5\% aq. KOH (31 $\mathrm{mL}$ ) were added at $0{ }^{\circ} \mathrm{C}$ and stirred at this temperature for $18 \mathrm{~h}$. After complete conversion of reactant (monitored by ${ }^{1} \mathrm{H} \mathrm{NMR}$ ), the reaction mixture was acidified with $2 \mathrm{M} \mathrm{HCl}$ and extracted with dichloromethane $(3 \times 50 \mathrm{~mL})$. The combined organic fractions were dried (sodium sulfate) and concentrated in vacuo to get crude product. The crude product was used directly for the next step.

The crude product was dissolved in a mixture of $\mathrm{Ac}_{2} \mathrm{O}(124 \mathrm{~mL})$ and $\mathrm{AcOH}(62 \mathrm{~mL})$ at $\mathrm{rt}$, and allowed to stir for $10 \mathrm{~min} . \mathrm{NaNO}_{2}(4.28 \mathrm{~g}, 62.0 \mathrm{mmol}, 20.0$ equiv) was added and after $15 \mathrm{~h}$ stirring at $\mathrm{rt}$, liquid was removed in vacuo to afford white solid. White solid was dissolved in $10 \%$ aq. $\mathrm{KOH}(250 \mathrm{~mL}, \mathrm{pH}>10)$, and 1,4-dioxane $(250 \mathrm{~mL})$ was added at $0{ }^{\circ} \mathrm{C}$. The mixture was stirred at this temperature for $3 \mathrm{~h}$, acidified with conc. $\mathrm{HCl}(250 \mathrm{~mL})$ and 1,4-dioxane $(250 \mathrm{~mL})$ was added. The reaction mixture allowed to stir at $\mathrm{rt}$ for $3 \mathrm{~h}$ and extracted with DCM (250 mL X 3), organic fraction was dried (sodium Sulfate) and concentrated in vacuo to get crude product $\mathbf{1 3}$ which was further purified by flash column chromatography.

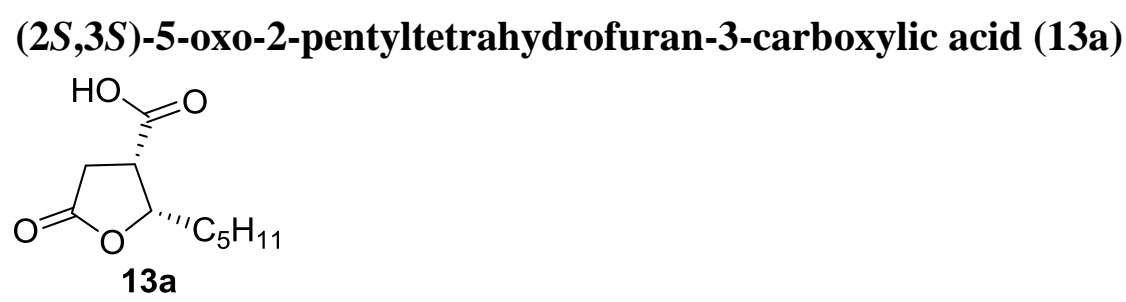

Following the experimental procedure, alcohol 11a $(0.90 \mathrm{~g}, 3.1 \mathrm{mmol}, 1.0$ equiv) was converted to product 13a which was purified as a white solid (isolated as a single diastereomer, $496 \mathrm{mg}, 80 \%$ ) from the crude reaction mixture using flash column chromatography [Silica gel, $\mathrm{Et}_{2} \mathrm{O} /$ hexane/AcOH (45:54:1)]. $\mathbf{R}_{f}=0.4\left[\mathrm{Et}_{2} \mathrm{O} / \mathrm{hexane} / \mathrm{AcOH}\right.$ $(7: 2.8: 0.2)] .[\alpha]_{\mathbf{D}}{ }^{23}=-70.8\left(c=0.2, \mathrm{CHCl}_{3}\right.$ for $\left.94 \% e e\right)$, lit. $^{5}[\alpha]_{\mathrm{D}}{ }^{25}=-44.1\left(c=0.16, \mathrm{CHCl}_{3}\right.$ for $97 \%$ ee). M. P.: $104-106{ }^{\circ} \mathrm{C}$, lit. ${ }^{5}$ M. P.: $102-104{ }^{\circ} \mathrm{C} .{ }^{\mathbf{1}} \mathbf{H}$ NMR $\left(500 \mathrm{MHz}, \mathrm{CDCl}_{3}\right) \delta$ $4.69-4.64(\mathrm{~m}, 1 \mathrm{H}), 3.49-3.45(\mathrm{~m}, 1 \mathrm{H}), 2.90(\mathrm{dd}, J=17.5,5.0 \mathrm{~Hz}, 1 \mathrm{H}), 2.70(\mathrm{dd}, J=17.5,8.5$ $\mathrm{Hz}, 1 \mathrm{H}), 1.69-1.61(\mathrm{~m}, 1 \mathrm{H}), 1.58-1.53(\mathrm{~m}, 1 \mathrm{H}), 1.48-1.39$ (m, 1H), 1.36-1.26 (m, 4H), 0.89 
$(\mathrm{t}, J=7.0 \mathrm{~Hz}, 3 \mathrm{H}) .{ }^{13} \mathbf{C}$ NMR $\left(125 \mathrm{MHz}, \mathrm{CDCl}_{3}\right) \delta 175.6,174.8,80.3,44.2,31.9,31.3,31.3$, 25.5, 22.4, 13.9. IR (neat) $v$ 3126, 2955, 2929, 2860, 1737, 1432, 1356, 1238, 1180, 669 $\mathrm{cm}^{-1}$. HRMS (ESI) $\mathrm{m} / \mathrm{z}$ calcd for $\mathrm{C}_{10} \mathrm{H}_{17} \mathrm{O}_{4}{ }^{+}[\mathrm{M}+\mathrm{H}]^{+}$201.1121, found 201.1139.

\section{(2R,3R)-5-0xo-2-pentyltetrahydrofuran-3-carboxylic acid (ent-13a)}

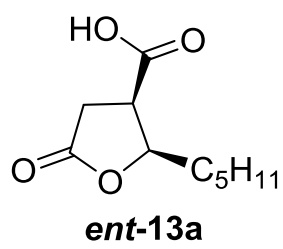

Following the experimental procedure, alcohol ent-11a $(0.90 \mathrm{~g}, 3.1 \mathrm{mmol}, 1.0$ equiv) was converted to product ent-13a which was purified as a white solid (isolated as a single diastereomer, $0.50 \mathrm{~g}, 81 \%$ ) from the crude reaction mixture using flash column chromatography [Silica gel, $\mathrm{Et}_{2} \mathrm{O} /$ hexane/AcOH $\left.(45: 54: 1)\right] .[\alpha]_{\mathbf{D}}{ }^{23}=+68.9\left(c=0.2, \mathrm{CHCl}_{3}\right.$ for $95 \%$ ee $)$, lit. ${ }^{4}[\alpha]_{\mathrm{D}}{ }^{25}+44.0\left(c=0.14, \mathrm{CHCl}_{3}\right.$ for $\left.94 \% e e\right)$.

\section{(2S,3S)-5-oxo-2-tridecyltetrahydrofuran-3-carboxylic acid (13c)

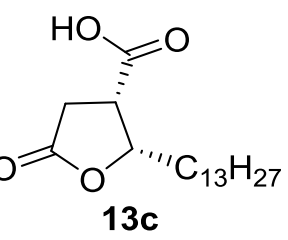

Following the experimental procedure, alcohol 11c (1.24 g, $3.1 \mathrm{mmol}, 1.0$ equiv) was converted to product 13c, which was purified as a white solid (isolated as a single diastereomer, $0.80 \mathrm{~g}, 82 \%$ ) from the crude reaction mixture using flash column chromatography [Silica gel, $\mathrm{Et}_{2} \mathrm{O} /$ hexane/AcOH (50:50:1)]. $\mathbf{R}_{f}=0.4\left[\mathrm{Et}_{2} \mathrm{O} / \mathrm{hexane} / \mathrm{AcOH}\right.$ (7:3:0.1)]. $[\boldsymbol{\alpha}]_{\mathbf{D}}{ }^{\mathbf{2 8}}=-81.25\left(c=0.2, \mathrm{CHCl}_{3}\right.$ for $\left.95 \% e e\right)$, M. P.: $113-115^{\circ} \mathrm{C} .{ }^{\mathbf{1}} \mathbf{H}$ NMR $(500$ $\left.\mathrm{MHz}, \mathrm{CDCl}_{3}\right) \delta 4.68-4.64(\mathrm{~m}, 1 \mathrm{H}), 3.49-3.44(\mathrm{~m}, 1 \mathrm{H}), 2.90(\mathrm{dd}, J=18.0,5.5 \mathrm{~Hz}, 1 \mathrm{H}), 2.70$ $(\mathrm{dd}, J=18.0,8.5 \mathrm{~Hz}, 1 \mathrm{H}), 1.71-1.63(\mathrm{~m}, 2 \mathrm{H}), 1.57-1.51(\mathrm{~m}, 1 \mathrm{H}), 1.47-1.38(\mathrm{~m}, 1 \mathrm{H}), 1.37-$ $1.26(\mathrm{~m}, 20 \mathrm{H}), 0.88(\mathrm{t}, J=7.0 \mathrm{~Hz}, 3 \mathrm{H}) .{ }^{13} \mathbf{C ~ N M R}\left(125 \mathrm{MHz}, \mathrm{CDCl}_{3}\right) \delta 175.5,174.8,80.3$, $44.2,31.9,31.9,31.3,29.7,29.7,29.7,29.6,29.5,29.4,29.4,29.2,25.9,22.7,14.1$. IR (neat) $v$ 3052, 2917, 2850, 1752, 1738, 1470, 1422, 1360, 1202, 1186, 1049, 1010, $668 \mathrm{~cm}^{-1}$. HRMS (ESI) $\mathrm{m} / \mathrm{z}$ calcd for $\mathrm{C}_{18} \mathrm{H}_{33} \mathrm{O}_{4}{ }^{+}[\mathrm{M}+\mathrm{H}]^{+}$313.2373, found 313.2350.

\section{Experimental procedure for the synthesis of paraconic esters 16 or 17}

A round-bottomed flask equipped with a magnetic stirring bar was charged with paraconic acid 12 or 13 ( $0.2 \mathrm{mmol}, 1.0$ equiv) and $\mathrm{Cs}_{2} \mathrm{CO}_{3}(65 \mathrm{mg}, 0.2 \mathrm{mmol}, 1.0$ equiv). The flask was capped with a rubber septum, purged with nitrogen, DMF $(1 \mathrm{~mL})$ and benzyl bromide (28 $\mu \mathrm{L}, 0.24 \mathrm{mmol}, 1.2$ equiv) was added. Reaction mixture was allowed stir for $18 \mathrm{~h}$ at room temperature, after which it was quenched with $1 \mathrm{M} \mathrm{HCl}(5 \mathrm{~mL})$ and diluted with water. The mixture was extracted with $\mathrm{Et}_{2} \mathrm{O}(15 \mathrm{~mL})$, organic fractions was dried (sodium sulfate) and concentrated in vacuo to get crude benzyl ester which was further purified by flash column chromatography. 


\section{benzyl (2S,3R)-5-oxo-2-pentyltetrahydrofuran-3-carboxylate (16a)}<smiles>O=C1C[C@H](C(=O)O)[C@@H]([18OH])[C@@H]1[18OH]</smiles>

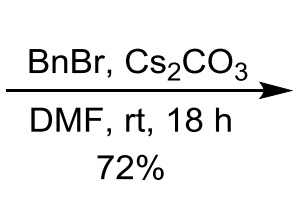

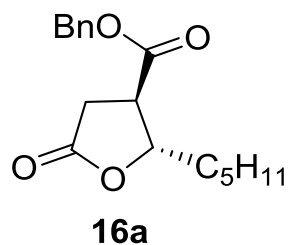

Following the experimental procedure, paraconic acid 12a (40 mg, $0.2 \mathrm{mmol}, 1.0$ equiv) was converted to product 16a which was purified as a colorless liquid (42 $\mathrm{mg}, 72 \%$ ) from the crude reaction mixture using flash column chromatography [Silica gel, hexane/EtOAc (90:10)]. $\mathbf{R}_{f}=0.6$ [hexane/EtOAc (7:3)]. HPLC analysis Daicel Chiralpak AD-H, $4.6 \mathrm{~mm} \times$ $250 \mathrm{~mm}($ hex $/ \mathrm{IPA}=88: 12,0.5 \mathrm{~mL} / \mathrm{min}, 210 \mathrm{~nm}), \mathrm{t}_{\mathrm{R}}($ minor $)=16.4 \mathrm{~min}, \mathrm{t}_{\mathrm{R}}($ major $)=18.2$ $\left.\min , 96 \% e e \cdot{ }_{[\alpha}\right]_{\mathbf{D}}{ }^{\mathbf{2 8}}=-23.8\left(c=0.6, \mathrm{CHCl}_{3}\right.$ for $\left.96 \% e e\right) .{ }^{\mathbf{1}} \mathbf{H} \mathbf{~ N M R}\left(500 \mathrm{MHz}, \mathrm{CDCl}_{3}\right) \delta$ $7.39-7.26(\mathrm{~m}, 5 \mathrm{H}), 5.20(\mathrm{~d}, J=12.0 \mathrm{~Hz}, 1 \mathrm{H}), 5.16(\mathrm{~d}, J=12.5 \mathrm{~Hz}, 1 \mathrm{H}), 4.58-4.54(\mathrm{~m}, 1 \mathrm{H})$, 3.09-3.04 (m, 1H), 2.93 (dd, $J=17.5,9.0 \mathrm{~Hz}$ ), 2.78 (dd, $J=18.0,10.0 \mathrm{~Hz}, 1 \mathrm{H}), 1.76-1.70$ $(\mathrm{m}, 2 \mathrm{H}), 1.42-1.28(\mathrm{~m}, 2 \mathrm{H}), 1.28-1.27(\mathrm{~m}, 4 \mathrm{H}), 0.88(\mathrm{t}, J=7.0 \mathrm{~Hz}, 3 \mathrm{H}) .{ }^{13} \mathrm{C}$ NMR $(125$ $\left.\mathrm{MHz}, \mathrm{CDCl}_{3}\right) \delta 174.3,170.9,135.1,128.8,128.7,128.4,81.9,67.5,45.8,35.3,32.2,31.3$, 24.8, 22.4, 13.9. IR (neat) $v$ 2923, 2851, 1781, 1735, 1456, 1260, 1172, $698 \mathrm{~cm}^{-1}$. HRMS (ESI) $\mathrm{m} / \mathrm{z}$ calcd for $\mathrm{C}_{17} \mathrm{H}_{23} \mathrm{O}_{4}{ }^{+}[\mathrm{M}+\mathrm{H}]^{+} 291.1591$, found 291.1563 .

\section{benzyl $(2 R, 3 S)-5$-oxo-2-undecyltetrahydrofuran-3-carboxylate (ent-16b)}

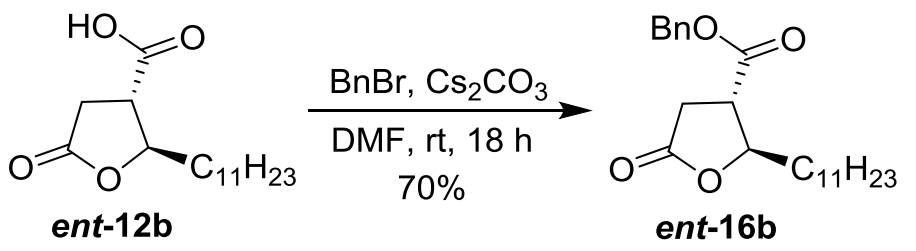

Following the experimental procedure, paraconic acid ent-12b (57 mg, $0.2 \mathrm{mmol}, 1.0$ equiv) was converted to product ent-16b which was purified as a colorless liquid (52 $\mathrm{mg}, 70 \%$ ) from the crude reaction mixture using flash column chromatography [Silica gel, hexane/EtOAc (90:10)]. $\mathbf{R}_{f}=0.6$ [hexane/EtOAc (7:3)]. HPLC analysis Daicel Chiralpak AD-H, $4.6 \mathrm{~mm} \times$ $250 \mathrm{~mm}($ hex $/ \mathrm{IPA}=90: 10,0.5 \mathrm{~mL} / \mathrm{min}, 210 \mathrm{~nm}), \mathrm{t}_{R}($ major $)=13.4 \mathrm{~min}, \mathrm{t}_{R}($ minor $)=14.4$ $\min , 96 \% e e .[\boldsymbol{\alpha}]_{\mathbf{D}}{ }^{\mathbf{2 8}}=+36.4\left(c=0.4, \mathrm{CHCl}_{3}\right.$ for $\left.96 \% e e\right) .{ }^{1} \mathbf{H} \mathbf{~ N M R}\left(500 \mathrm{MHz}, \mathrm{CDCl}_{3}\right) \delta$ 7.40-7.33 (m, 5H), $5.20(\mathrm{~d}, J=12.0 \mathrm{~Hz}, 1 \mathrm{H}), 5.16(\mathrm{~d}, J=12.5 \mathrm{~Hz}, 1 \mathrm{H}), 4.58-4.54(\mathrm{~m}, 1 \mathrm{H})$, 3.09-3.04 (m, 1H), $2.92(\mathrm{dd}, J=18.0,9.0 \mathrm{~Hz}, 1 \mathrm{H}), 2.77(\mathrm{dd}, J=18.0,9.5 \mathrm{~Hz}, 1 \mathrm{H}), 1.76-1.70$ $(\mathrm{m}, 2 \mathrm{H}), 1.47-1.43(\mathrm{~m}, 2 \mathrm{H}), 1.39-1.26(\mathrm{~m}, 16 \mathrm{H}), 0.89(\mathrm{t}, J=7.0 \mathrm{~Hz}, 3 \mathrm{H}) .{ }^{13} \mathbf{C}$ NMR $(125$ $\left.\mathrm{MHz}, \mathrm{CDCl}_{3}\right) \delta 174.3,171.0,135.1,128.8,128.7,128.4,81.9,67.5,45.8,35.3,32.2,31.9$, 29.6, 29.5, 29.4, 29.3, 29.2, 25.1, 22.7, 14.1. IR (neat) $v$ 2924, 2854, 1783, 1738, 1456, 1193 , 1002, $697 \mathrm{~cm}^{-1}$. HRMS (ESI) $\mathrm{m} / \mathrm{z}$ calcd for $\mathrm{C}_{23} \mathrm{H}_{35} \mathrm{O}_{4}{ }^{+}[\mathrm{M}+\mathrm{H}]^{+} 375.2530$, found 375.2521 .

\section{(2R,3S)-benzyl 5-oxo-2-tridecyltetrahydrofuran-3-carboxylate (ent-16c)}

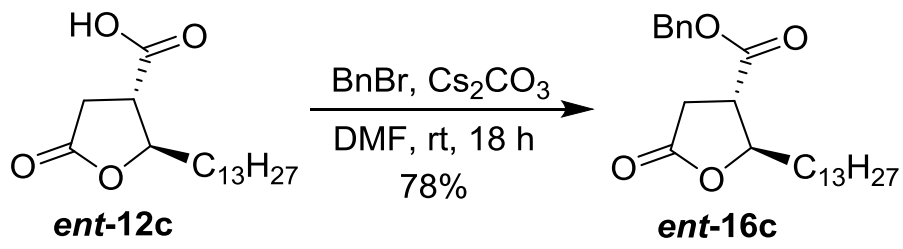


Following the experimental procedure, paraconic acid ent-12c (62 mg, $0.2 \mathrm{mmol}, 1.0$ equiv) was converted to product ent-16c which was purified as a colorless liquid (63 $\mathrm{mg}, 78 \%$ ) from the crude reaction mixture using flash column chromatography [Silica gel, hexane/EtOAc (80:20)]. $\mathbf{R}_{f}=0.5$ [hexane/EtOAc (7:3)]. HPLC analysis Daicel Chiralpak AD-H, $4.6 \mathrm{~mm} \times$ $250 \mathrm{~mm}($ hex $/ \mathrm{IPA}=95: 5,0.5 \mathrm{~mL} / \mathrm{min}, 210 \mathrm{~nm}), \mathrm{t}_{R}($ major $)=14.8 \mathrm{~min}, \mathrm{t}_{\mathrm{R}}($ minor $)=15.8$ $\min , 96 \%$ ee. $[\boldsymbol{\alpha}]_{\mathbf{D}}{ }^{\mathbf{2 4}}=+44.3\left(c=0.25, \mathrm{CHCl}_{3}\right.$ for $96 \%$ ee $) .{ }^{\mathbf{1}} \mathbf{H} \mathbf{~ N M R}\left(500 \mathrm{MHz}, \mathrm{CDCl}_{3}\right) \delta$ $7.40-7.33(\mathrm{~m}, 5 \mathrm{H}), 5.20(\mathrm{~d}, J=12.0 \mathrm{~Hz}, 1 \mathrm{H}), 5.16(\mathrm{~d}, J=12.0 \mathrm{~Hz}, 1 \mathrm{H}), 4.58-4.54(\mathrm{~m}, 1 \mathrm{H})$, 3.09-3.04 (m, 1H), 2.92 (dd, $J=18.0,9.0 \mathrm{~Hz}, 1 \mathrm{H}), 2.77$ (dd, $J=18.0,9.5 \mathrm{~Hz}, 1 \mathrm{H}), 1.78-$ $1.67(\mathrm{~m}, 2 \mathrm{H}), 1.50-1.41(\mathrm{~m}, 1 \mathrm{H}), 1.39-1.33(\mathrm{~m}, 1 \mathrm{H}), 1.32-1.23(\mathrm{~m}, 20 \mathrm{H}), 0.88(\mathrm{t}, J=7.0 \mathrm{~Hz}$, $3 \mathrm{H}) .{ }^{13} \mathrm{C}$ NMR $\left(125 \mathrm{MHz}, \mathrm{CDCl}_{3}\right) \delta 174.3,171.0,135.1,128.8,128.7,128.4,81.9,67.5$, $45.8,35.3,32.2,31.9,29.7,29.7,29.6,29.5,29.4,29.4,29.2,25.1,22.7,14.1$. IR (neat) $v$ 2923, 2853, 1784, 1738, 1457, 1260, 1193, 1002, 751, $698 \mathrm{~cm}^{-1}$. HRMS (ESI) m/z calcd for $\mathrm{C}_{25} \mathrm{H}_{39} \mathrm{O}_{4}^{+}[\mathrm{M}+\mathrm{H}]^{+}$403.2843, found 403.2836.

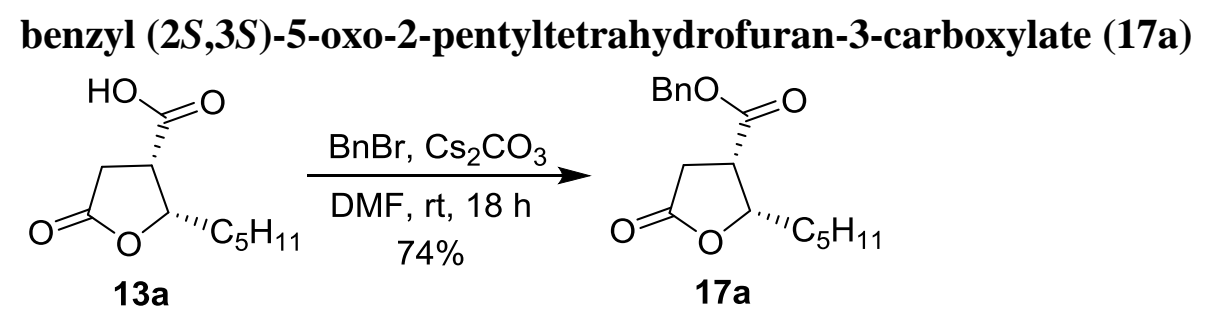

Following the experimental procedure, paraconic acid 13a (40 mg, $0.2 \mathrm{mmol}, 1.0$ equiv) was converted to product $\mathbf{1 7} \mathbf{a}$ which was purified as a white solid (43 $\mathrm{mg}, 74 \%$ ) from the crude reaction mixture using flash column chromatography [Silica gel, hexane/EtOAc (80:20)]. $\mathbf{R}_{\boldsymbol{f}}$ $=0.5[$ hexane/EtOAc (7:3)]. HPLC analysis Daicel Chiralpak AD-H, $4.6 \mathrm{~mm} \times 250 \mathrm{~mm}$ $($ hex $/ \mathrm{IPA}=88: 12,0.5 \mathrm{~mL} / \mathrm{min}, 210 \mathrm{~nm}), \mathrm{t}_{\mathrm{R}}($ major $)=18.2 \mathrm{~min}, \mathrm{t}_{\mathrm{R}}($ minor $)=22.4 \mathrm{~min}, 94 \%$ $e e .[\alpha]_{\mathbf{D}}{ }^{24}=-57.4\left(c=0.25, \mathrm{CHCl}_{3}\right.$ for $\left.94 \% e e\right)$. M. P.: $74-76{ }^{\circ} \mathrm{C} .{ }^{\mathbf{1}} \mathbf{H}$ NMR $(500 \mathrm{MHz}$, $\left.\mathrm{CDCl}_{3}\right) \delta 7.38-7.34(\mathrm{~m}, 5 \mathrm{H}), 5.21(\mathrm{~d}, J=12.0 \mathrm{~Hz}, 1 \mathrm{H}), 5.14(\mathrm{~d}, J=12.0 \mathrm{~Hz}, 1 \mathrm{H}), 4.62-4.58$ (m, 1H), 3.47-3.43 (m, 1H), $2.90(\mathrm{dd}, J=17.5,5.5 \mathrm{~Hz}, 1 \mathrm{H}), 2.66(\mathrm{dd}, J=17.5,8.5 \mathrm{~Hz}, 1 \mathrm{H})$, 1.48-1.40 (m, 3H), 1.37-1.27 (m, 1H), 1.25-1.14 (m, 4H), $0.85(\mathrm{t}, J=7 \mathrm{~Hz}, 3 \mathrm{H}) .{ }^{13} \mathbf{C}$ NMR $\left(125 \mathrm{MHz}, \mathrm{CDCl}_{3}\right) \delta 174.8,170.2,135.0,128.8,128.7,128.7,80.6,67.2,44.3,31.8,31.3$, 31.2, 25.4, 22.3, 13.9. IR (neat) $v$ 2953, 2924, 2847, 1781, 1735, 1457, 1168, $698 \mathrm{~cm}^{-1}$. HRMS (ESI) m/z calcd for $\mathrm{C}_{17} \mathrm{H}_{23} \mathrm{O}_{4}{ }^{+}[\mathrm{M}+\mathrm{H}]^{+}$291.1591, found 291.1570.

\section{benzyl (2R,3R)-5-oxo-2-pentyltetrahydrofuran-3-carboxylate (ent-17a)}

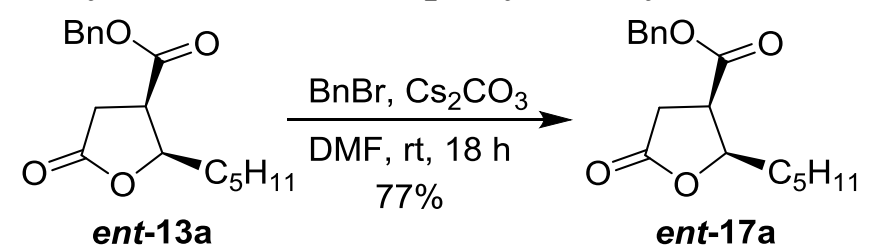

Following the experimental procedure, paraconic acid ent-13a (40 mg, $0.2 \mathrm{mmol}, 1.0$ equiv) was converted to product ent-17a which was purified as a white solid (45 $\mathrm{mg}, 77 \%$ ) from the crude reaction mixture using flash column chromatography [Silica gel, hexane/EtOAc (80:20)]. HPLC analysis Daicel Chiralpak AD-H, $4.6 \mathrm{~mm} \times 250 \mathrm{~mm}($ hex/IPA = 92:8, 0.5 
$\mathrm{mL} / \min , 210 \mathrm{~nm}), \mathrm{t}_{\mathrm{R}}($ minor $)=18.4 \mathrm{~min}, \mathrm{t}_{\mathrm{R}}($ major $)=22.8 \mathrm{~min}, 95 \%$ ee. $[\boldsymbol{\alpha}]_{\mathrm{D}}{ }^{24}=+65.6(c=$ $0.25, \mathrm{CHCl}_{3}$ for $95 \% \mathrm{ee}$ ).

\section{(2S,3S)-benzyl 5-oxo-2-tridecyltetrahydrofuran-3-carboxylate (17c)}
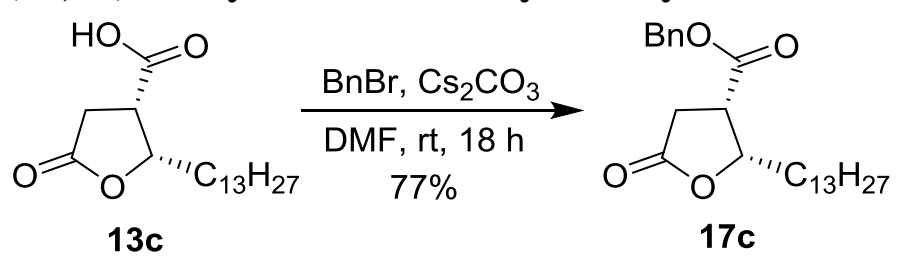

Following the experimental procedure, paraconic acid 13c (62 mg, $0.2 \mathrm{mmol}, 1.0$ equiv) was converted to product 17c which was purified as a white solid (62 mg, 77\%) from the crude reaction mixture using flash column chromatography [Silica gel, hexane/EtOAc (80:20)]. $\mathbf{R}_{\boldsymbol{f}}$ $=0.5$ [hexane/EtOAc (7:3)]. HPLC analysis Daicel Chiralpak AD-H, $4.6 \mathrm{~mm} \times 250 \mathrm{~mm}$ $($ hex $/ \mathrm{IPA}=90: 10,0.5 \mathrm{~mL} / \mathrm{min}, 210 \mathrm{~nm}), \mathrm{t}_{R}($ major $)=12.3 \mathrm{~min}, \mathrm{t}_{\mathrm{R}}($ minor $)=14.1 \mathrm{~min}, 95 \%$ $e e .[\boldsymbol{\alpha}]_{\mathbf{D}}{ }^{29}=-98.3\left(c=0.5, \mathrm{CHCl}_{3}\right.$ for $\left.95 \% e e\right)$. M. P.: $87-88{ }^{\circ} \mathrm{C} .{ }^{\mathbf{1}} \mathbf{H}$ NMR $(500 \mathrm{MHz}$, $\left.\mathrm{CDCl}_{3}\right) \delta 7.41-7.33(\mathrm{~m}, 5 \mathrm{H}), 5.21(\mathrm{~d}, J=12.0 \mathrm{~Hz}, 1 \mathrm{H}), 5.15(\mathrm{~d}, J=12.0 \mathrm{~Hz}, 1 \mathrm{H}), 4.62-4.58$ (m, 1H), 3.50-3.43 (m, 1H), 2.91 (dd, $J=17.5,5.5 \mathrm{~Hz}, 1 \mathrm{H}), 2.66(\mathrm{dd}, J=17.5,8.5 \mathrm{~Hz}, 1 \mathrm{H})$, 1.51-1.37 (m, 2H), 1.33-1.19 (m, 22H), $0.88(\mathrm{t}, J=7.0 \mathrm{~Hz}, 3 \mathrm{H}) .{ }^{13} \mathbf{C}$ NMR $(125 \mathrm{MHz}$, $\left.\mathrm{CDCl}_{3}\right) \delta 174.8,170.2,135.0,128.8,128.7,128.7,80.5,67.2,44.3,31.9,31.8,31.3,29.7$, 29.7, 29.6, 29.5, 29.4, 29.3, 29.2, 25.8, 22.7, 14.1. IR (neat) $v 2919,2850,1771,1720,1470$, 1399, 1234, 1168, 990, 893, 757, $700 \mathrm{~cm}^{-1}$. HRMS (ESI) m/z calcd for $\mathrm{C}_{25} \mathrm{H}_{39} \mathrm{O}_{4}{ }^{+}[\mathrm{M}+\mathrm{H}]^{+}$ 403.2843, found 403.2841.

\section{Experimental procedure for $\alpha$-methylenation of trans-paraconic acids $^{6}$}

A round-bottomed flask equipped with a magnetic stirring bar was charged with paraconic acids 12 (0.6 mmol, 1 equiv) and methoxy magnesium methylcarbonate (Stiles reagent, 11.4 $\mathrm{mL}, 2 \mathrm{M}$ solution in DMF, $22.8 \mathrm{mmol}, 38.0$ equiv) under an inert atmosphere. After stirring at $135{ }^{\circ} \mathrm{C}$ for $60 \mathrm{~h}$, the reaction mixture was acidified with dropwise addition of cold $10 \%$ $\mathrm{HCl}$ at $0{ }^{\circ} \mathrm{C}$. Then DCM $(20 \mathrm{~mL})$ was added to the mixture and stirred for $30 \mathrm{~min}$. The aqueous layer was extracted with DCM $(3 \times 25 \mathrm{~mL})$. The combined organic layers were washed with brine, dried (sodium sulfate) and concentrated in vacuo. The crude compound was used directly for the next reaction. The residue was treated with $9 \mathrm{~mL}$ of a freshly prepared stock solution [HOAc $(24 \mathrm{~mL}), 37 \%$ formaldehyde in water $(18 \mathrm{~mL}), N$ methylaniline $(6 \mathrm{~mL})$ and $\mathrm{NaOAc}(0.72 \mathrm{~g})]$ and stirred for $3 \mathrm{~h}$ at room temperature. Brine solution $(40 \mathrm{~mL}$, containing $1 \mathrm{~mL}$ conc. $\mathrm{HCl})$ was added and the aqueous layer was extracted with $\mathrm{Et}_{2} \mathrm{O}(5 \times 40 \mathrm{~mL})$. The organic fraction was dried (sodium sulfate) and concentrated in vacuo to get crude product which was further purified by flash column chromatography.

\section{(-)-Methylenolactocin (1)}

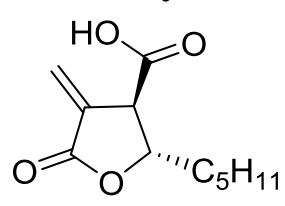


Following the experimental procedure, paraconic acid 12a (120 mg, $0.6 \mathrm{mmol}, 1$ equiv) was converted to product 1 which was purified as a white solid $(73 \mathrm{mg}, 57 \%$ ) from the crude reaction mixture using flash column chromatography [Silica gel, $\mathrm{Et}_{2} \mathrm{O} / \mathrm{hexane} / \mathrm{AcOH}$ (30:69:1)]. $\mathbf{R}_{f}=0.6\left[\mathrm{Et}_{2} \mathrm{O} / \mathrm{hexane} / \mathrm{AcOH}(7: 2.8: 0.2)\right] \cdot[\boldsymbol{\alpha}]_{\mathbf{D}}{ }^{21}=-7.59(c=0.5, \mathrm{MeOH}$ for $96 \%$ $e e)$, lit. $^{2}[\alpha]_{\mathrm{D}}{ }^{26}=-6.7(c=0.5, \mathrm{MeOH})$. M. P.: $83-85{ }^{\circ} \mathrm{C}$, lit. ${ }^{2}$ M. P.: $82-84{ }^{\circ}$ C. ${ }^{1} \mathbf{H}$ NMR $(500$ $\left.\mathrm{MHz}, \mathrm{CDCl}_{3}\right) \delta 6.46(\mathrm{~d}, J=3.0 \mathrm{~Hz}, 1 \mathrm{H}), 6.01(\mathrm{~d}, J=3.0 \mathrm{~Hz}, 1 \mathrm{H}), 4.83-4.79(\mathrm{~m}, 1 \mathrm{H}), 3.63-$ $3.61(\mathrm{~m}, 1 \mathrm{H}), 1.80-1.69(\mathrm{~m}, 2 \mathrm{H}), 1.54-1.38(\mathrm{~m}, 2 \mathrm{H}), 1.36-1.29(\mathrm{~m}, 4 \mathrm{H}), 0.90(\mathrm{t}, J=7.0 \mathrm{~Hz}$, 3H). ${ }^{13} \mathbf{C}$ NMR $\left(125 \mathrm{MHz}, \mathrm{CDCl}_{3}\right) \delta 173.0,168.1,132.6,125.7,78.9,49.5,35.7,31.3,24.4$, 22.4, 13.9. IR (neat) $v$ 3480, 2958, 2931, 2867, 1749, 1660, 1266, 1118, $818 \mathrm{~cm}^{-1}$. HRMS (ESI) $\mathrm{m} / \mathrm{z}$ calcd for $\mathrm{C}_{11} \mathrm{H}_{17} \mathrm{O}_{4}{ }^{+}[\mathrm{M}+\mathrm{H}]^{+} 213.1121$, found 213.1104 .

\section{(+)-Nephrosterinic acid (2)

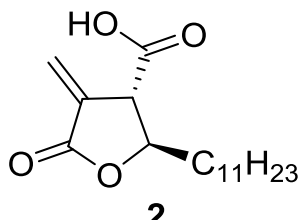

Following the experimental procedure, paraconic acid $\boldsymbol{e n t - 1 2 b}(170 \mathrm{mg}, 0.6 \mathrm{mmol}, 1$ equiv) was converted to product 2 which was purified as a white solid (99 mg, 56\%) from the crude reaction mixture using flash column chromatography [Silica gel, $\mathrm{Et}_{2} \mathrm{O} / \mathrm{h}$ exane/AcOH (30:69:1)]. $\mathbf{R}_{f}=0.6\left[\mathrm{Et}_{2} \mathrm{O} / \mathrm{hexane} / \mathrm{AcOH}(7: 2.8: 0.2)\right] \cdot[\boldsymbol{\alpha}]_{\mathbf{D}}{ }^{23}=+14.0\left(c=0.5, \mathrm{CHCl}_{3}\right.$ for $96 \%$ $e e)$, lit. ${ }^{4}[\alpha]_{\mathrm{D}}{ }^{25}=+12.6\left(c=0.5, \mathrm{CHCl}_{3}\right.$ for $\left.92 \% e e\right)$. M. P.: $79-81{ }^{\circ} \mathrm{C}$, lit. ${ }^{4} \mathrm{M}$. P.: $82-84{ }^{\circ} \mathrm{C}$. ${ }^{1} \mathbf{H}$ NMR $\left(500 \mathrm{MHz}, \mathrm{CDCl}_{3}\right) \delta 6.46(\mathrm{~d}, J=3.0 \mathrm{~Hz}, 1 \mathrm{H}), 6.01(\mathrm{~d}, J=2.5 \mathrm{~Hz}, 1 \mathrm{H}), 4.82-4.79$ (m, 1H), 3.64-3.61 (m, 1H), 1.80-1.68 (m, 2H), 1.54-1.38 (m, 2H), 1.37-1.26 (m, 16H), 0.88 $(\mathrm{t}, J=7.0 \mathrm{~Hz}, 3 \mathrm{H}) .{ }^{13} \mathbf{C}$ NMR $\left(125 \mathrm{MHz}, \mathrm{CDCl}_{3}\right) \delta 173.5,168.1,132.5,125.8,78.8,49.4$, $35.8,31.9,29.6,29.5,29.4,29.3,29.2,24.8,22.7,14.1$. IR (neat) $v$ 3083, 2921, 2849, 1744, 1716, 1661, 1463, 1401, 1256, 963, $712 \mathrm{~cm}^{-1}$. HRMS (ESI) m/z calcd for $\mathrm{C}_{17} \mathrm{H}_{29} \mathrm{O}_{4}{ }^{+}[\mathrm{M}+$ $\mathrm{H}]^{+}$297.2060, found 297.2067.

\section{(+)-Protolichesterinic acid (3)}

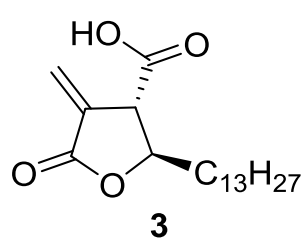

Following the experimental procedure, paraconic acid ent-12c (187 mg, $0.6 \mathrm{mmol}, 1$ equiv) was converted to product 3 which was purified as a white solid (103 $\mathrm{mg}, 53 \%)$ from the crude reaction mixture using flash column chromatography [Silica gel, $\mathrm{Et}_{2} \mathrm{O} / \mathrm{hexane} / \mathrm{AcOH}$ $(20: 80: 1)] . \mathbf{R}_{f}=0.6\left[\mathrm{Et}_{2} \mathrm{O} / \mathrm{hexane} / \mathrm{AcOH}(7: 3: 0.1)\right] \cdot[\boldsymbol{\alpha}]_{\mathbf{D}}{ }^{\mathbf{2 6}}=+12.8\left(c=0.4, \mathrm{CHCl}_{3}\right.$ for $96 \%$ $e e)$, lit. ${ }^{4}[\alpha]_{\mathrm{D}}{ }^{25}=+13.1\left(c=0.4, \mathrm{CHCl}_{3}\right.$ for $\left.93 \% e e\right)$. M. P.: $102-104{ }^{\circ} \mathrm{C}$, lit. ${ }^{4}$ M. P.: $102-104$ ${ }^{\circ} \mathrm{C} .{ }^{1} \mathbf{H}$ NMR $\left(500 \mathrm{MHz}, \mathrm{CDCl}_{3}\right) \delta 6.47(\mathrm{~d}, J=2.5,1 \mathrm{H}), 6.01(\mathrm{~d}, J=2.5,1 \mathrm{H}), 4.82-4.79(\mathrm{~m}$, $1 \mathrm{H}), 3.64-3.62(\mathrm{~m}, 1 \mathrm{H}), 1.80-1.68(\mathrm{~m}, 2 \mathrm{H}), 1.54-1.45(\mathrm{~m}, 1 \mathrm{H}), 1.45-1.38(\mathrm{~m}, 1 \mathrm{H}), 1.37-$ $1.22(\mathrm{~m}, 20 \mathrm{H}), 0.88(\mathrm{t}, J=7.5 \mathrm{~Hz}, 3 \mathrm{H}) .{ }^{13} \mathbf{C}$ NMR $\left(125 \mathrm{MHz}, \mathrm{CDCl}_{3}\right) \delta 173.8,168.1,132.5$, $125.8,78.8,49.5,35.8,31.9,29.7,29.6,29.6,29.5,29.4,29.3,29.2,24.8,22.7,14.1$. IR 
(neat) $v$ 3070, 2955, 2919, 2850, 1743, 1716, 1661, 1466, 1353, 953, 813, $714 \mathrm{~cm}^{-1}$. HRMS (ESI) $\mathrm{m} / \mathrm{z}$ calcd for $\mathrm{C}_{19} \mathrm{H}_{33} \mathrm{O}_{4}{ }^{+}[\mathrm{M}+\mathrm{H}]^{+} 325.2373$, found 325.2374 .

\section{Experimental procedure for $\alpha$-methylation of paraconic acids $\mathbf{3}^{\mathbf{3}}$}

To a solution of paraconic acid 12 or 13 (1.0 mmol, 1.0 equiv) in THF $(15 \mathrm{~mL})$ at $-78{ }^{\circ} \mathrm{C}$ was added NaHMDS (2.2 mL, $2.2 \mathrm{mmol}, 1.0 \mathrm{M}$ solution in THF, 2.2 equiv), and stirred for $1.5 \mathrm{~h}$. MeI ( $622 \mu \mathrm{L}, 10.0 \mathrm{mmol}, 10.0$ equiv) was added and the reaction mixture stirred at -78 ${ }^{\circ} \mathrm{C}$ for $2 \mathrm{~h}$. Then, allowed to warm to $-20{ }^{\circ} \mathrm{C}$ and stirred at this temperature for $2 \mathrm{~h}$. The reaction mixture was quenched with $1 \mathrm{~N} \mathrm{HCl}(10 \mathrm{~mL})$ and extracted with EtOAc $(3 \times 15$ $\mathrm{mL}$ ). The organic fraction was dried (sodium sulfate) and concentrated in vacuo to get crude product which was further purified by flash column chromatography.

\section{(-)-Phaseolinic acid (4)}<smiles>[CH][C@@H]1OC(=O)[C@H](C)[C@H]1C(=O)O</smiles>

Following the experimental procedure, paraconic acids 13a (200 mg, $1.0 \mathrm{mmol}, 1.0$ equiv) was converted to product 4 which was purified as a white solid (199 $\mathrm{mg}, 93 \%)$ from the crude reaction mixture using flash column chromatography [Silica gel, $\mathrm{Et}_{2} \mathrm{O} / \mathrm{hexane} / \mathrm{AcOH}$ (35:64:1)]. $\mathbf{R}_{f}=0.6\left[\mathrm{Et}_{2} \mathrm{O} / \mathrm{h}\right.$ exane/AcOH (7:2.8:0.2)]. $[\boldsymbol{\alpha}]_{\mathbf{D}}{ }^{22}=-96.5\left(c=0.2, \mathrm{CHCl}_{3}\right.$ for $94 \%$ $e e)$, lit. ${ }^{7}[\alpha]_{\mathrm{D}}{ }^{20}=-114.4\left(c=1.46, \mathrm{CHCl}_{3}\right)$. M. P.: $138-140{ }^{\circ} \mathrm{C}$, lit. ${ }^{7}$ M. P.: $136-137{ }^{\circ} \mathrm{C} .{ }^{1} \mathbf{H}$ NMR $\left(500 \mathrm{MHz}, \mathrm{CDCl}_{3}\right) \delta 4.72-4.68(\mathrm{~m}, 1 \mathrm{H}), 3.22(\mathrm{t}, J=9.0 \mathrm{~Hz}, 1 \mathrm{H}) 3.07-3.01(\mathrm{~m}, 1 \mathrm{H})$, $1.63-1.53(\mathrm{~m}, 3 \mathrm{H}), 1.44-1.35(\mathrm{~m}, 1 \mathrm{H}), 1.33-1.27(\mathrm{~m}, 7 \mathrm{H}), 0.88(\mathrm{t}, J=7.0 \mathrm{~Hz}, 3 \mathrm{H}) .{ }^{13} \mathbf{C}$ NMR $\left(125 \mathrm{MHz}, \mathrm{CDCl}_{3}\right) \delta 177.4,174.8,77.4,51.6,36.5,31.3,31.1,25.3,22.4,14.4,13.9$. IR (neat) $v$ 3181, 2956, 1748, 1738, 1249, 1180, 982, $693 \mathrm{~cm}^{-1}$. HRMS (ESI) m/z calcd for $\mathrm{C}_{11} \mathrm{H}_{19} \mathrm{O}_{4}^{+}[\mathrm{M}+\mathrm{H}]^{+}$215.1278, found 215.1257.

\section{(-)-Nephromopsinic acid (5)}<smiles>C[C@@H]1OC(=O)[C@H](C)[C@@H]1C(=O)O</smiles>

Following the experimental procedure, paraconic acid 13c (312 mg, $1.0 \mathrm{mmol}, 1.0$ equiv) was converted to product 5 which was purified as a white solid $(312 \mathrm{mg}, 96 \%)$ from the crude reaction mixture using flash column chromatography [Silica gel, $\mathrm{Et}_{2} \mathrm{O} / \mathrm{hexane} / \mathrm{AcOH}$ (40:60:1)]. $\mathbf{R}_{f}=0.5\left[\mathrm{Et}_{2} \mathrm{O} / \mathrm{hexane} / \mathrm{AcOH}(7: 3: 0.1)\right] \cdot[\boldsymbol{\alpha}]_{\mathbf{D}}{ }^{\mathbf{2 6}}=-85.7\left(c=0.5, \mathrm{CHCl}_{3}\right.$ for $95 \%$ $e e)$, lit. ${ }^{8}[\alpha]_{\mathrm{D}}{ }^{20}=-84\left(c=0.25, \mathrm{CHCl}_{3}\right)$. M. P.: $134-136{ }^{\circ} \mathrm{C}$, lit. ${ }^{8}$ M. P.: $136{ }^{\circ} \mathrm{C} .{ }^{1} \mathbf{H} \mathbf{~ N M R}$ $\left(500 \mathrm{MHz}, \mathrm{CDCl}_{3}\right) \delta 4.72-4.67(\mathrm{~m}, 1 \mathrm{H}), 3.22(\mathrm{t}, J=9.5 \mathrm{~Hz}, 1 \mathrm{H}), 3.07-3.00(\mathrm{~m}, 1 \mathrm{H}), 1.63-$ $1.50(\mathrm{~m}, 3 \mathrm{H}), 1.40-1.25(\mathrm{~m}, 24 \mathrm{H}), 0.88(\mathrm{t}, J=7.0 \mathrm{~Hz}, 3 \mathrm{H}) .{ }^{13} \mathbf{C ~ N M R}\left(125 \mathrm{MHz}, \mathrm{CDCl}_{3}\right) \delta$ 177.4, 174.7, 77.3, 51.6, 36.5, 31.9, 31.2, 29.7, 29.6, 29.6, 29.5, 29.4, 29.3, 29.2, 25.6, 22.7, 
14.4, 14.1. IR (neat) $v$ 2918, 2850, 1746, 1470, 1423, 1202, 1181, 980, $697 \mathrm{~cm}^{-1}$. HRMS (ESI) calcd for $\mathrm{C}_{19} \mathrm{H}_{35} \mathrm{O}_{4}{ }^{+}[\mathrm{M}+\mathrm{H}]^{+}$327.2530, found 327.2510.

\section{(+)-Nephrosteranic acid (6)}<smiles>[CH][C@@H]1OC(=O)[C@H](C)[C@H]1C(=O)O</smiles>

Following the experimental procedure, paraconic acid ent-12b (284 mg, $1.0 \mathrm{mmol}, 1.0$ equiv) was converted to product 6 which was purified as a white solid (265 $\mathrm{mg}, 89 \%$ ) from the crude reaction mixture using flash column chromatography [Silica gel, $\mathrm{Et}_{2} \mathrm{O} / \mathrm{hexane} / \mathrm{AcOH}$ (30:69:1)]. $\mathbf{R}_{f}=0.6\left[\mathrm{Et}_{2} \mathrm{O} /\right.$ hexane/AcOH $\left.(7: 2.8: 0.2)\right] .[\boldsymbol{\alpha}]_{\mathbf{D}}{ }^{23}=+23.3\left(c=0.2, \mathrm{CHCl}_{3}\right.$ for $96 \%$ $e e)$, lit. ${ }^{9}[\alpha]_{\mathrm{D}}{ }^{25}+27.2\left(c=1.45, \mathrm{CHCl}_{3}\right)$. M. P.: $96-98{ }^{\circ} \mathrm{C}$, lit. ${ }^{9}$ M. P.: $96-98{ }^{\circ} \mathrm{C} .{ }^{\mathbf{1}} \mathbf{H}$ NMR $\left(500 \mathrm{MHz}, \mathrm{CDCl}_{3}\right) \delta 4.50-4.46(\mathrm{~m}, 1 \mathrm{H}), 3.01-2.95(\mathrm{~m}, 1 \mathrm{H}), 2.69(\mathrm{dd}, J=11.5,9.5 \mathrm{~Hz}, 1 \mathrm{H})$, $1.86-1.79(\mathrm{~m}, 1 \mathrm{H}), 1.75-1.67(\mathrm{~m}, 1 \mathrm{H}), 1.57-1.48(\mathrm{~m}, 1 \mathrm{H}), 1.44-1.26(\mathrm{~m}, 20 \mathrm{H}), 0.88$ (t, $J=$ $6.5 \mathrm{~Hz}, 3 \mathrm{H}) .{ }^{13} \mathrm{C}$ NMR $\left(125 \mathrm{MHz}, \mathrm{CDCl}_{3}\right) \delta 176.6,174.8,79.4,53.8,39.8,34.9,31.9,29.6$, 29.5, 29.4, 29.3, 29.2, 25.3, 22.7, 14.5, 14.1. IR (neat) $v$ 3277, 2917, 2850, 1745, 1716, 1470, 1258, 1200, 963, $696 \mathrm{~cm}^{-1}$. HRMS (ESI) m/z calcd for $\mathrm{C}_{17} \mathrm{H}_{31} \mathrm{O}_{4}{ }^{+}[\mathrm{M}+\mathrm{H}]^{+} 299.2217$, found 299.2215 .

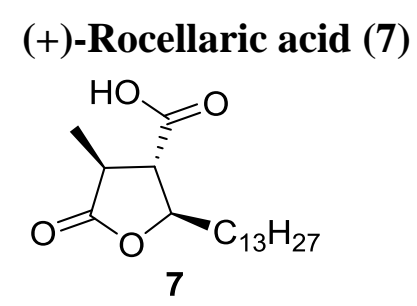

Following the experimental procedure, paraconic acid ent-12c (312 $\mathrm{mg}, 1.0 \mathrm{mmol}, 1.0$ equiv), was converted to product 7 which was purified as a white solid (287 $\mathrm{mg}, 88 \%$ ) from the crude reaction mixture using flash column chromatography [Silica gel,

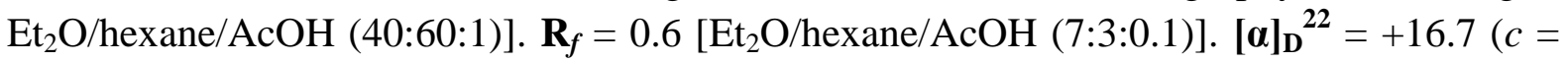
$0.2, \mathrm{CHCl}_{3}$, for $96 \%$ ee $)$, lit. ${ }^{9}[\alpha]_{\mathrm{D}}{ }^{25}+27.0\left(c=0.87, \mathrm{CHCl}_{3}\right)$. M. P.: $102-104{ }^{\circ} \mathrm{C}$, lit. ${ }^{9}$ M. P.: 107-108 ${ }^{\circ} \mathrm{C} .{ }^{1} \mathbf{H}$ NMR $\left(500 \mathrm{MHz}, \mathrm{CDCl}_{3}\right) \delta 4.50-4.46(\mathrm{~m}, 1 \mathrm{H}), 3.01-2.95(\mathrm{~m}, 1 \mathrm{H}), 2.70(\mathrm{dd}$, $J=11.5,9.5 \mathrm{~Hz}, 1 \mathrm{H}), 1.86-1.79(\mathrm{~m}, 1 \mathrm{H}), 1.75-1.67(\mathrm{~m}, 1 \mathrm{H}), 1.57-1.48(\mathrm{~m}, 1 \mathrm{H}), 1.37(\mathrm{~d}, J=$ 7.0, 3H), 1.31-1.22 (m, 21H), $0.88(\mathrm{t}, J=7.0 \mathrm{~Hz}, 3 \mathrm{H}) .{ }^{13} \mathbf{C ~ N M R}\left(125 \mathrm{MHz}, \mathrm{CDCl}_{3}\right) \delta 176.5$, 175.0, 79.3, 53.8, 39.9, 35.0, 31.9, 29.7, 29.7, 29.6, 29.5, 29.4, 29.4, 29.2, 25.3, 22.7, 14.5, 14.1. IR (neat) $v$ 3069, 2915, 2851, 1748, 1713, 1477, 1267, 1208, 1170, 978, $703 \mathrm{~cm}^{-1}$. HRMS (ESI) $\mathrm{m} / \mathrm{z}$ calcd for $\mathrm{C}_{19} \mathrm{H}_{35} \mathrm{O}_{4}{ }^{+}[\mathrm{M}+\mathrm{H}]^{+}$327.2530, found 327.2532.

\section{References}

1) Commercially available triazolium salt cat-II (CAS no. 862095-91-8) has been easily accessed following the literature procedure: Kerr, M. S.; Read de Alaniz, J.; Rovis, T. $J$. Org. Chem., 2005, 70, 5725-5728.

2) De Azevedo, M. B. M.; Murta, M. M.; Greene, A. E. J. Org. Chem. 1992, 57, 4567-4570. 
3) Barros, M. T.; Maycock, C. D.; Ventura, M. R. Org. Lett. 2003, 5, 4097-4099.

4) Nallasivam, J. L.; Fernandes, R. A. Org. Biomol. Chem. 2017, 15, 708-716.

5) Fernandes, R. A.; Chowdhury, A. K. Tetrahedron Asymmetry 2011, 22, 1114-1119.

6) Blanc, D.; Madec, J.; Popowyck, F.; Ayad, T.; Phansavath, P.; Ratovelomanana-Vidal, V.; Genêt, J. P. Adv. Synth. Catal. 2007, 349, 943-950.

7) Amador, M.; Ariza, X.; Garcia, J.; Ortiz, J. J. Org. Chem. 2004, 69, 8172-8175.

8) Mulzer, J.; Kattner, L.; Strecker, A. R.; Schröder, C.; Buschmann, J.; Lehmann, C.; Luger, P. J. Am. Chem. Soc 1991, 72, 4218-4229.

9) Takahata, H.; Uchida, Y.; Momose, T. J. Org. Chem. 1995, 60, 5628-5633. 


\section{NMR and HPLC data}

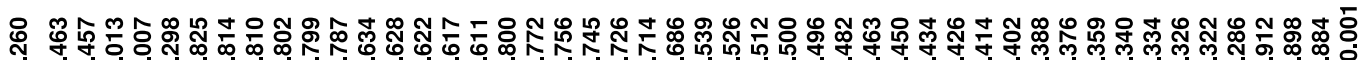

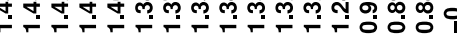

110

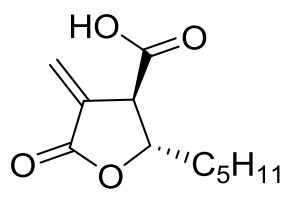

1, $\mathrm{CDCl}_{3}, 500 \mathrm{MHz}$

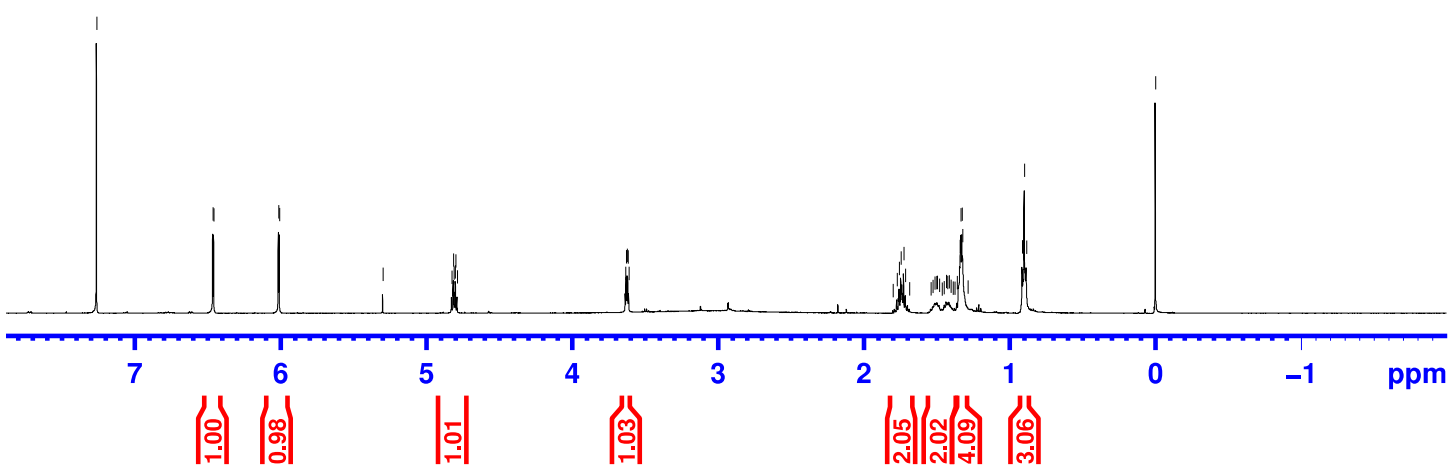

|

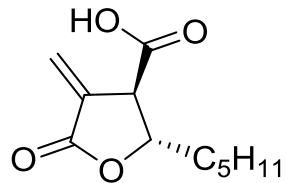

1, $\mathrm{CDCl}_{3}, 125 \mathrm{MHz}$

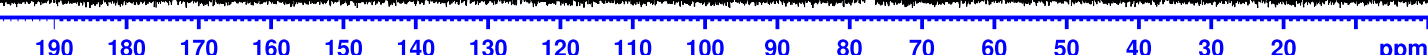



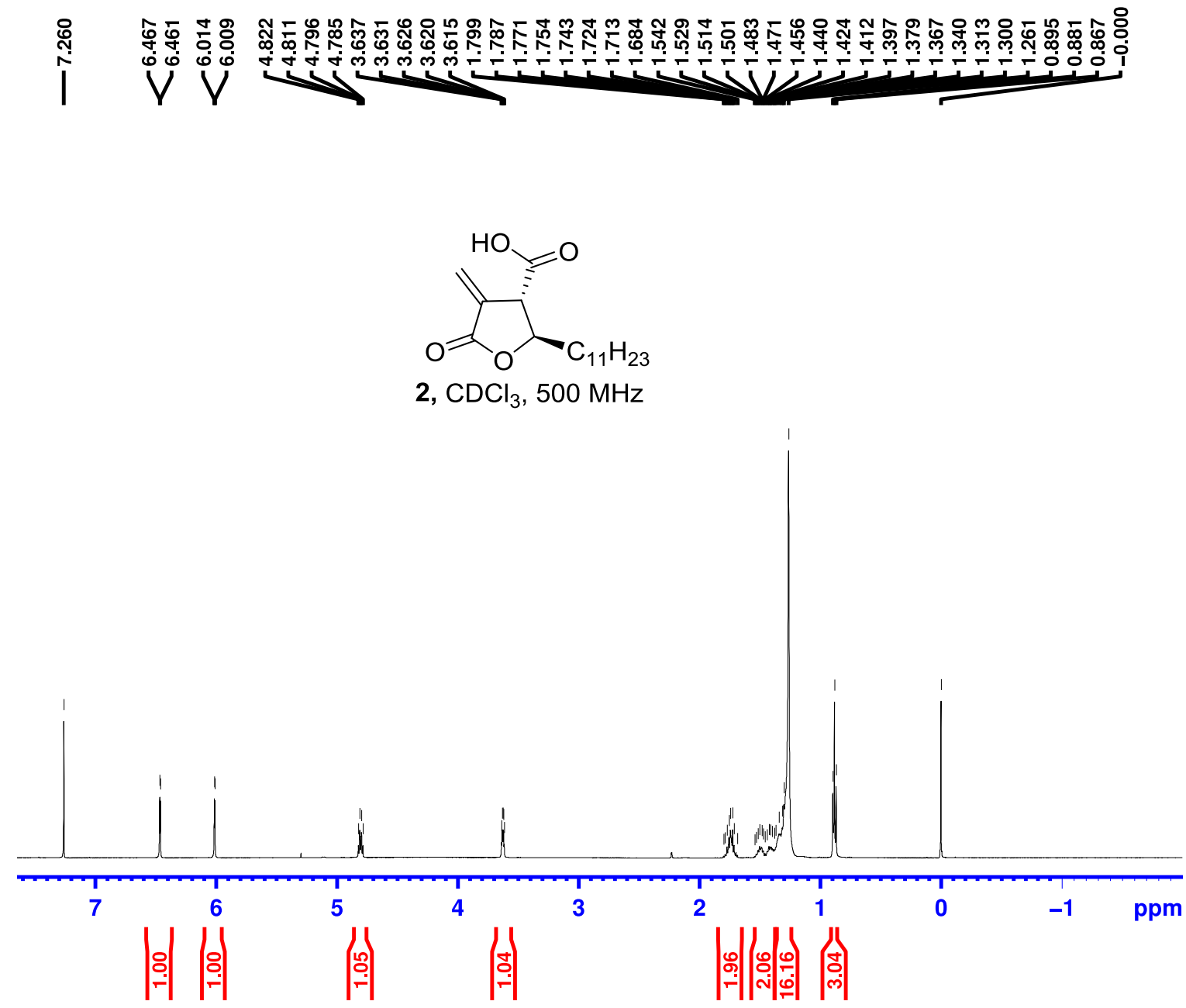

l|

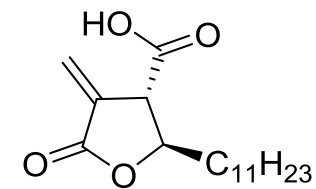

2, $\mathrm{CDCl}_{3}, 125 \mathrm{MHz}$

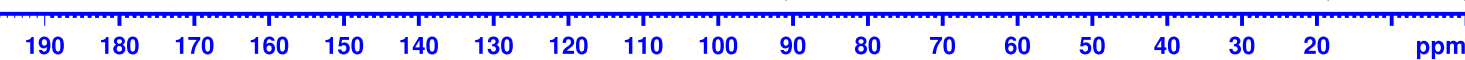



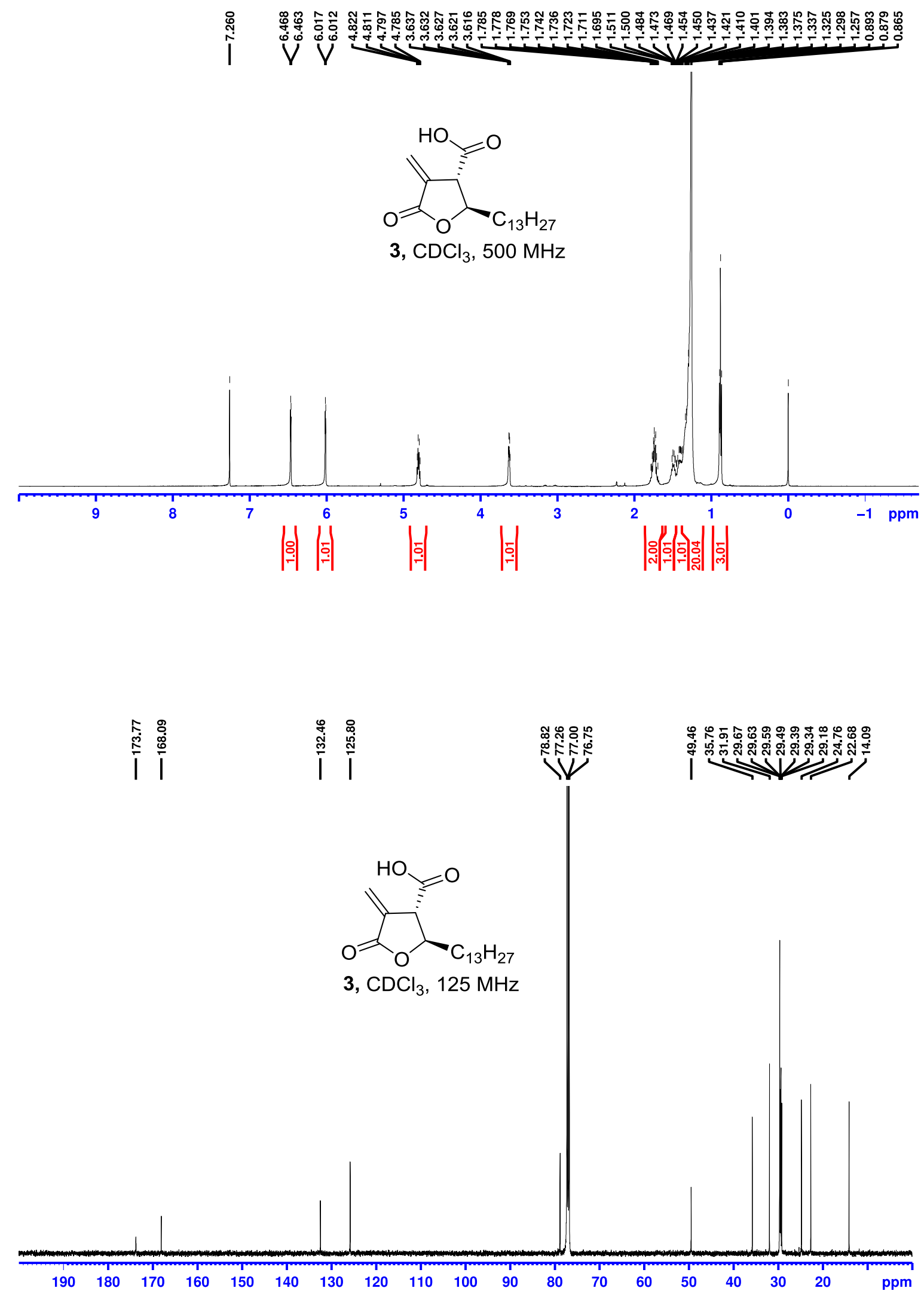

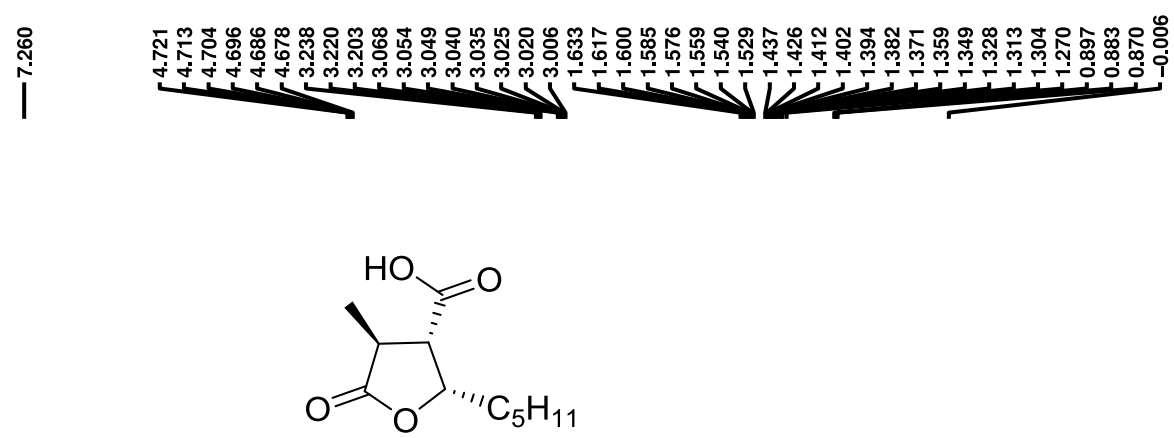

4, $\mathrm{CDCl}_{3}, 500 \mathrm{MHz}$

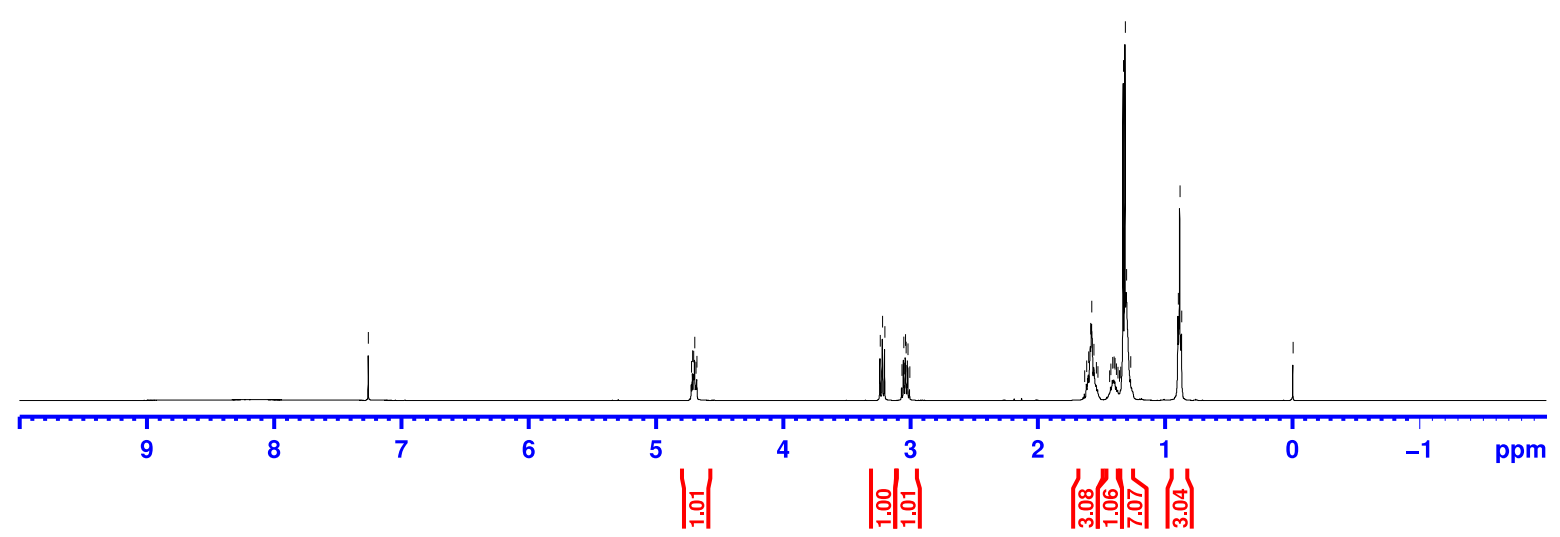

|
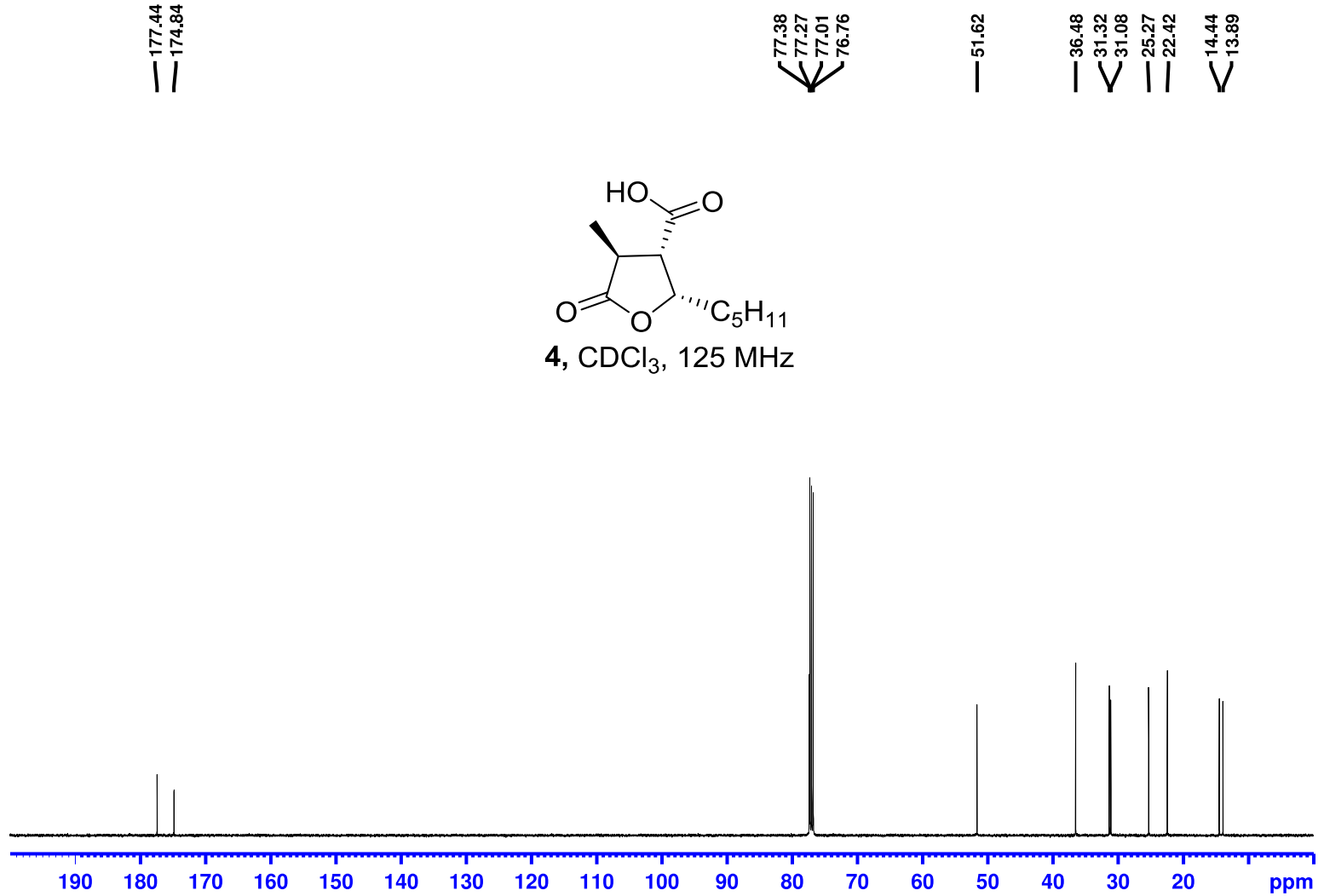

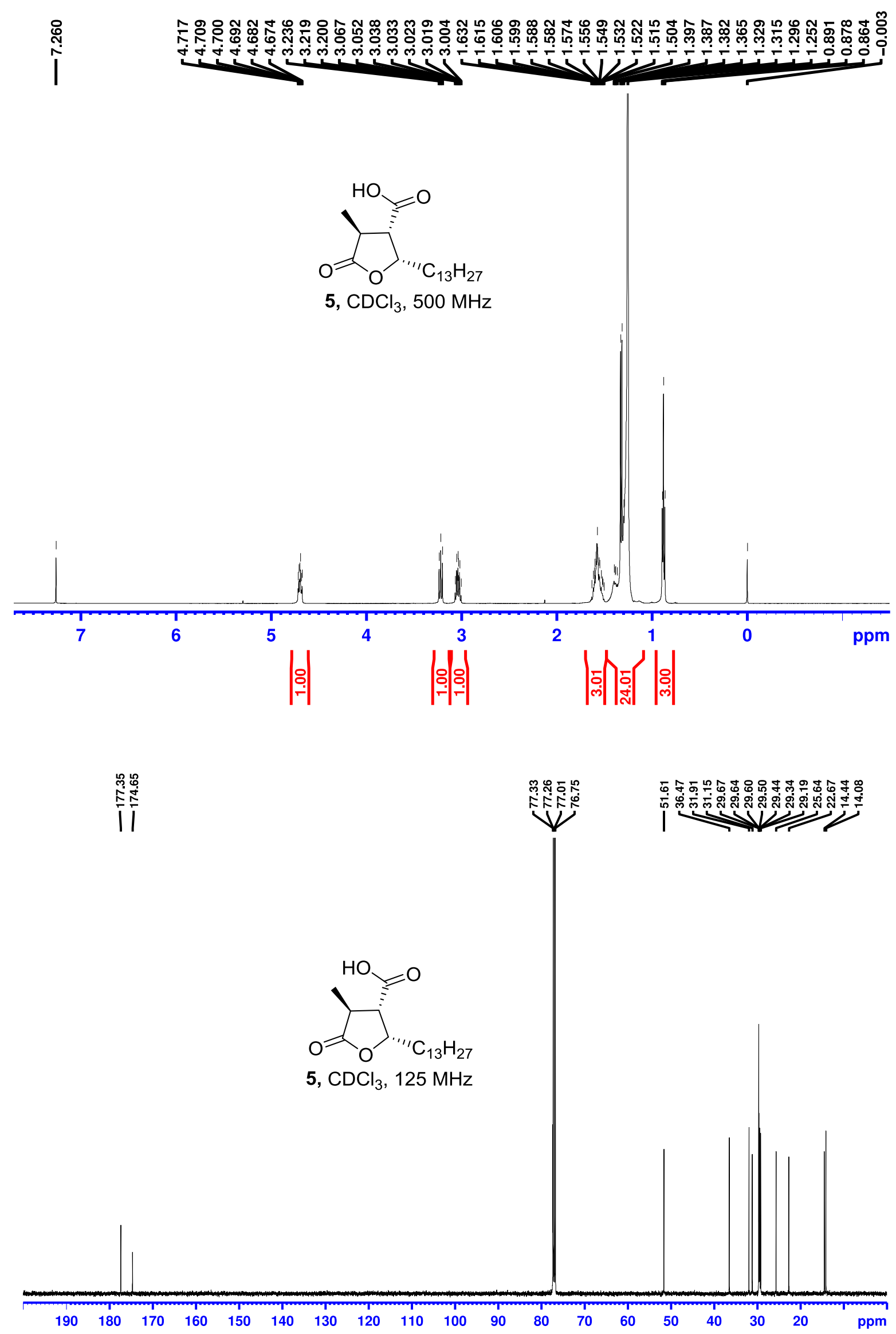


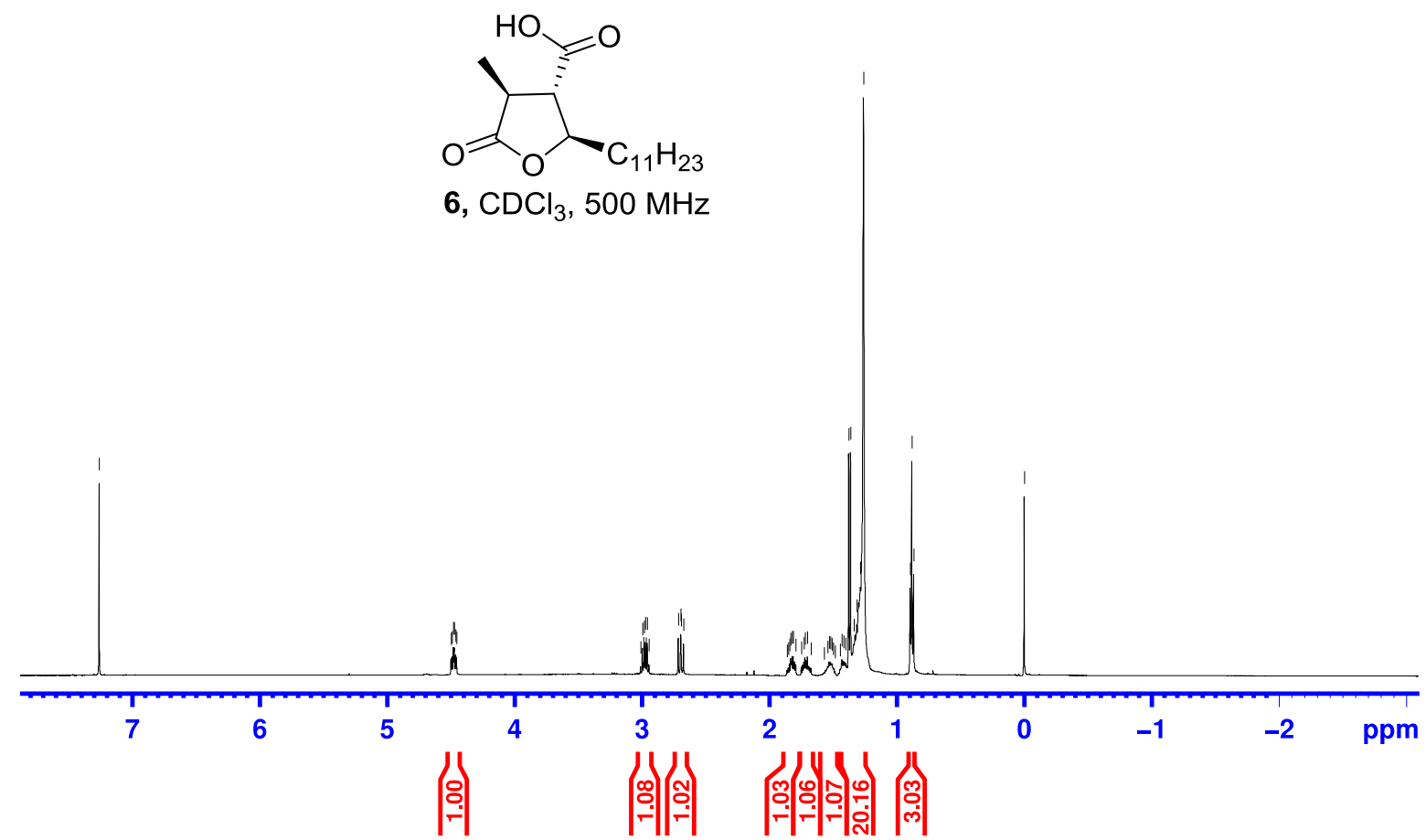

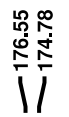
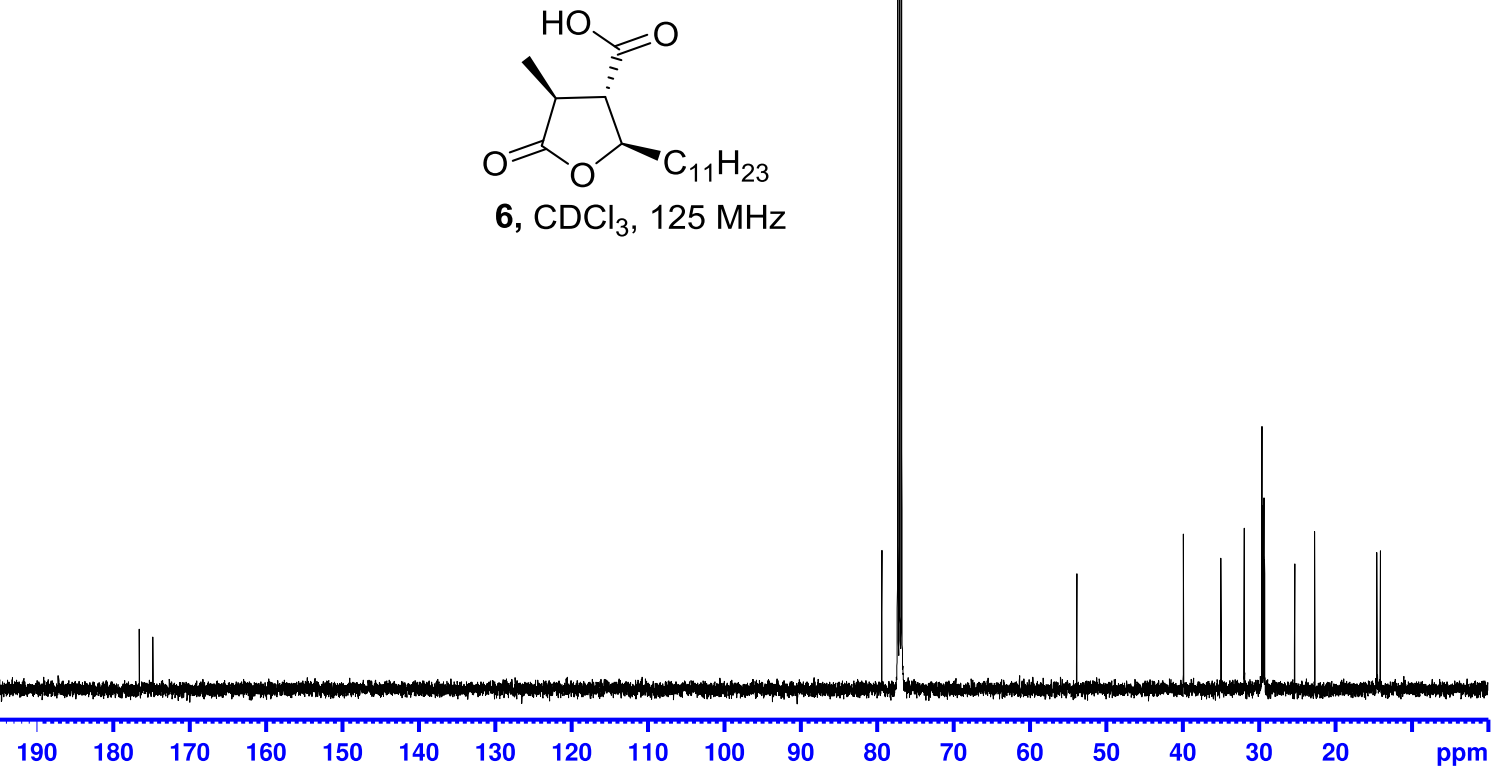


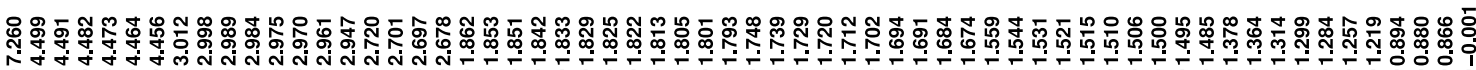
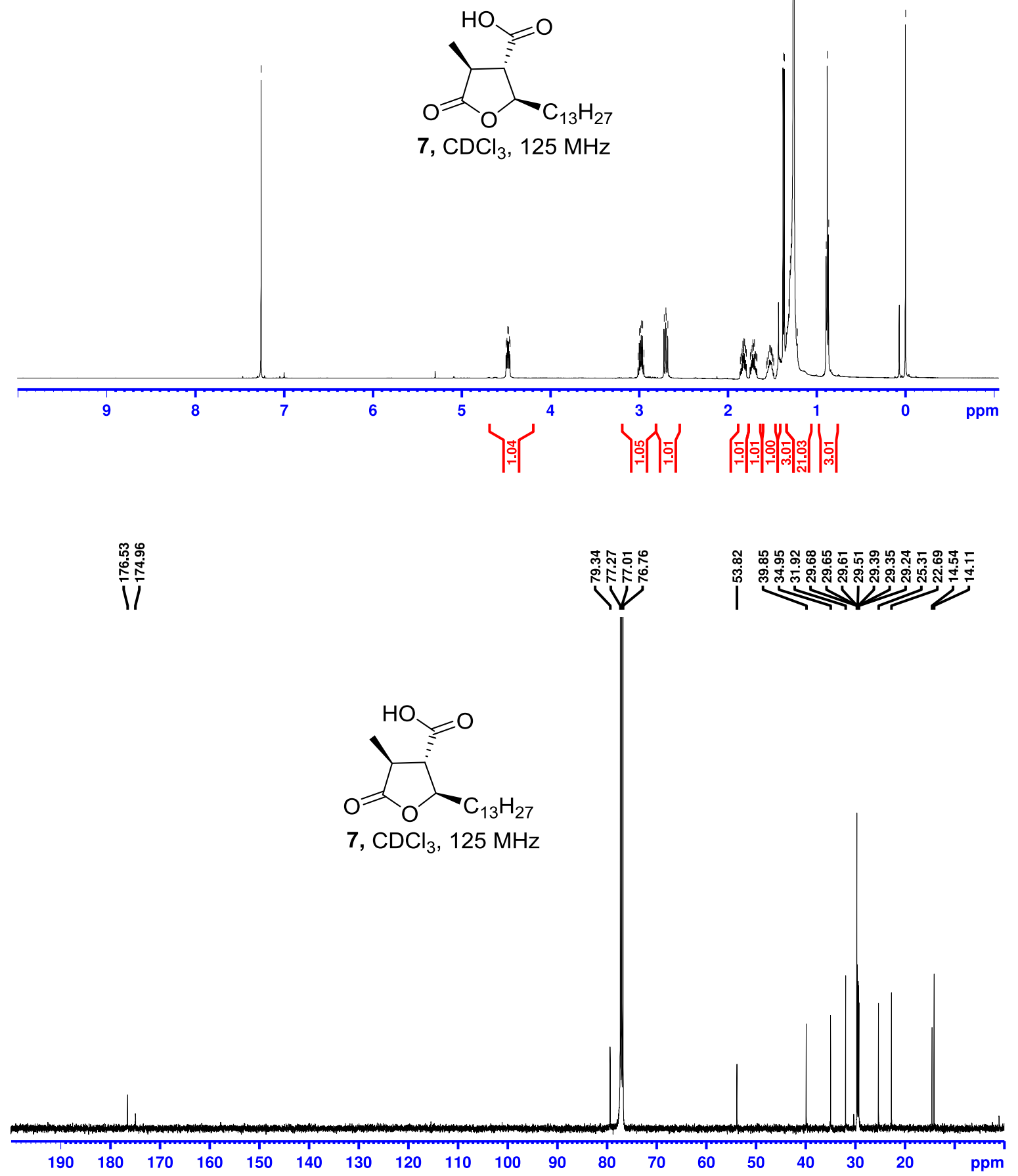


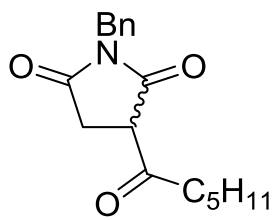

10a, $\mathrm{CDCl}_{3}, 500 \mathrm{MHz}$
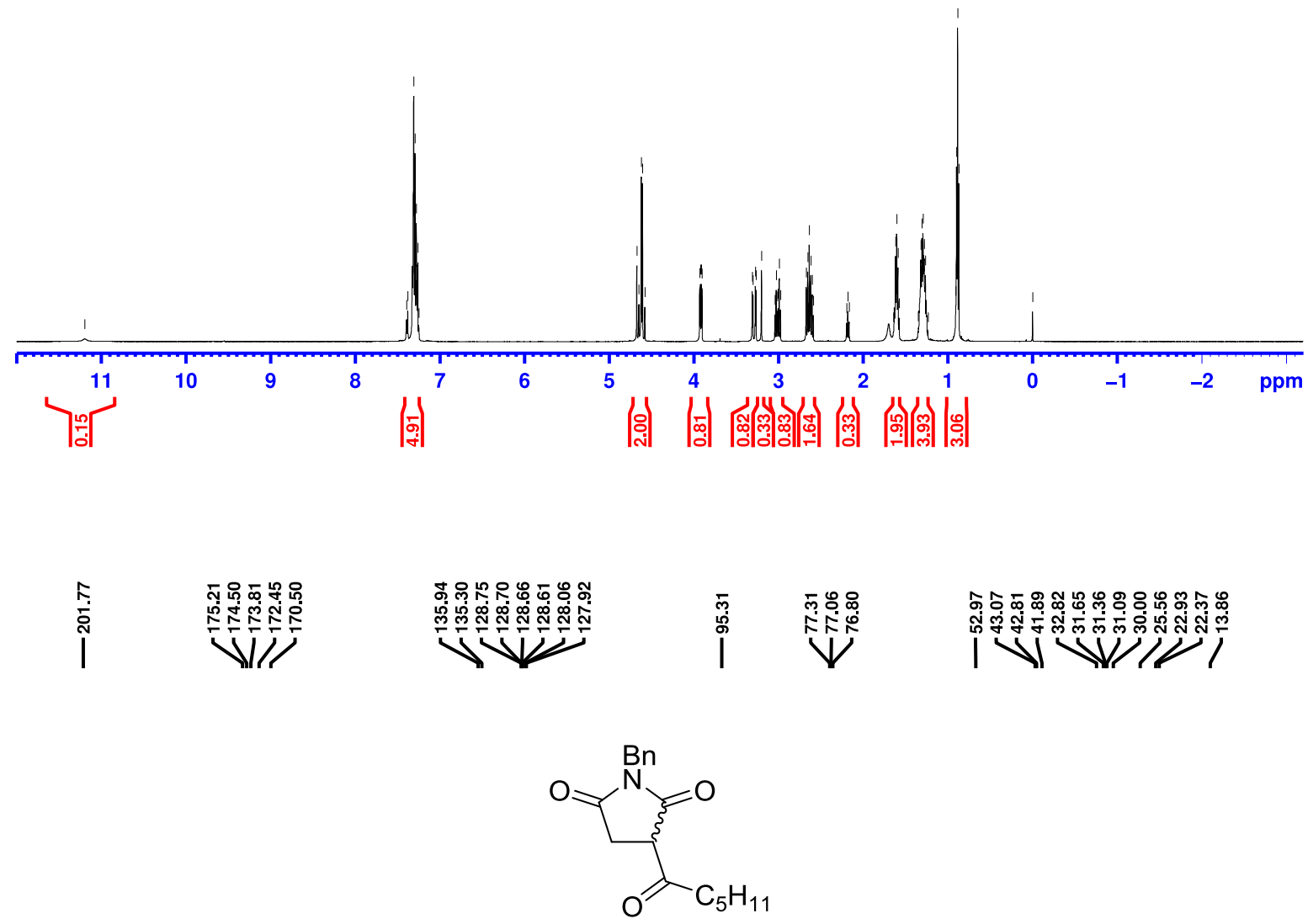

10a, $\mathrm{CDCl}_{3}, 125 \mathrm{MHz}$

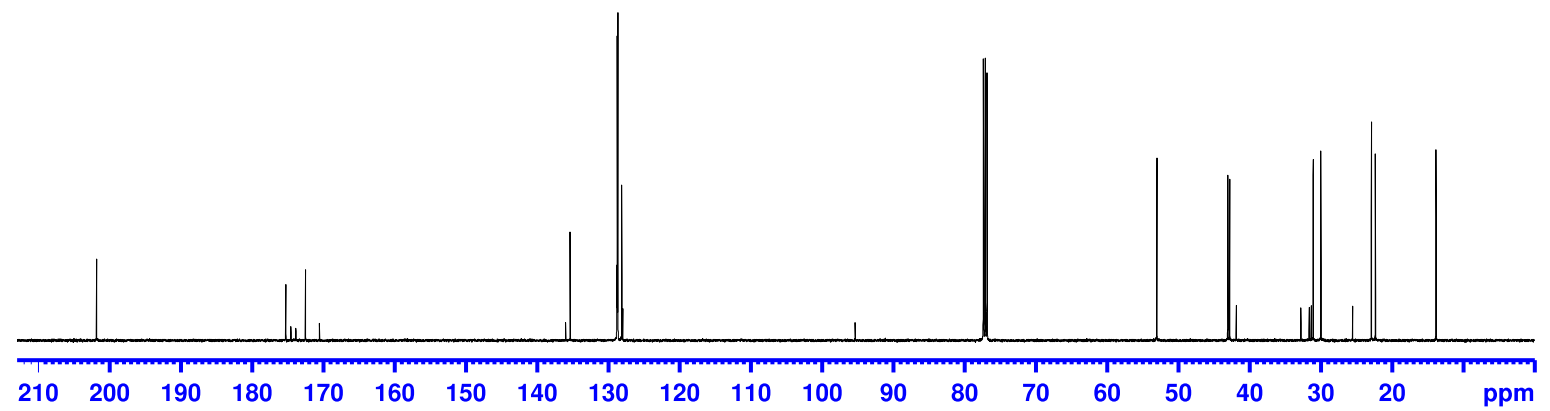




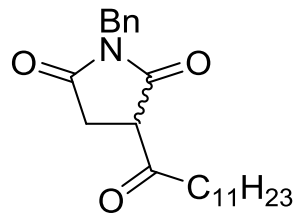

10b, $\mathrm{CDCl}_{3}, 500 \mathrm{MHz}$

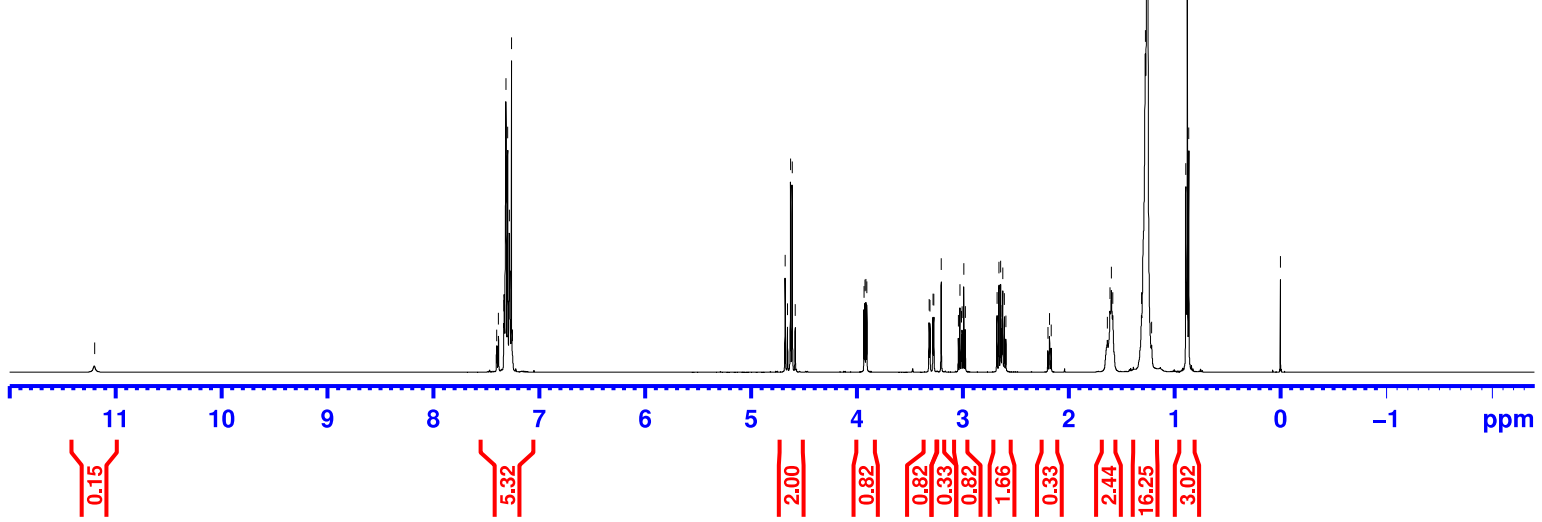

|

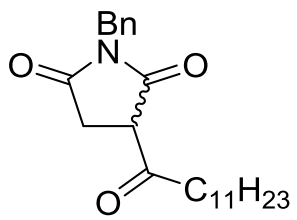

10b, $\mathrm{CDCl}_{3}, 125 \mathrm{MHz}$

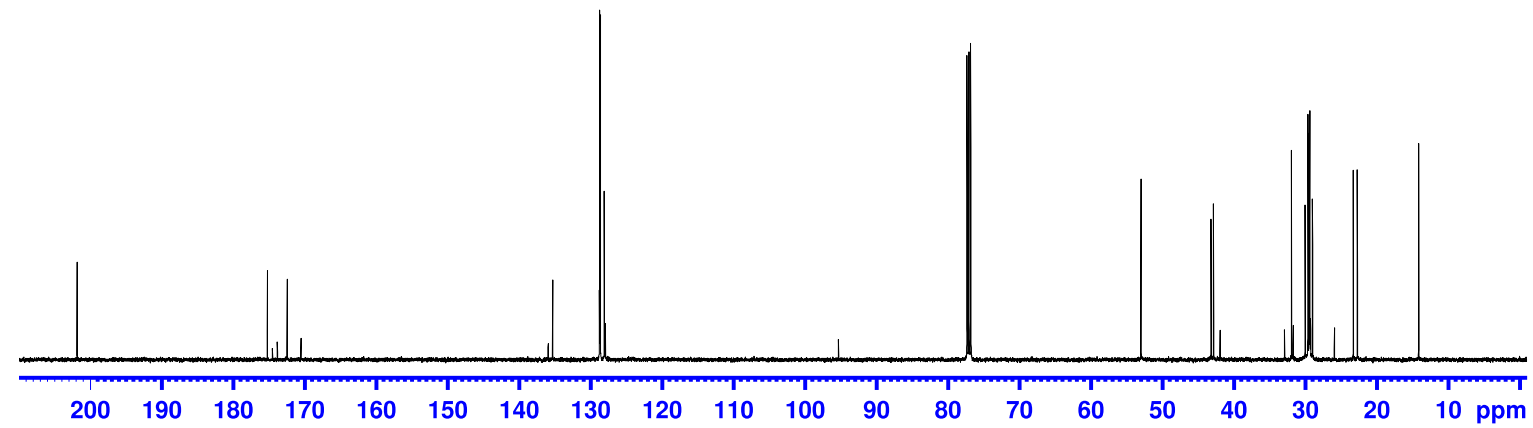



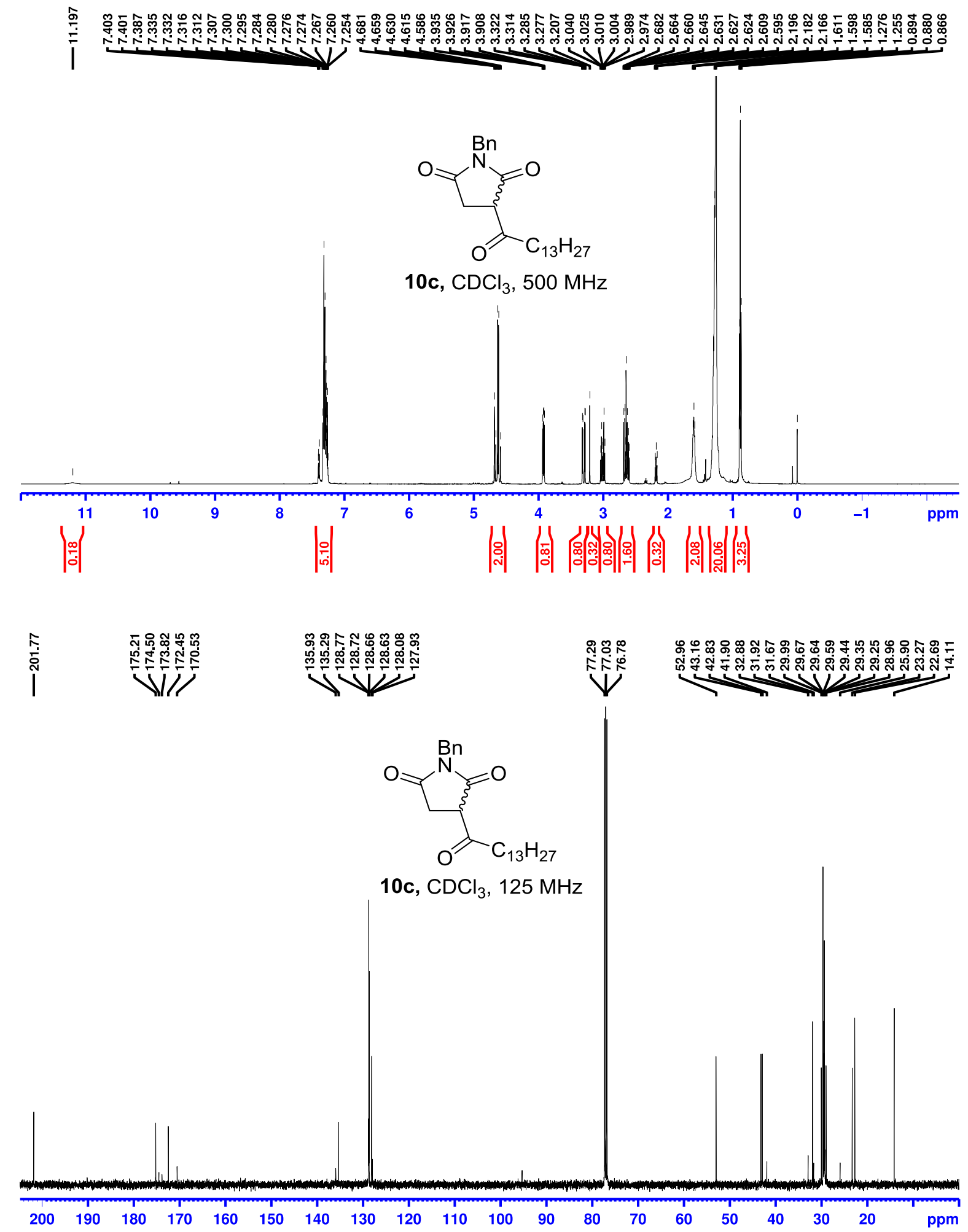


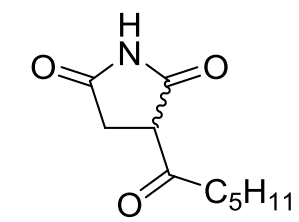

10d, $\mathrm{CDCl}_{3}, 500 \mathrm{MHz}$
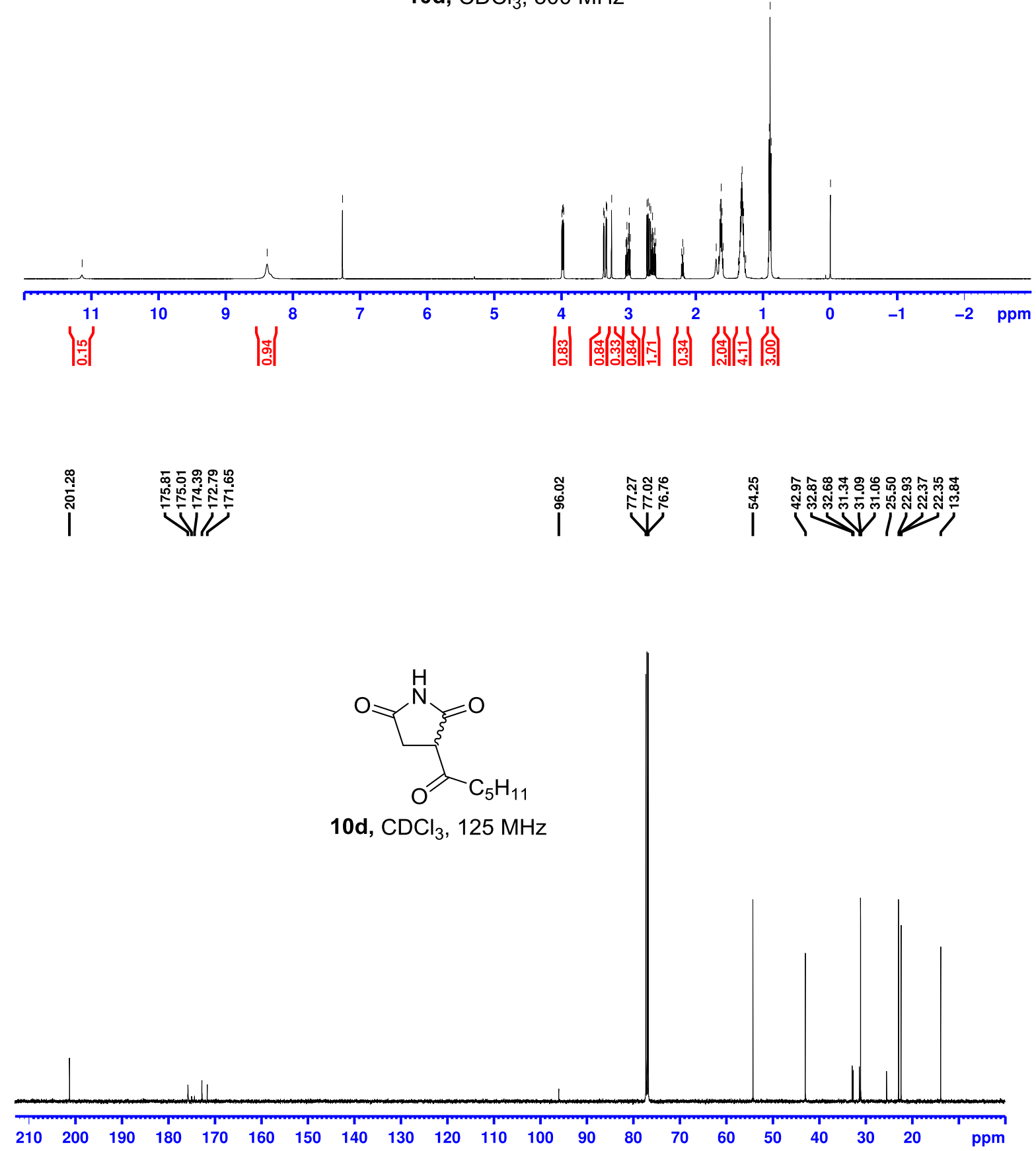


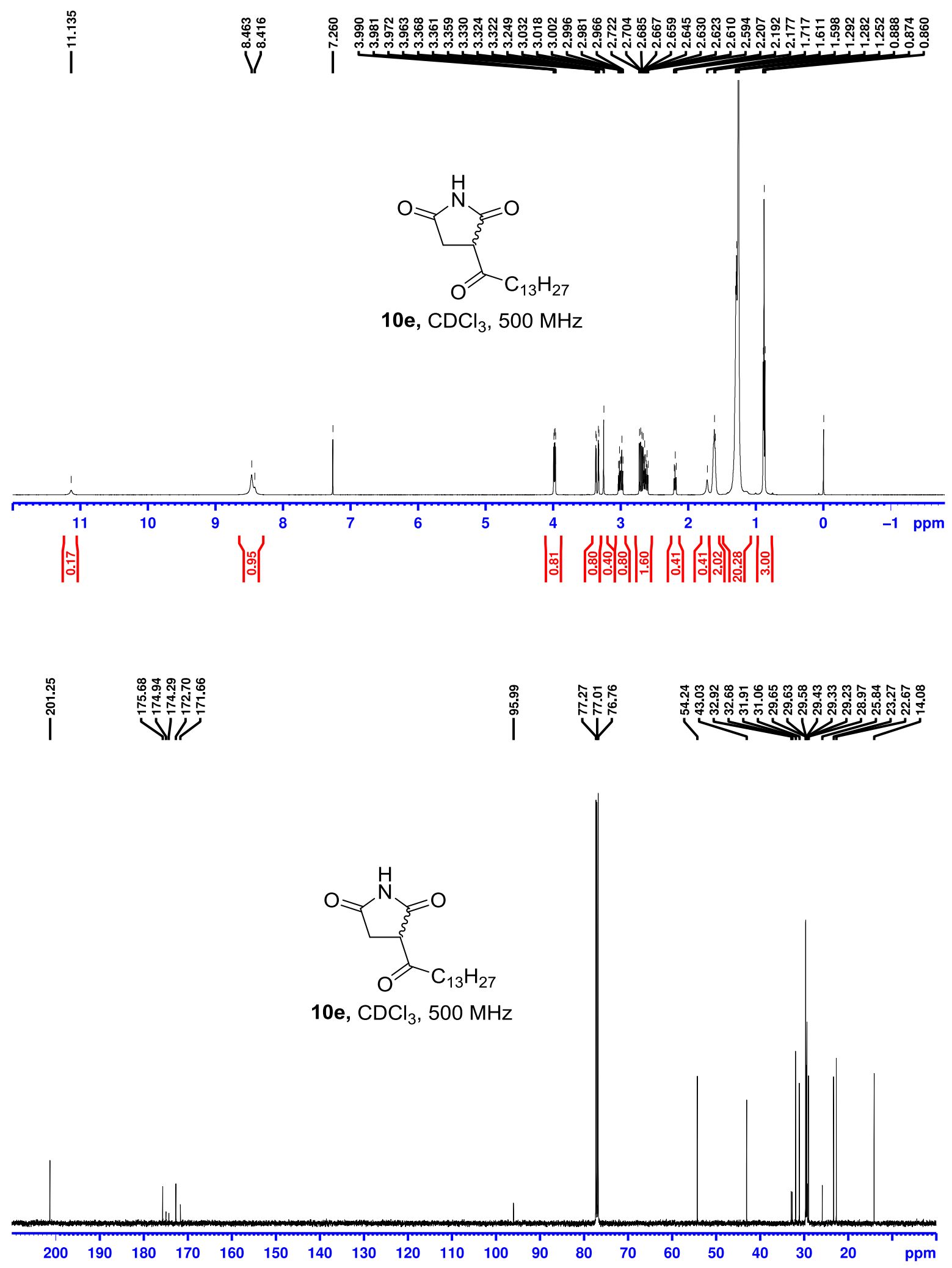




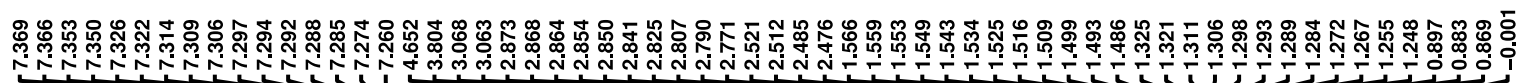

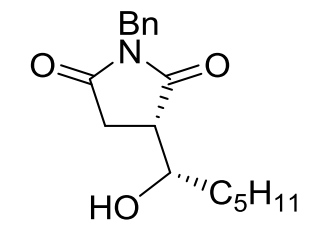

11a, $\mathrm{CDCl}_{3}, 500 \mathrm{MHz}$
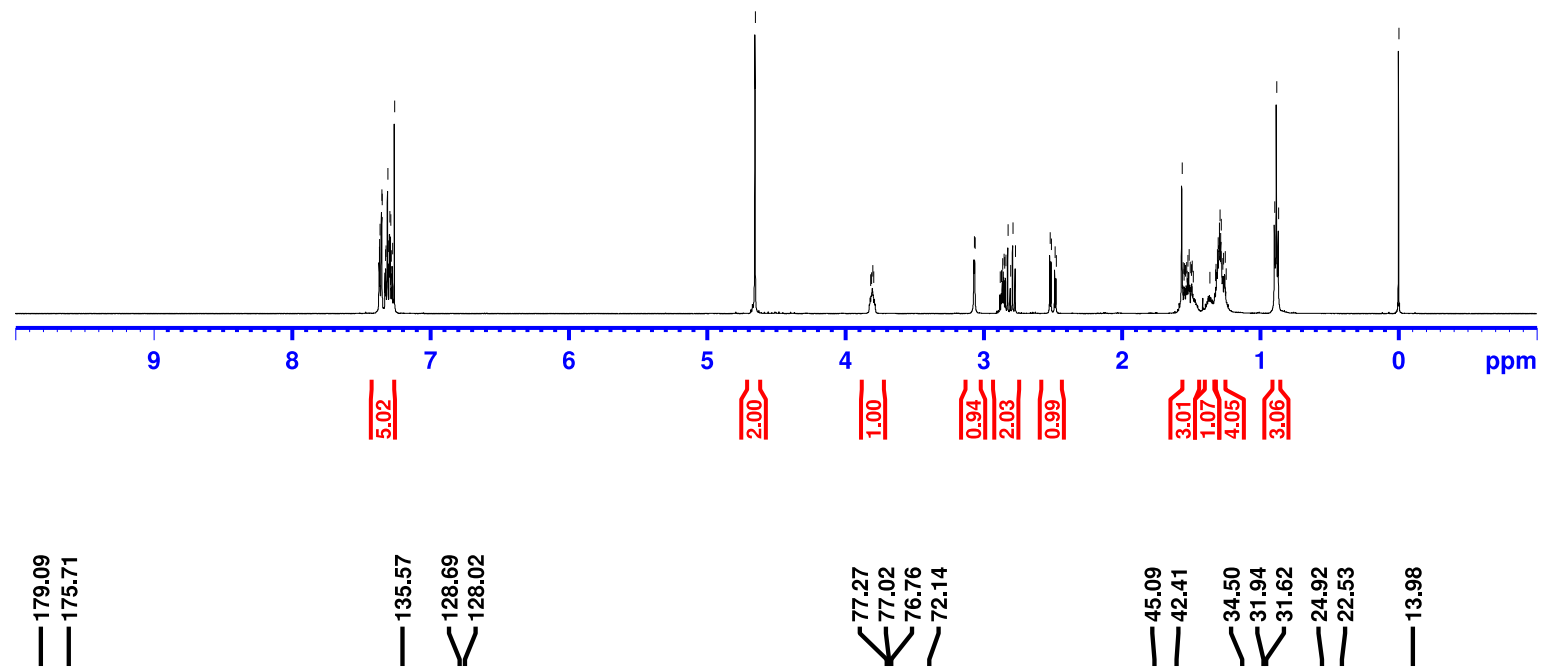

|

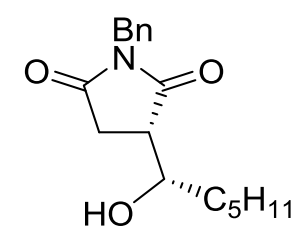

11a, $\mathrm{CDCl}_{3}, 125 \mathrm{MHz}$

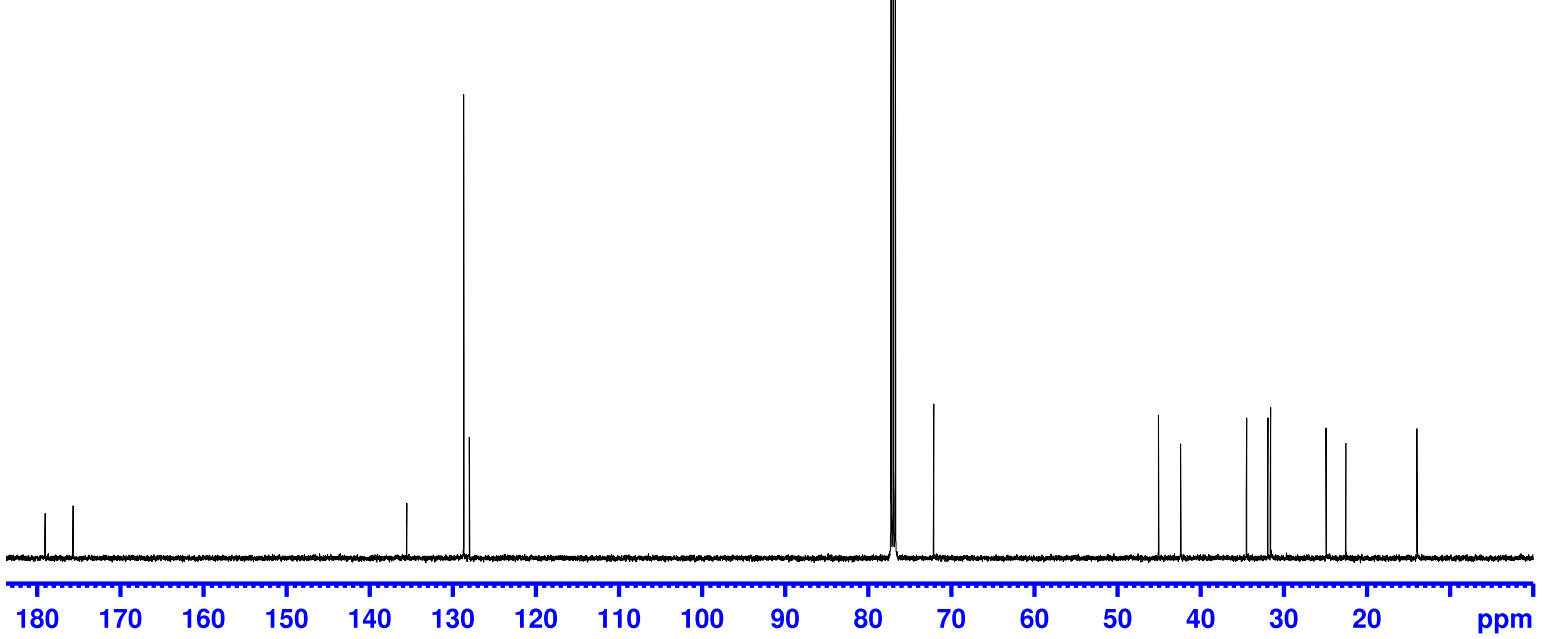



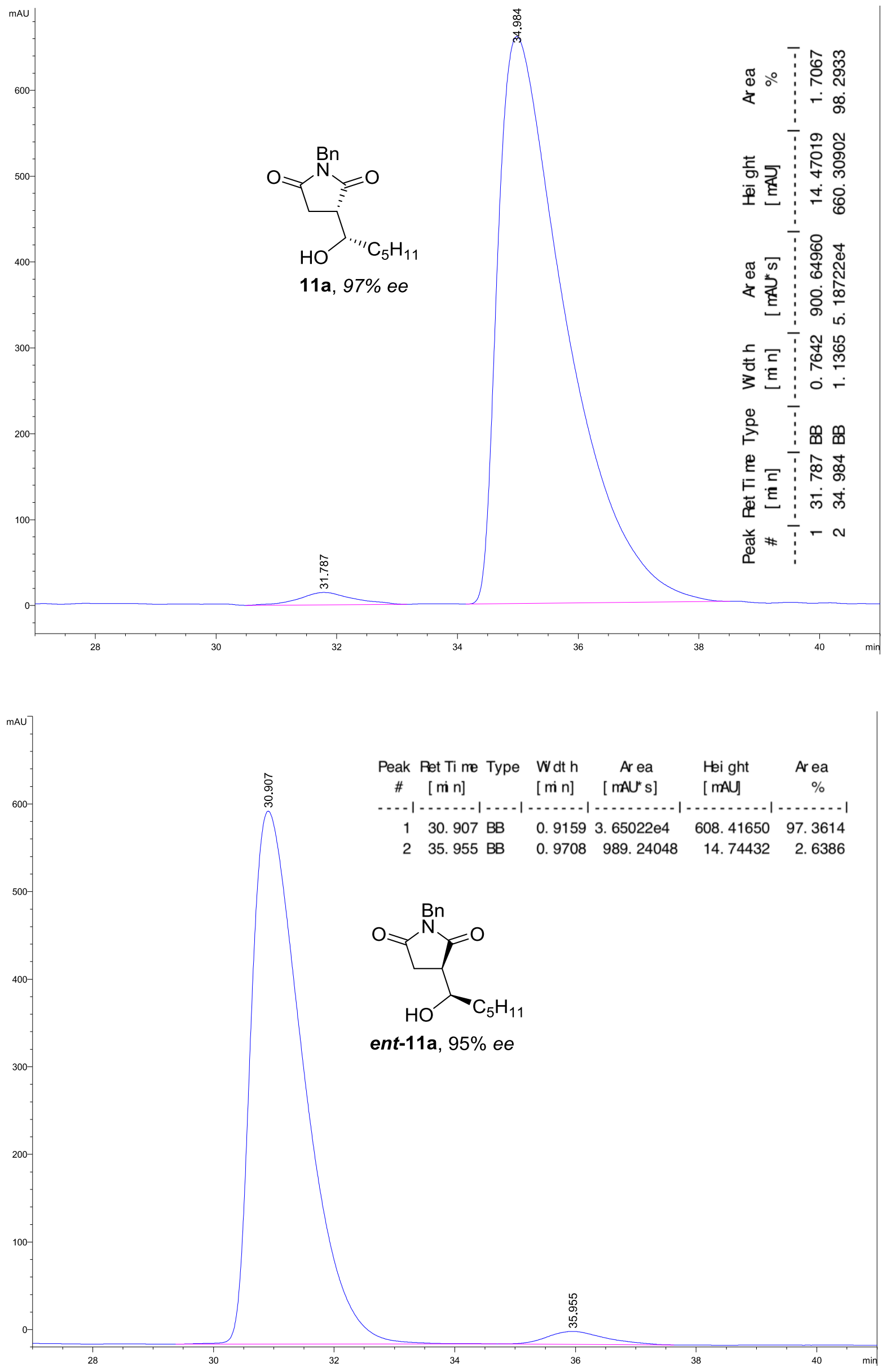


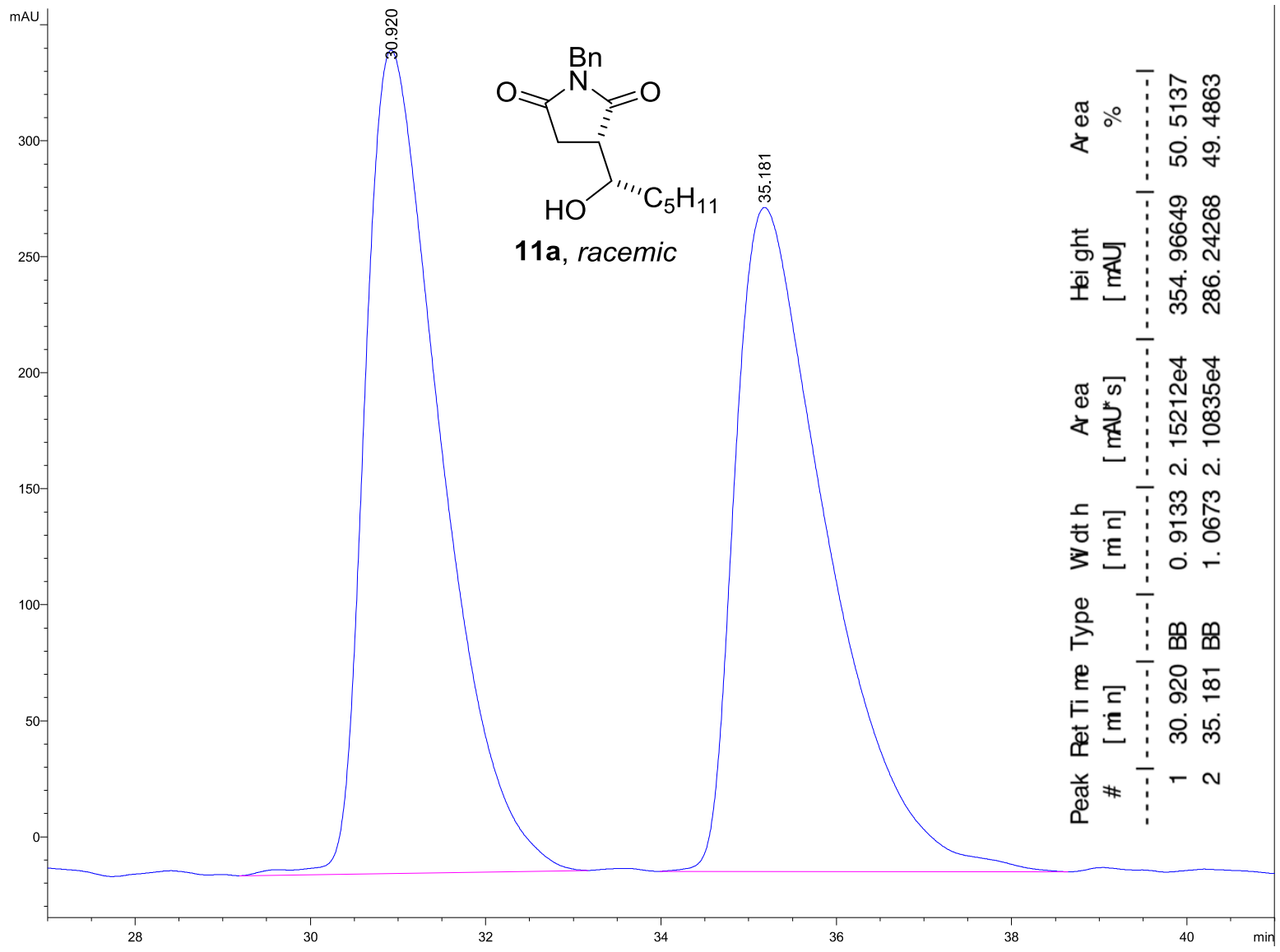

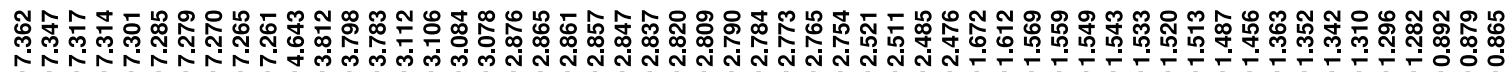
Nin

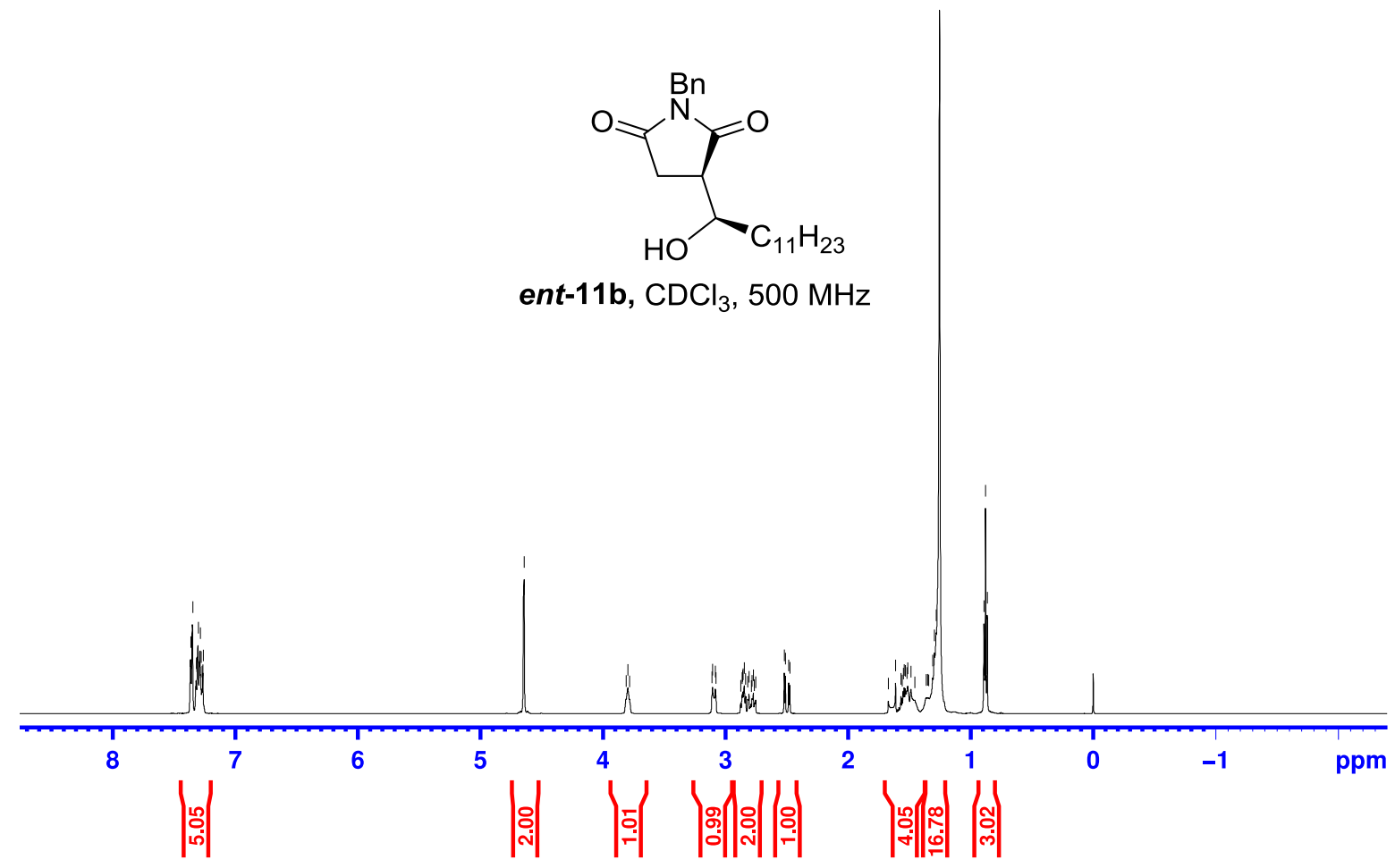




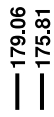

|

ฟ

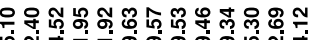

$\underbrace{\underbrace{3}}$

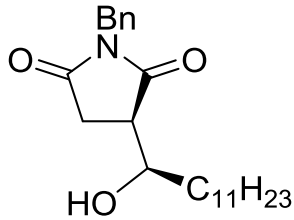

ent-11b, $\mathrm{CDCl}_{3}, 125 \mathrm{MHz}$
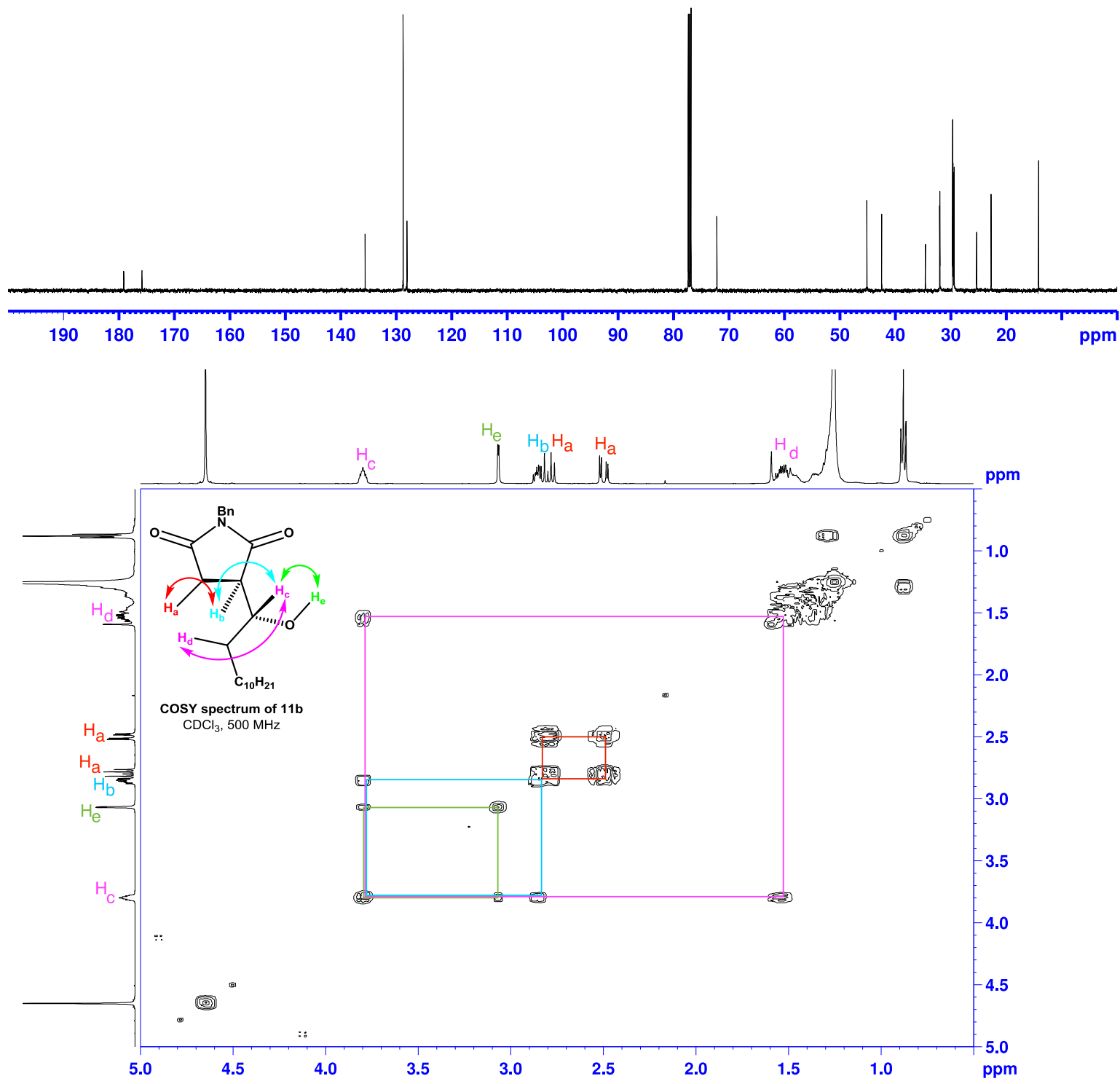

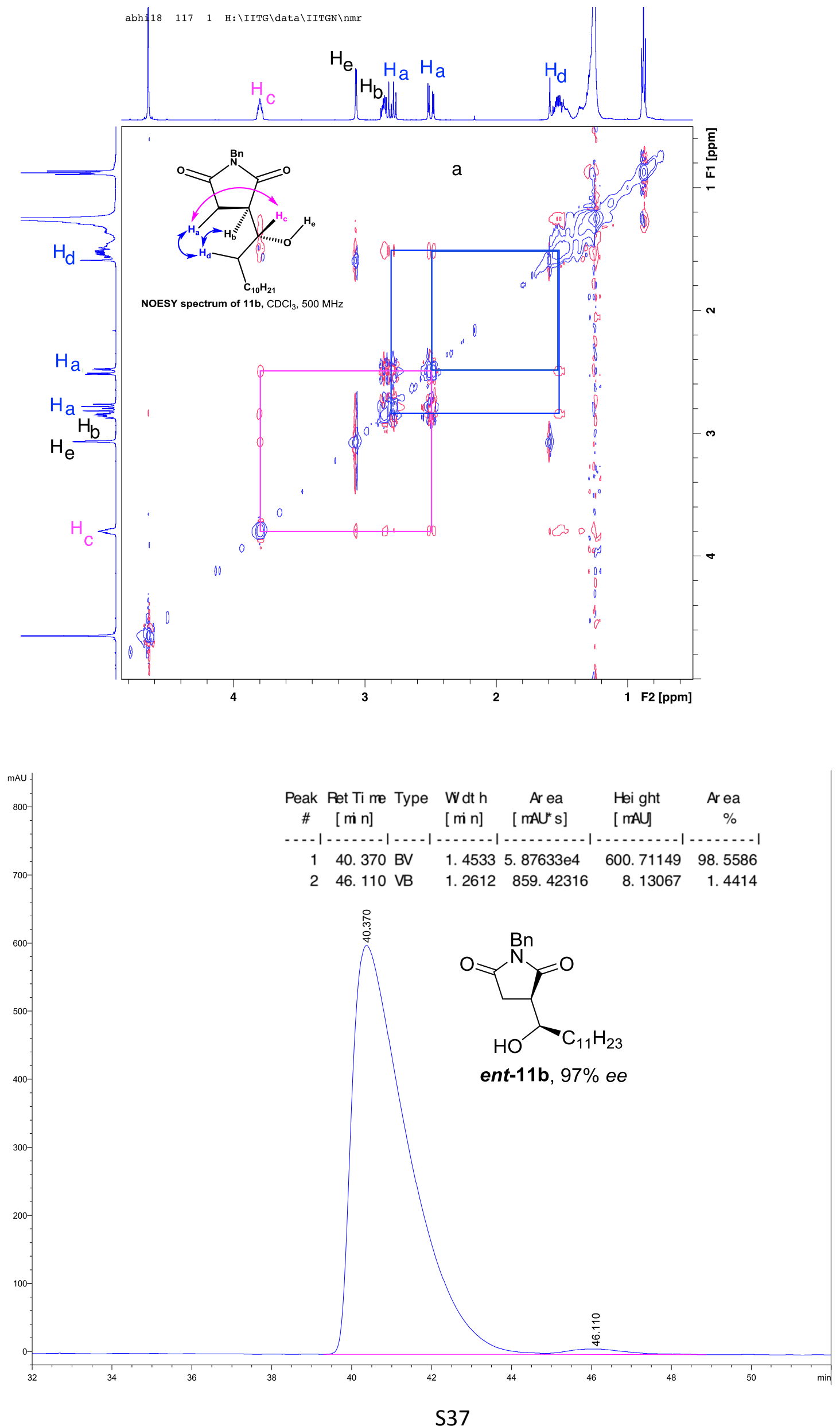


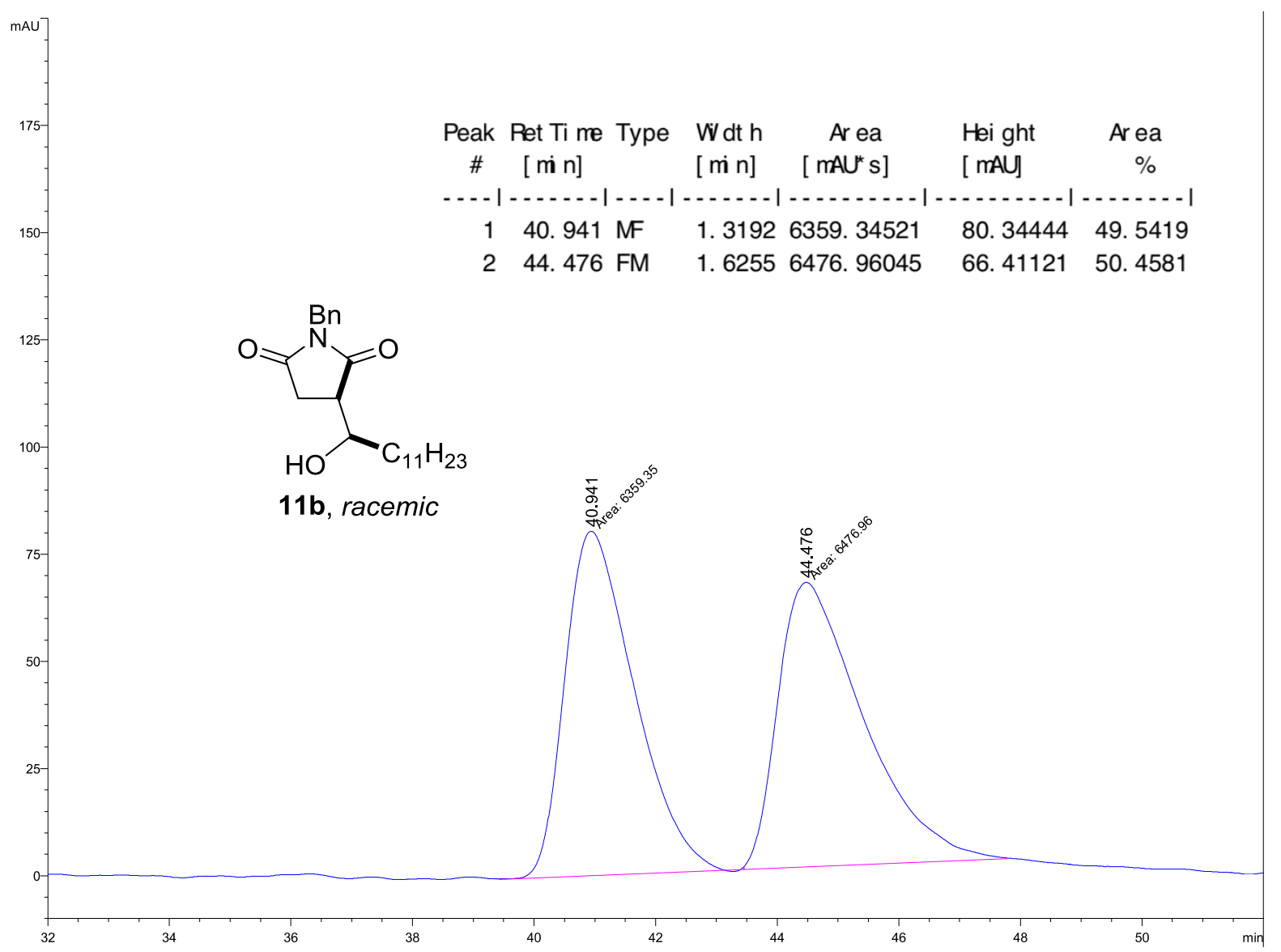

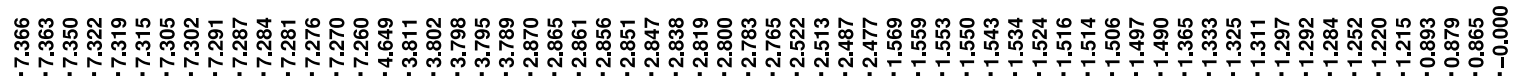

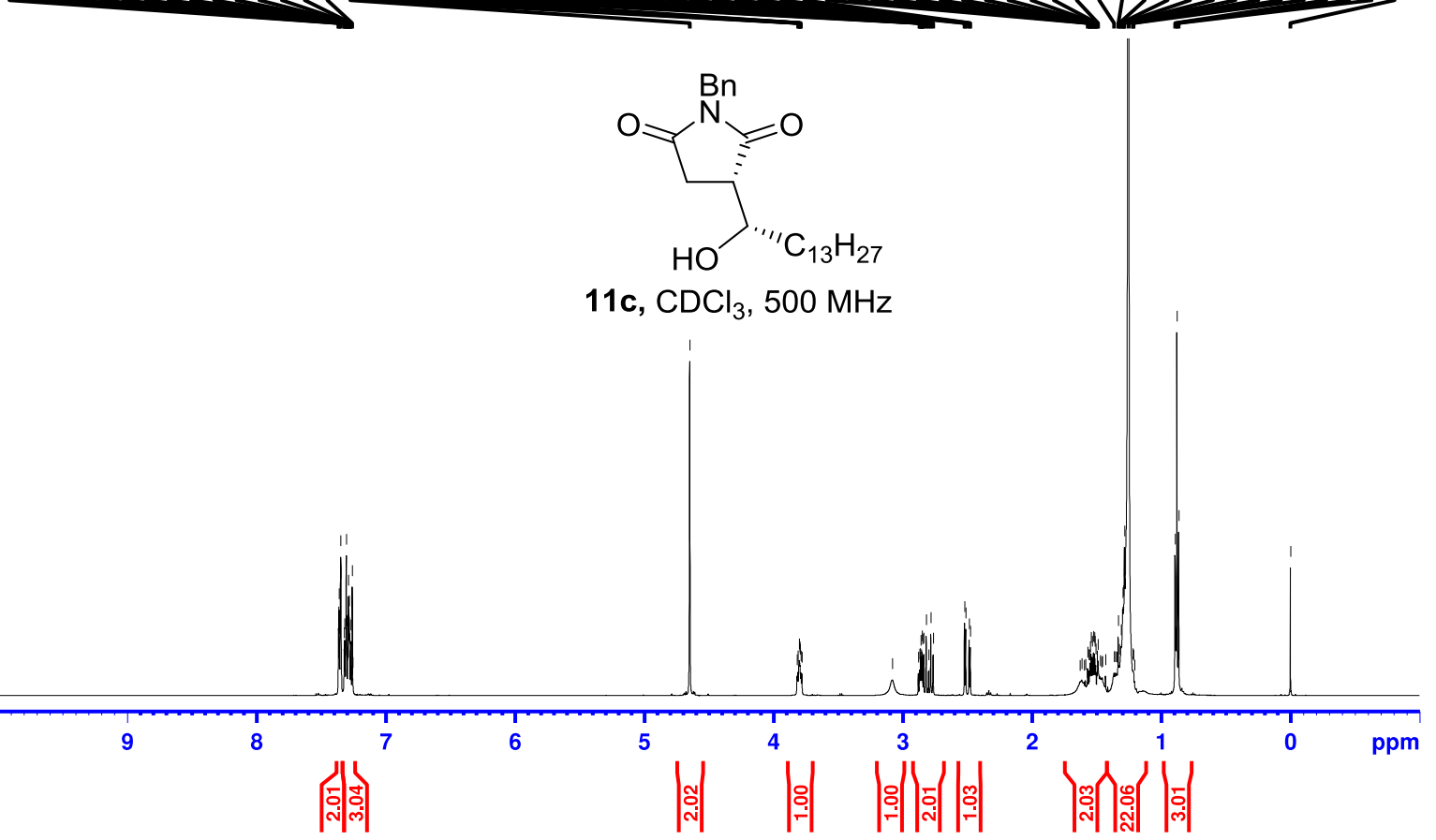



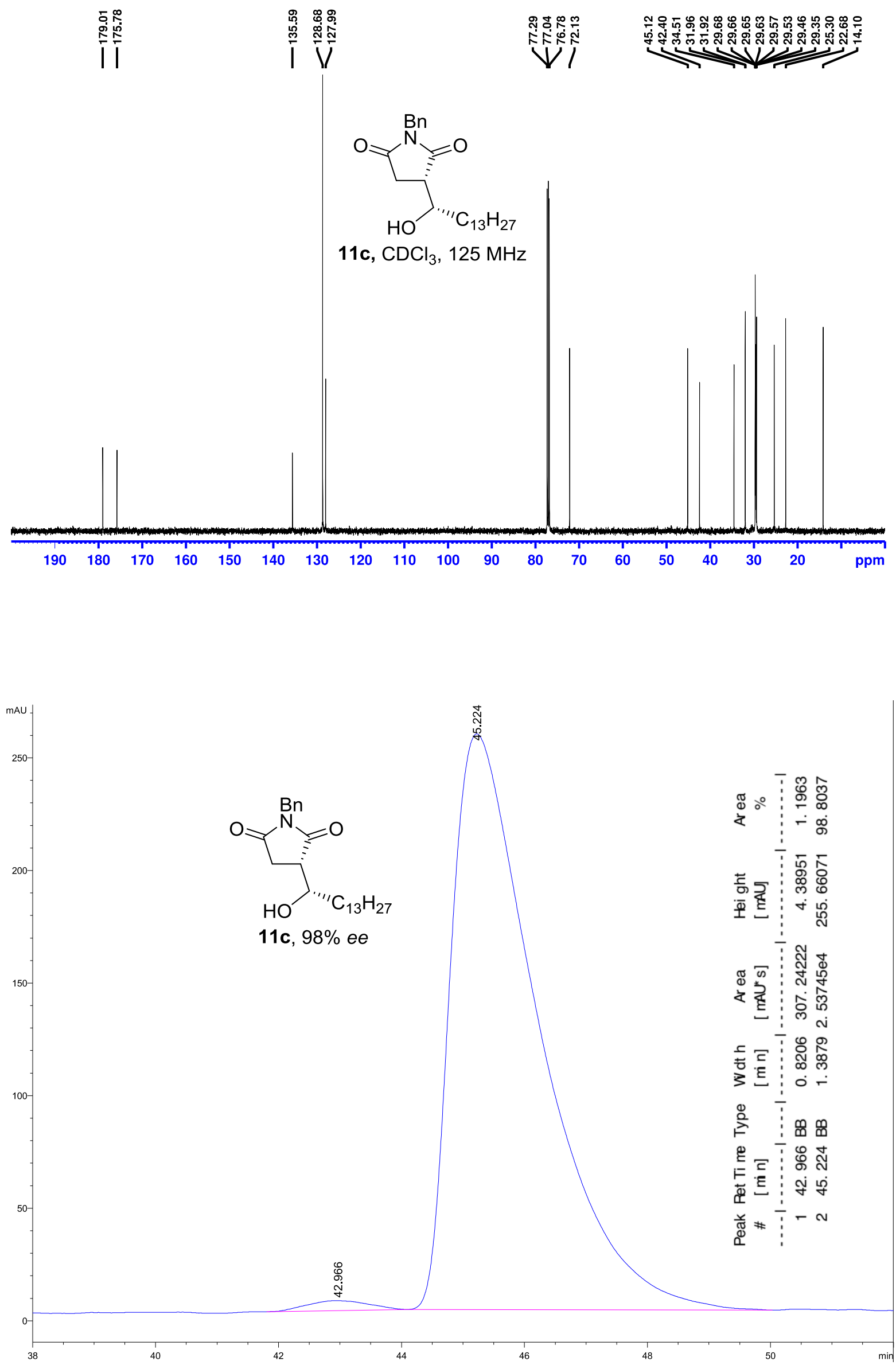

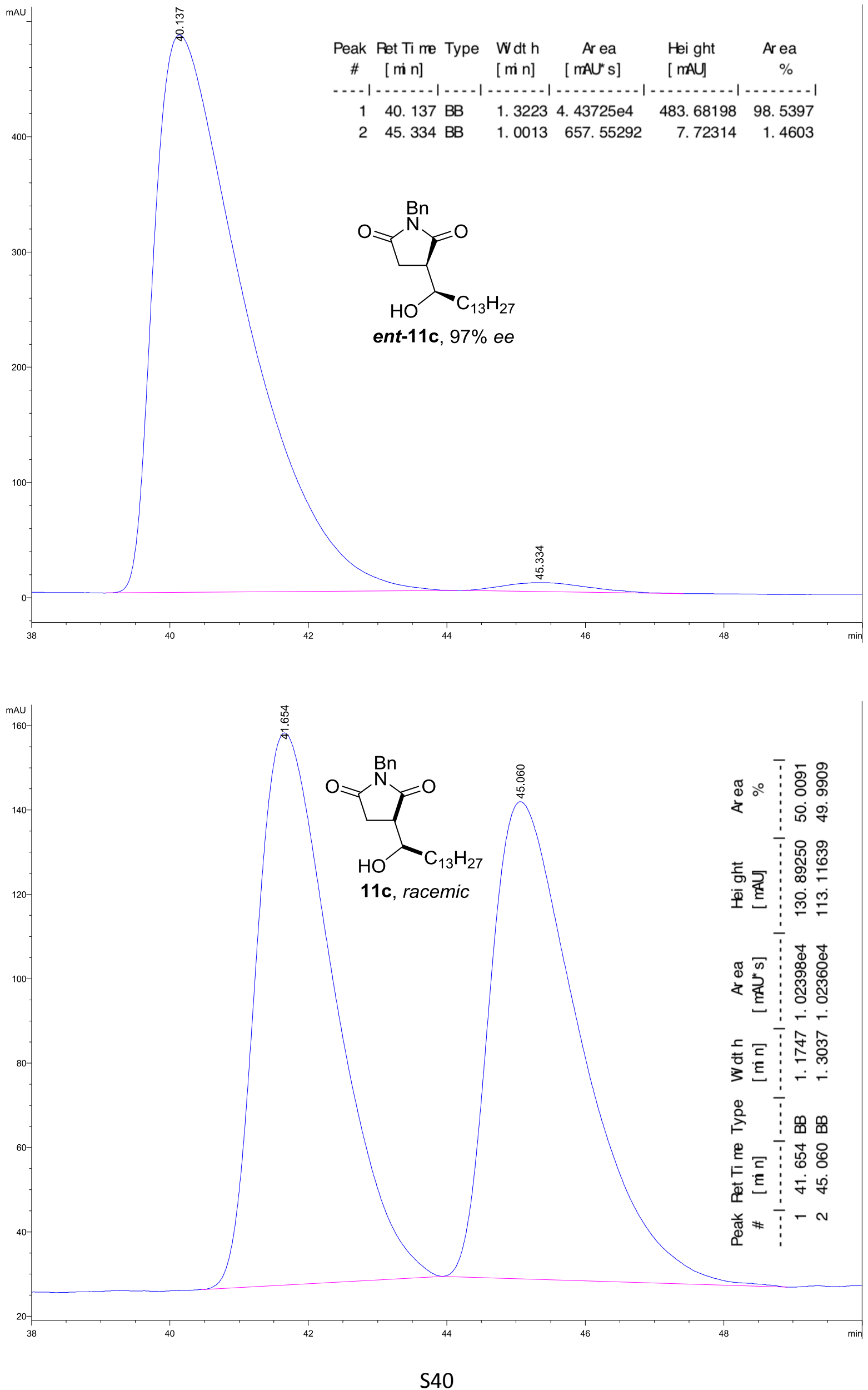


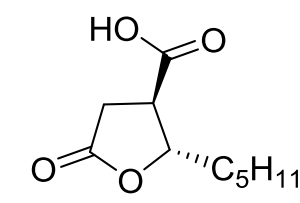

12a, $\mathrm{CDCl}_{3}, 500 \mathrm{MHz}$

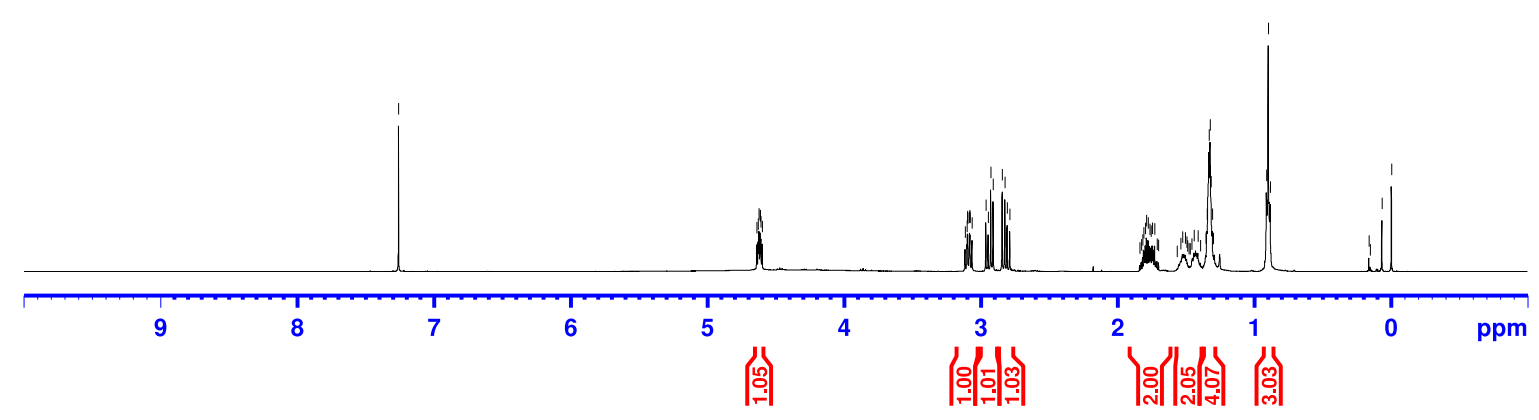

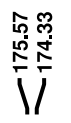
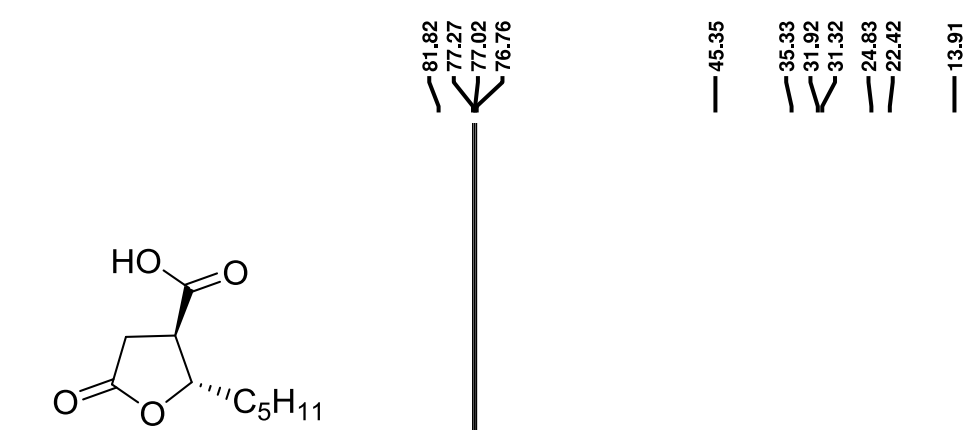

12a, $\mathrm{CDCl}_{3}, 125 \mathrm{MHz}$

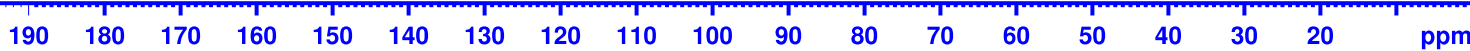




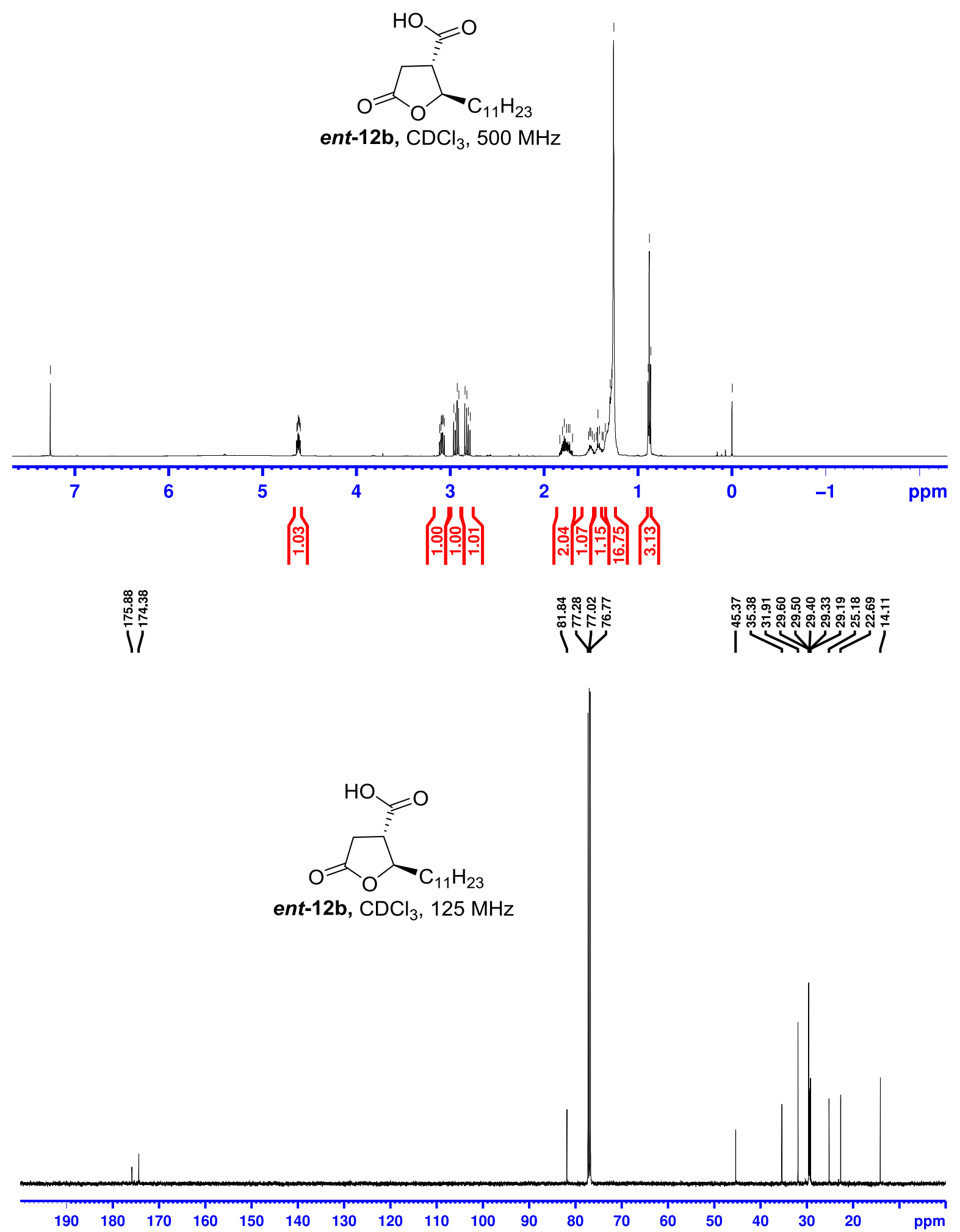



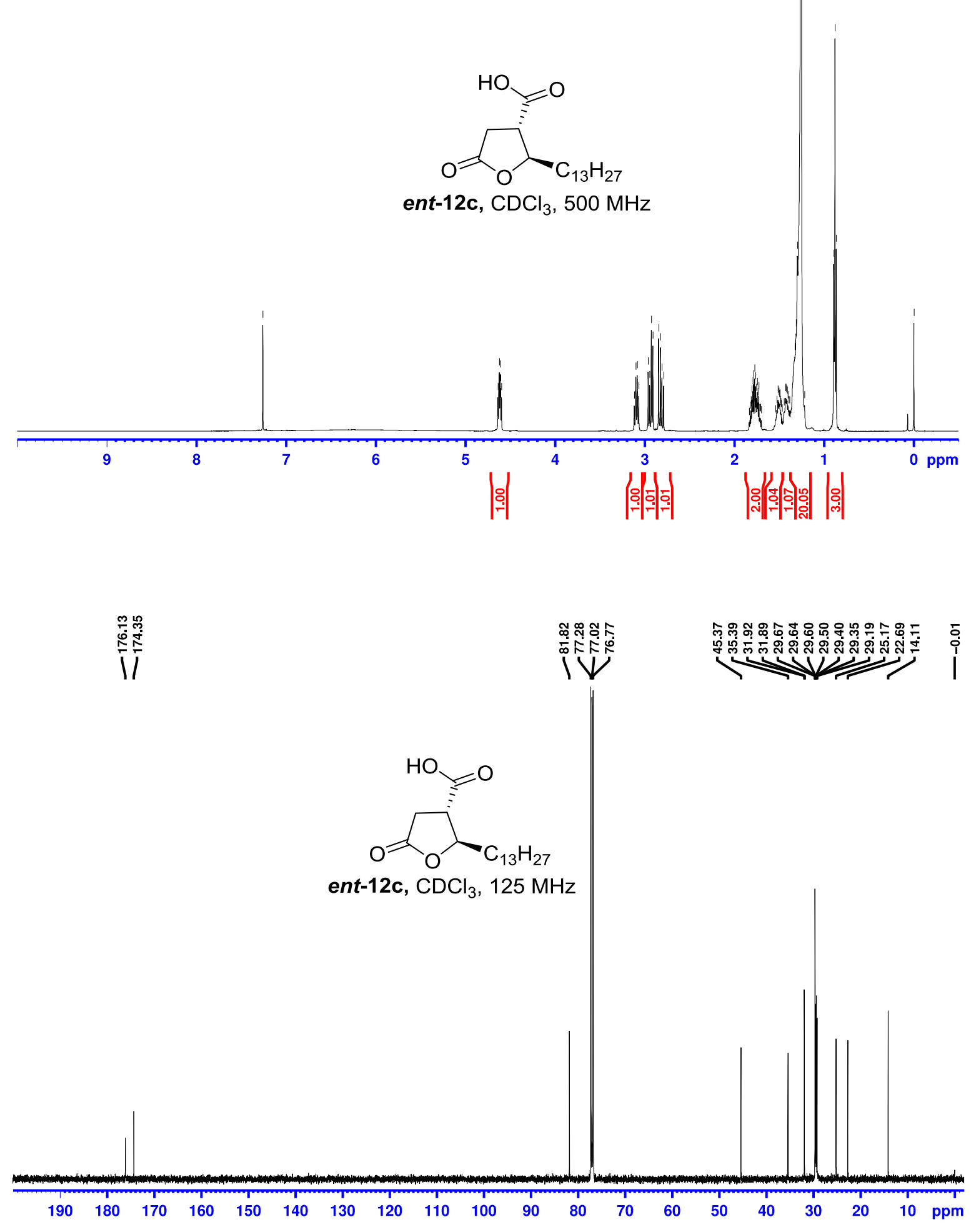


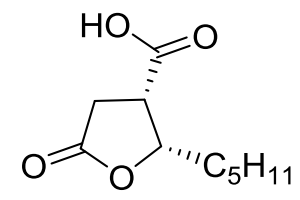

13a, $\mathrm{CDCl}_{3}, 500 \mathrm{MHz}$

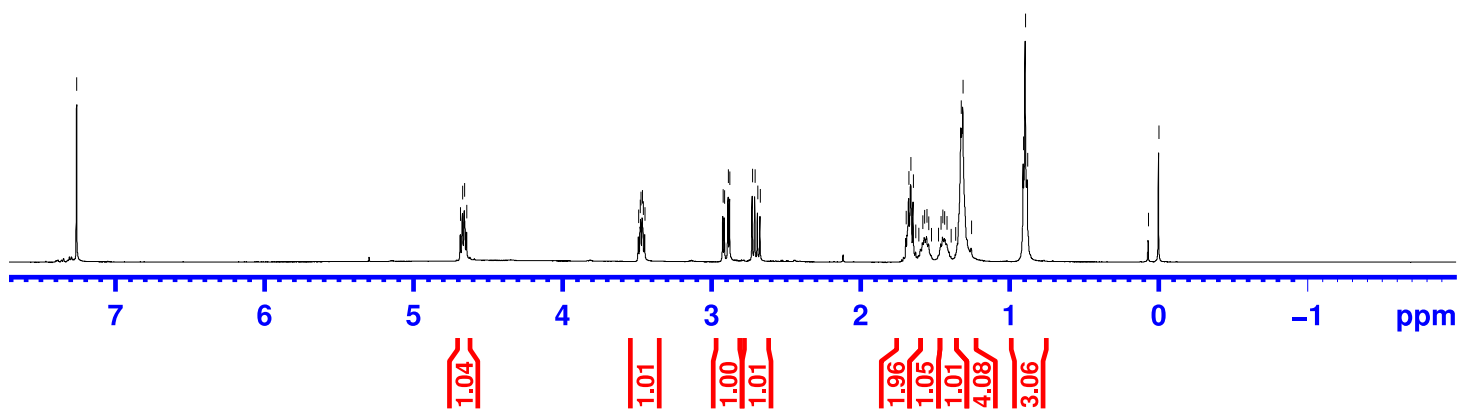

Vִ

|

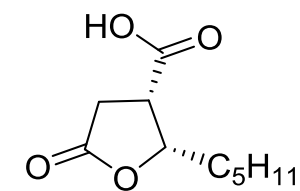

13a, $\mathrm{CDCl}_{3}, 125 \mathrm{MHz}$

190180

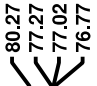

\section{.}



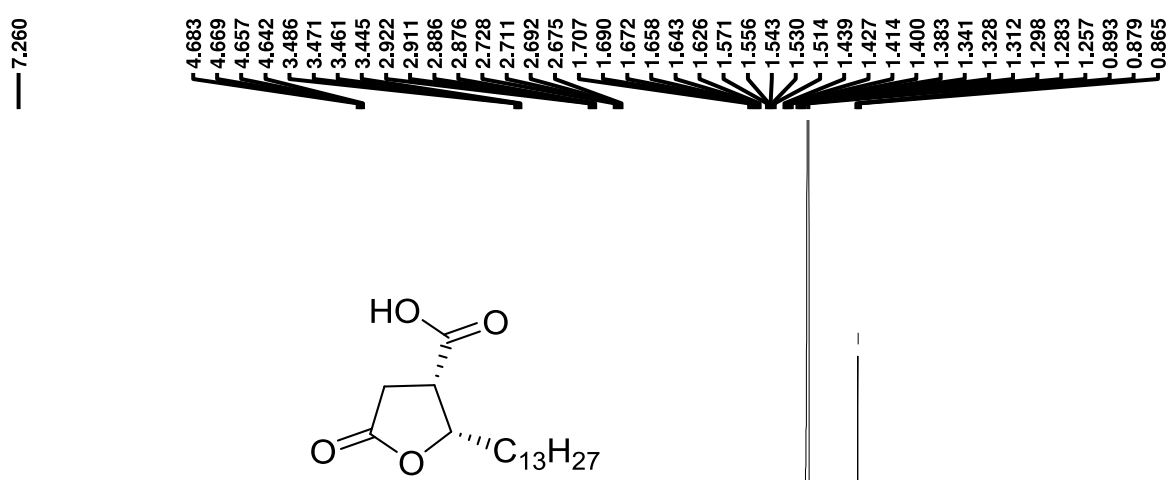

13c, $\mathrm{CDCl}_{3}, 500 \mathrm{MHz}$

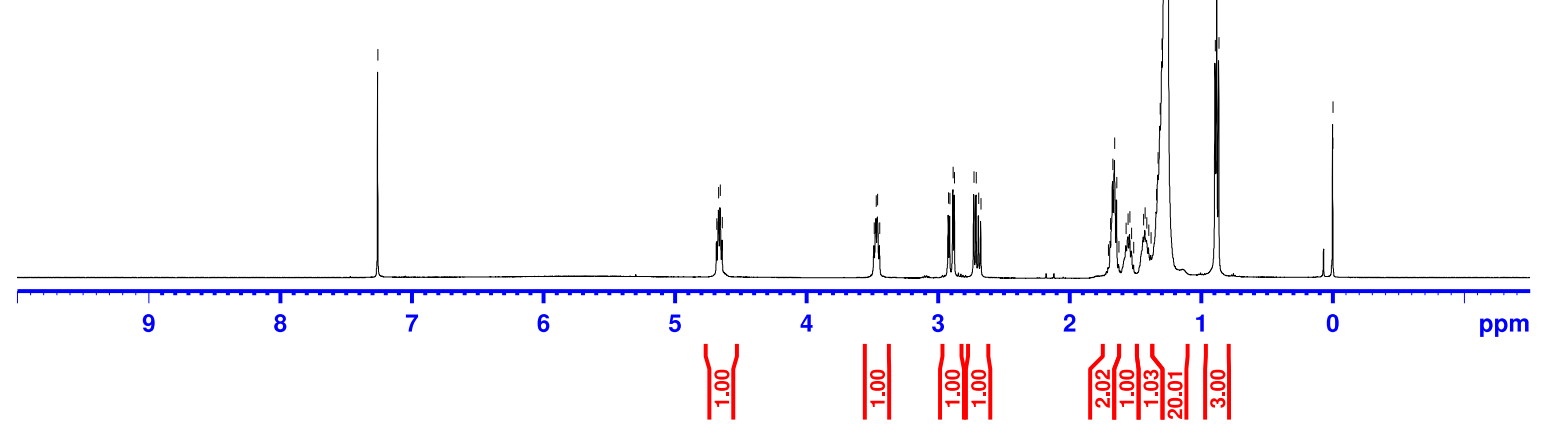

高
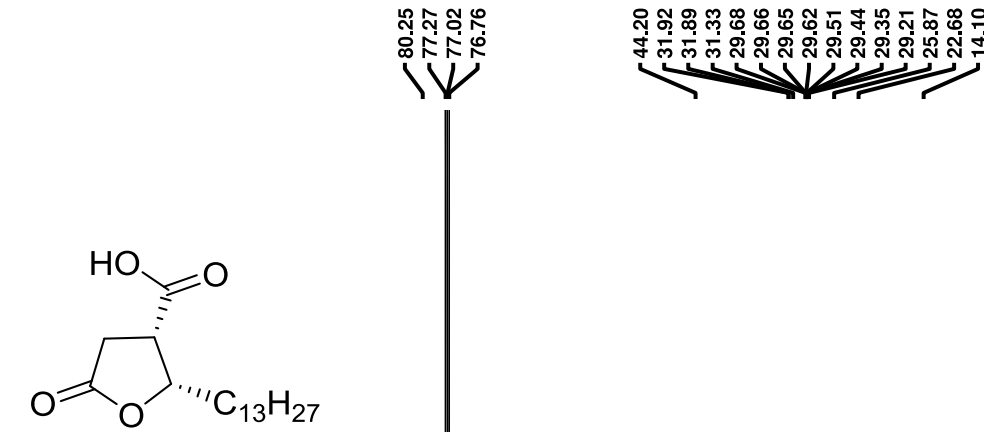

13c, $\mathrm{CDCl}_{3}, 125 \mathrm{MHz}$

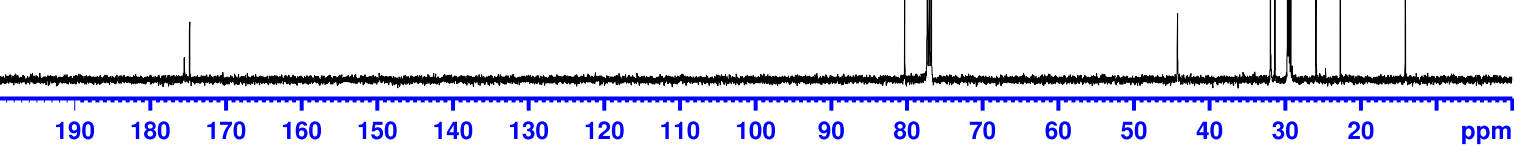




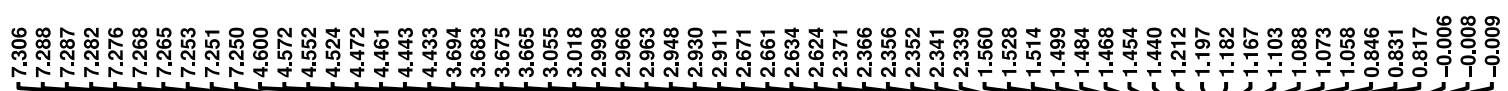

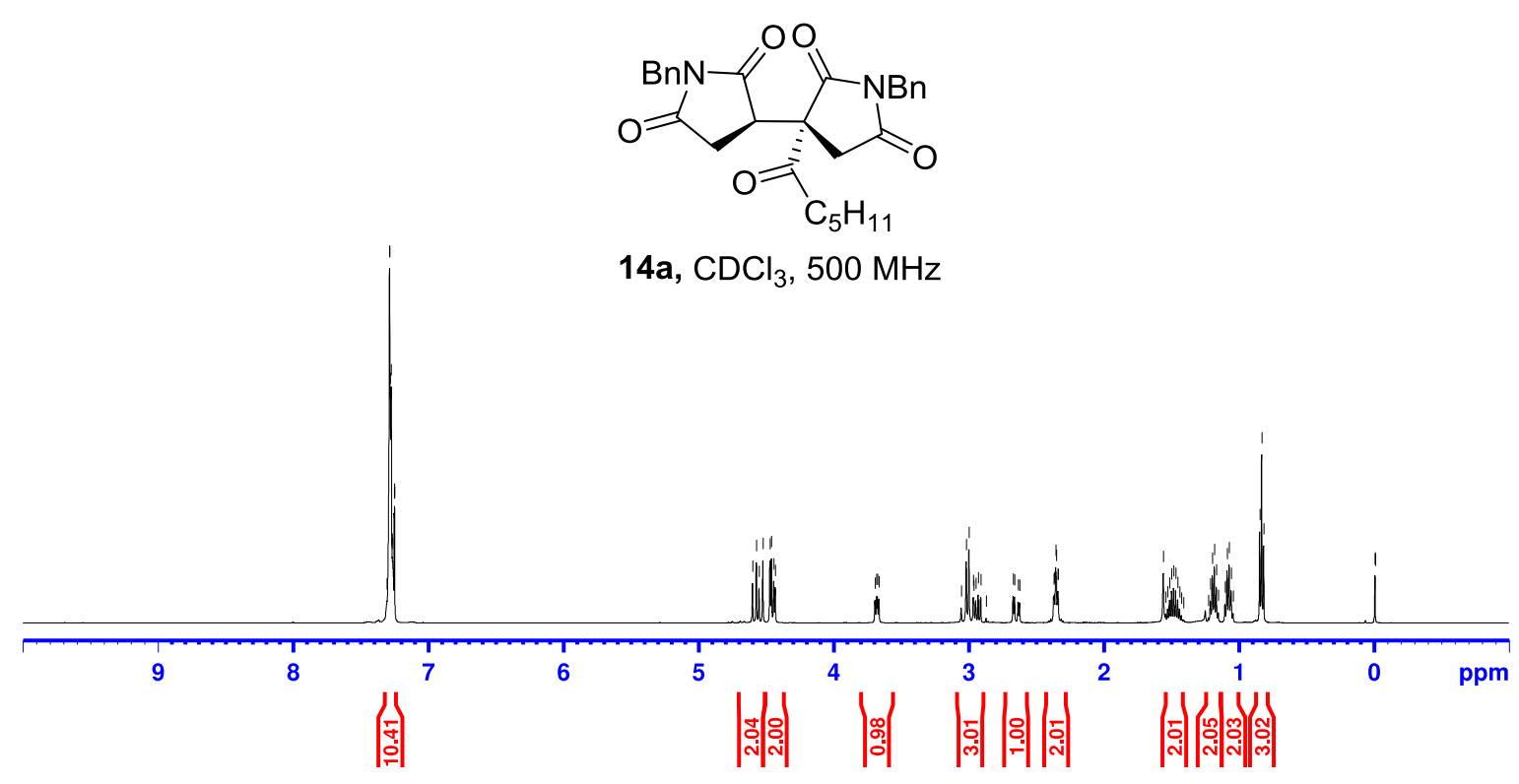

I

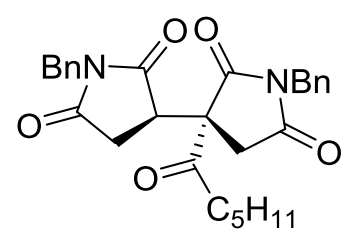

14a, $\mathrm{CDCl}_{3}, 125 \mathrm{MHz}$

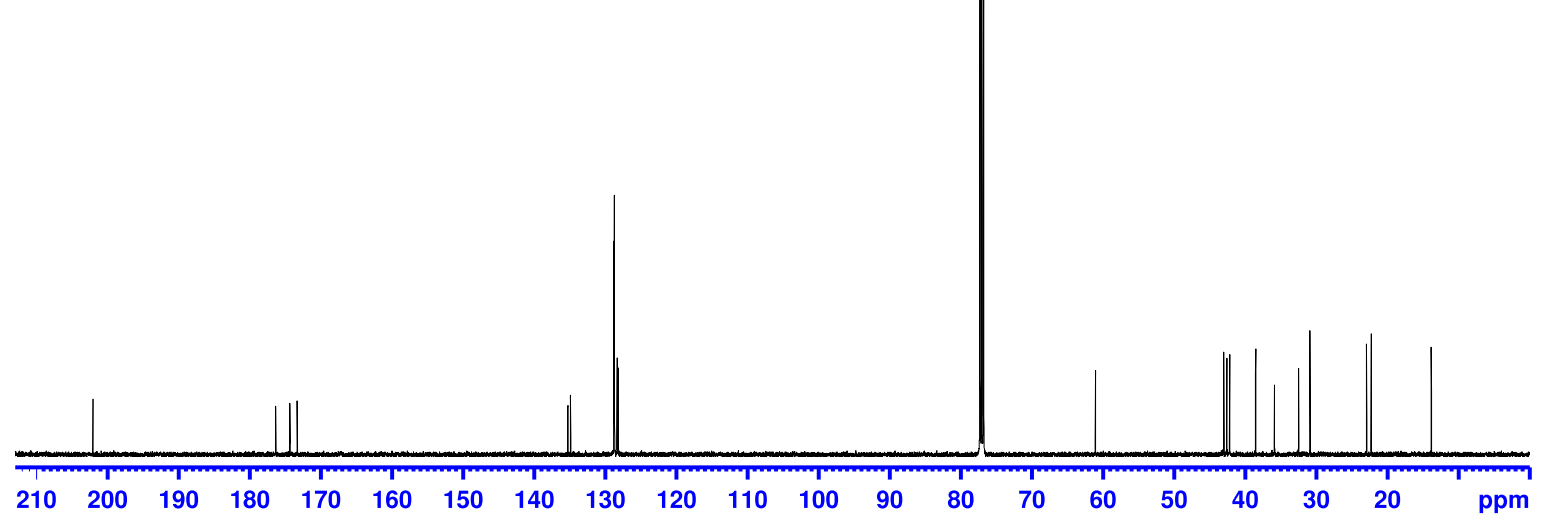



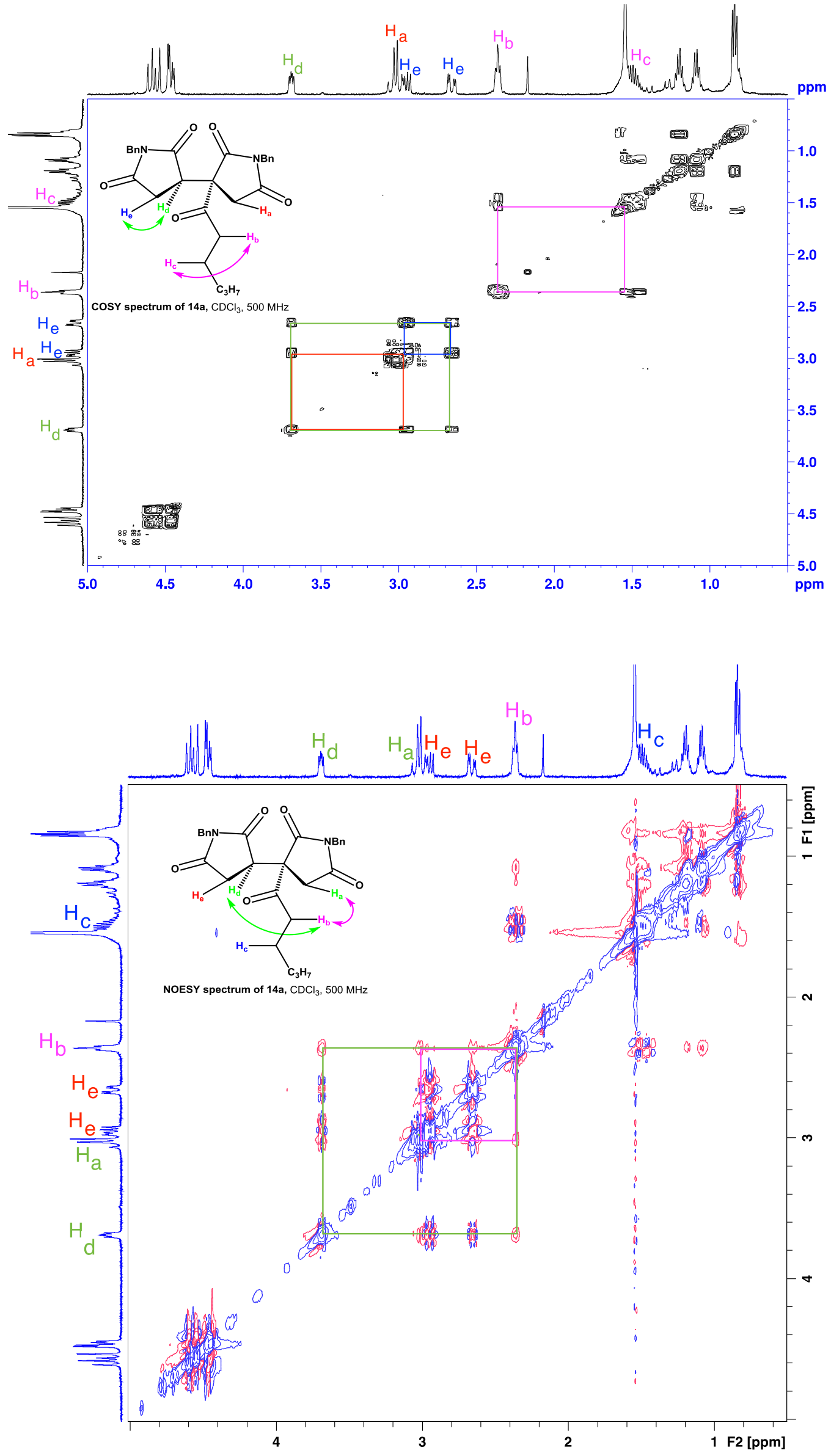


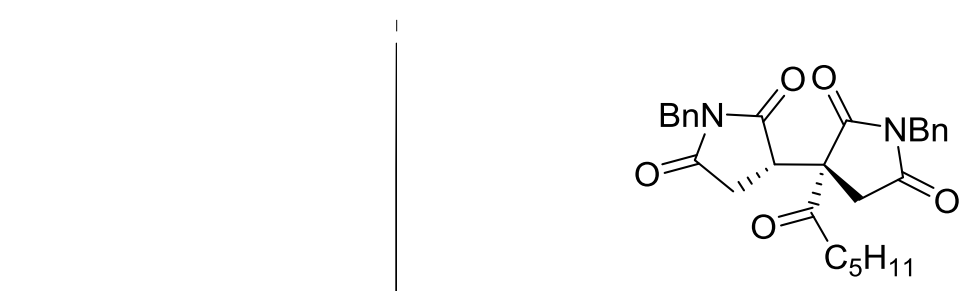

14b, $\mathrm{CDCl}_{3}, 500 \mathrm{MHz}$

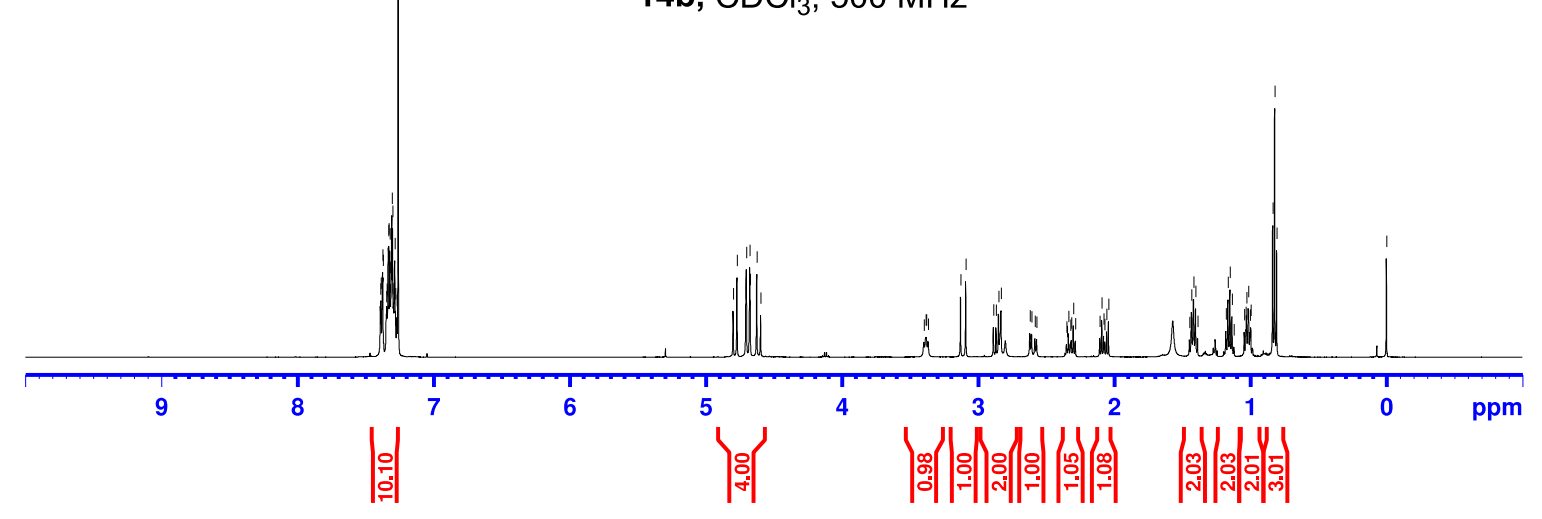

|⿱乛龰๊

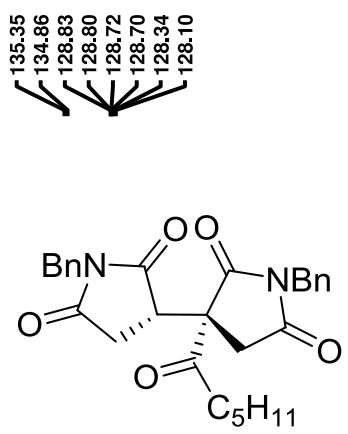

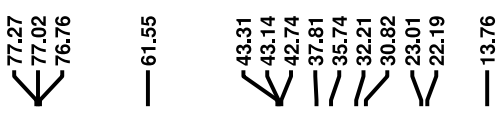

14b, $\mathrm{CDCl}_{3}, 125 \mathrm{MHz}$

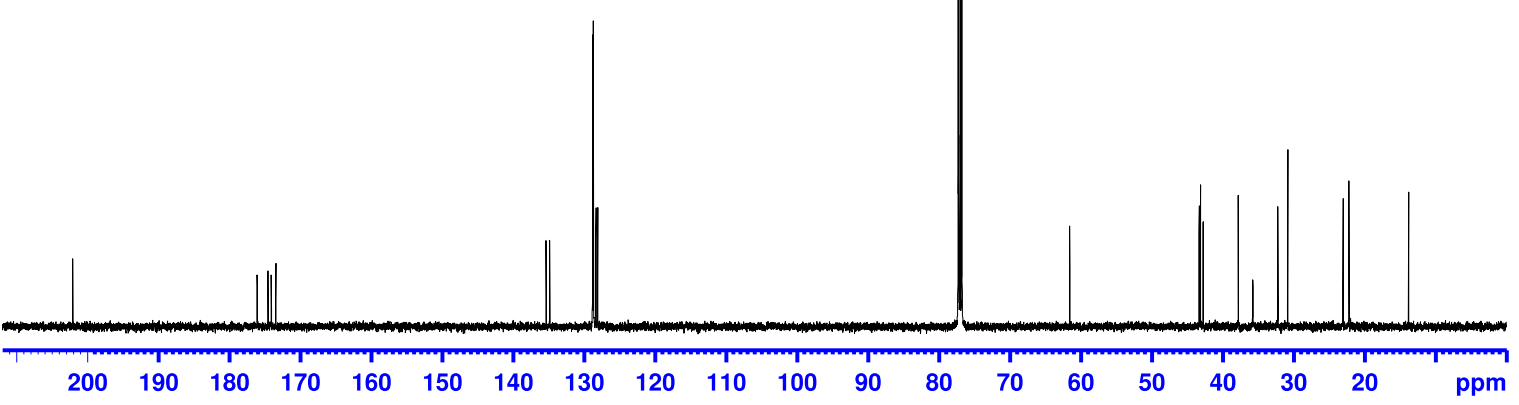




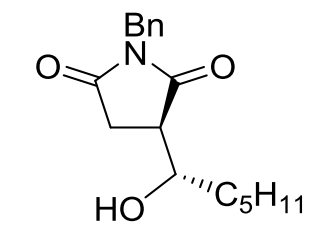

15a, $\mathrm{CDCl}_{3}, 500 \mathrm{MHz}$
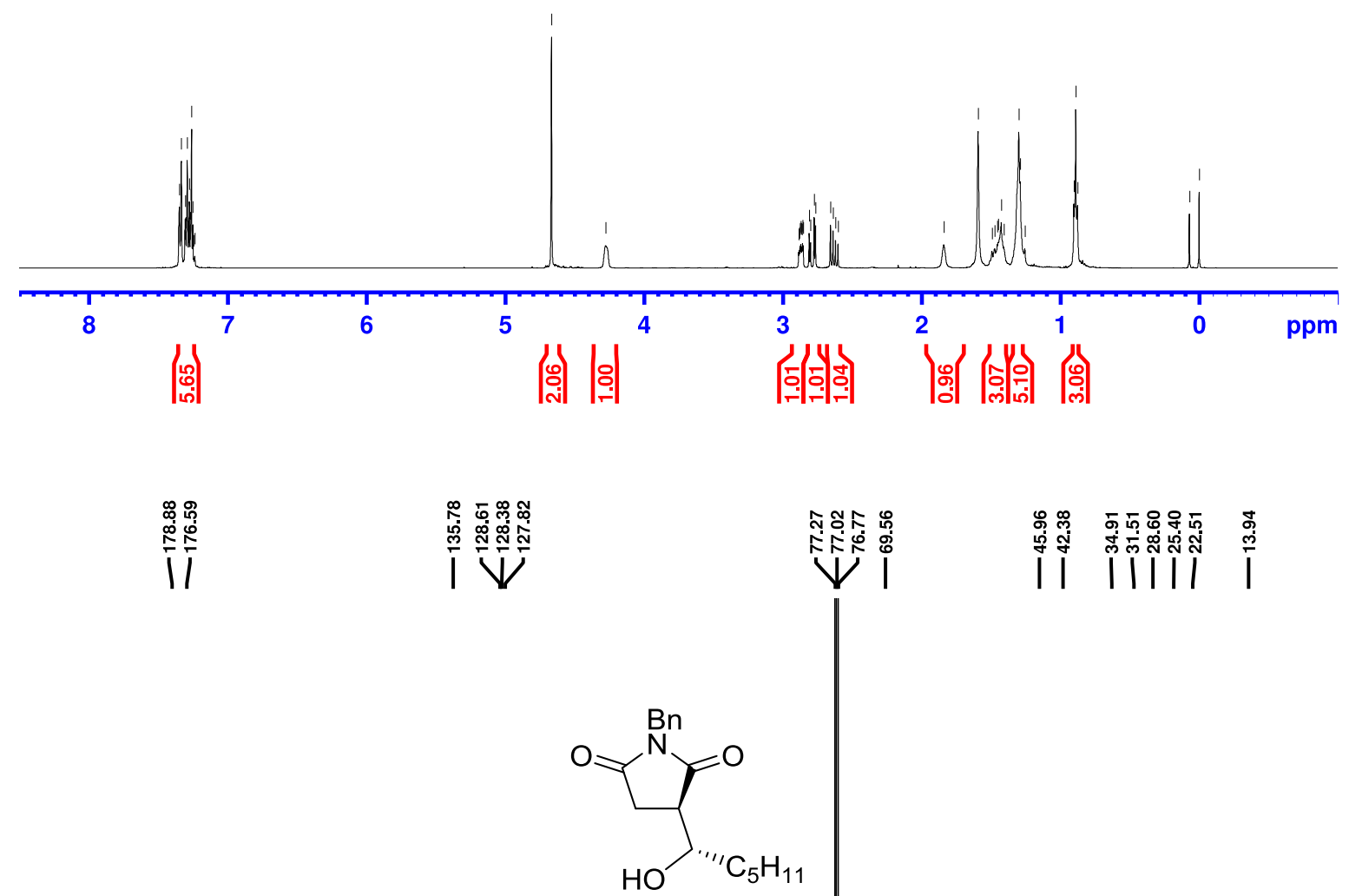

15a, $\mathrm{CDCl}_{3}, 125 \mathrm{MHz}$

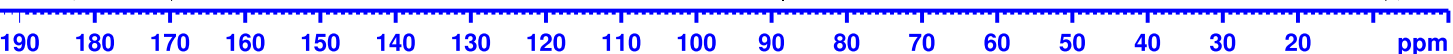



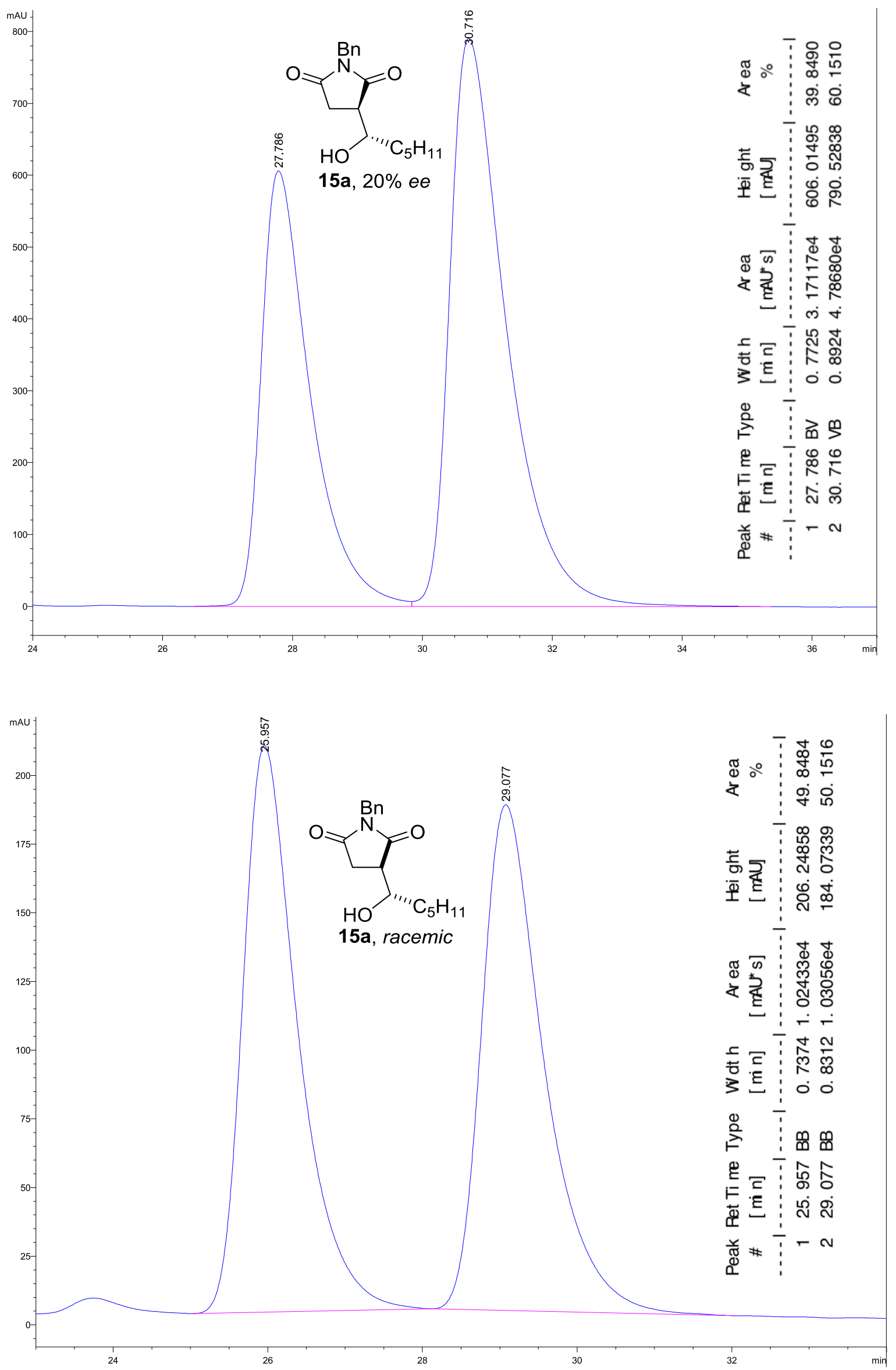


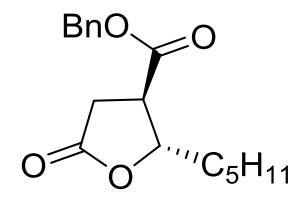

16a, $\mathrm{CDCl}_{3}, 500 \mathrm{MHz}$
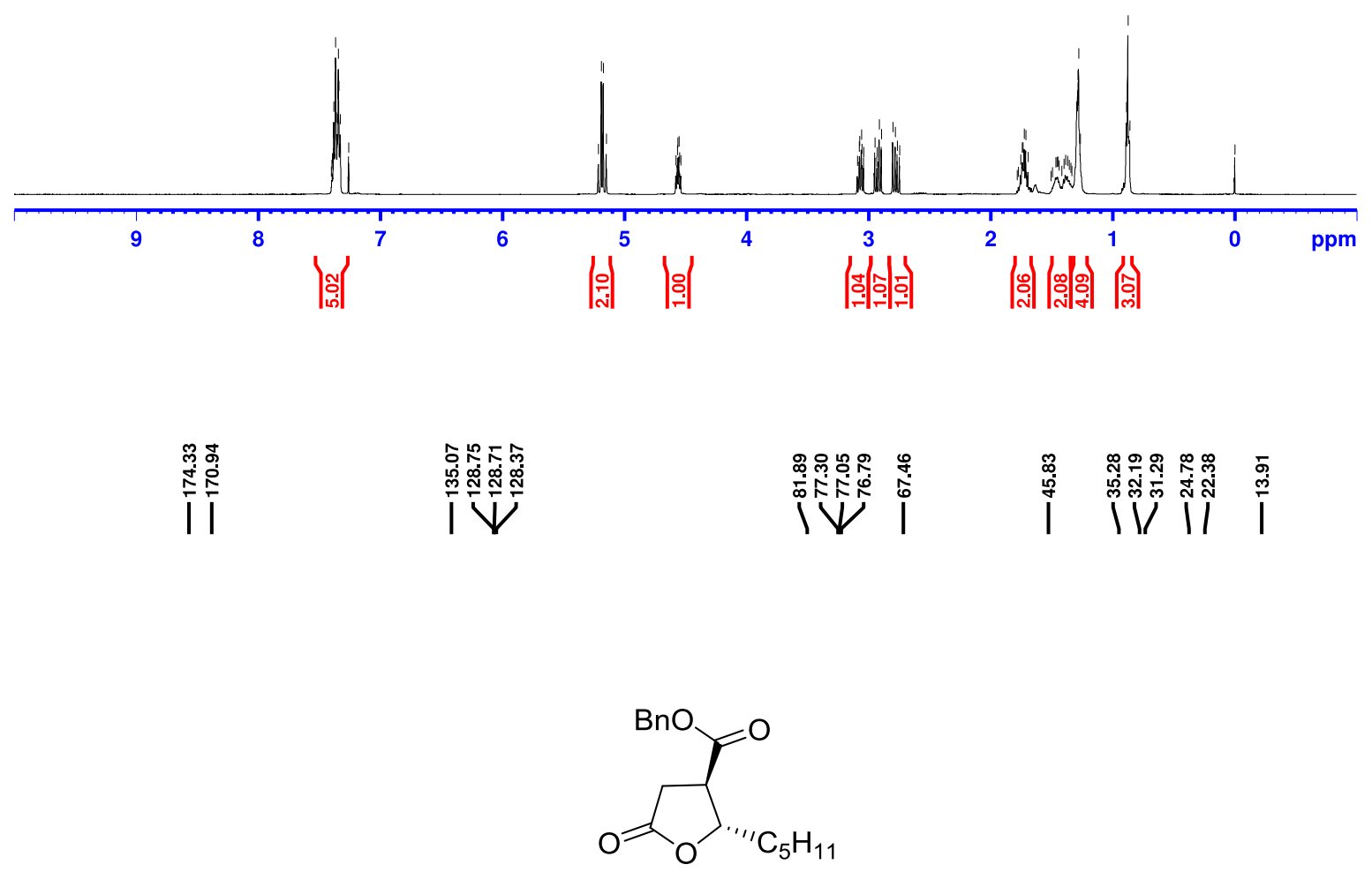

16a, $\mathrm{CDCl}_{3}, 125 \mathrm{MHz}$

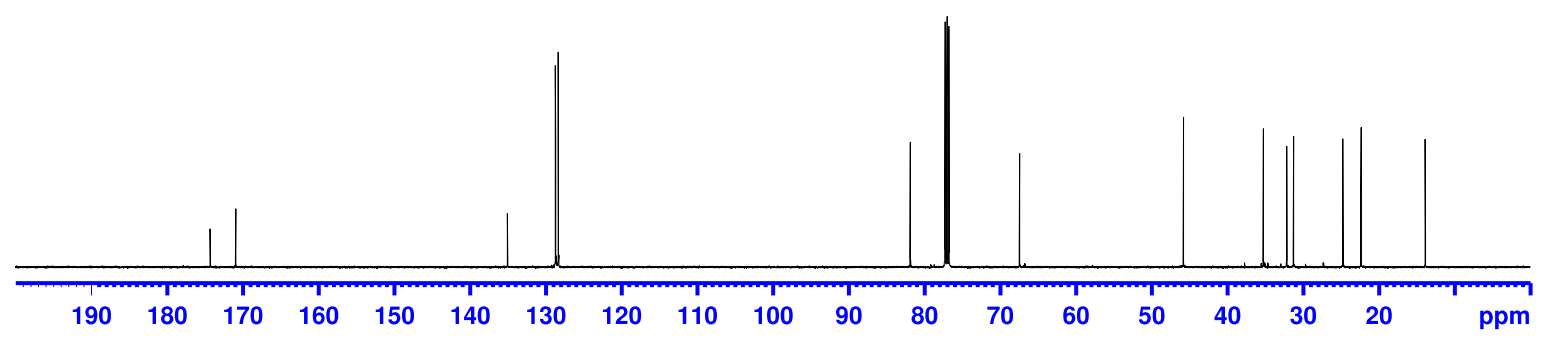



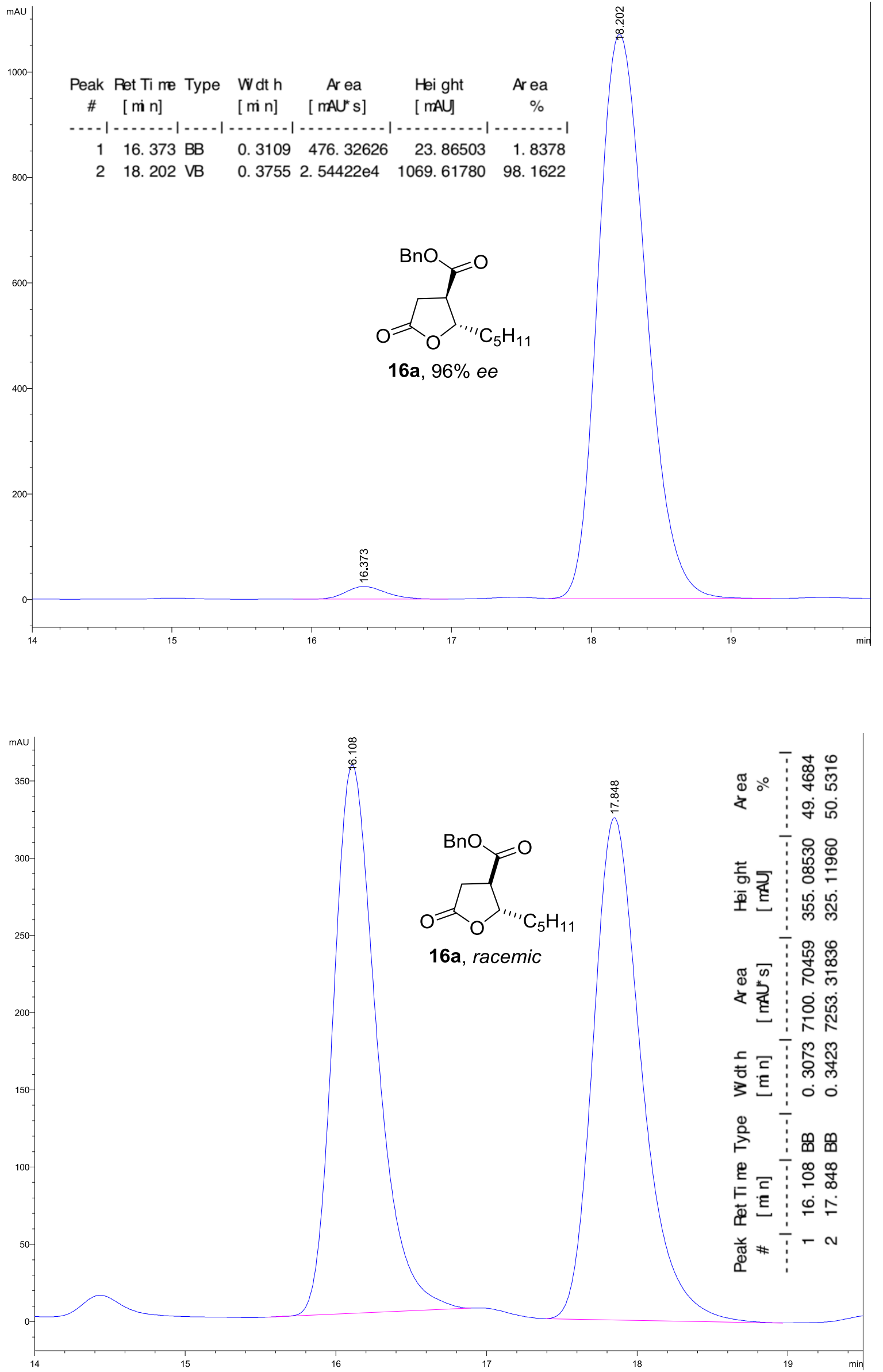


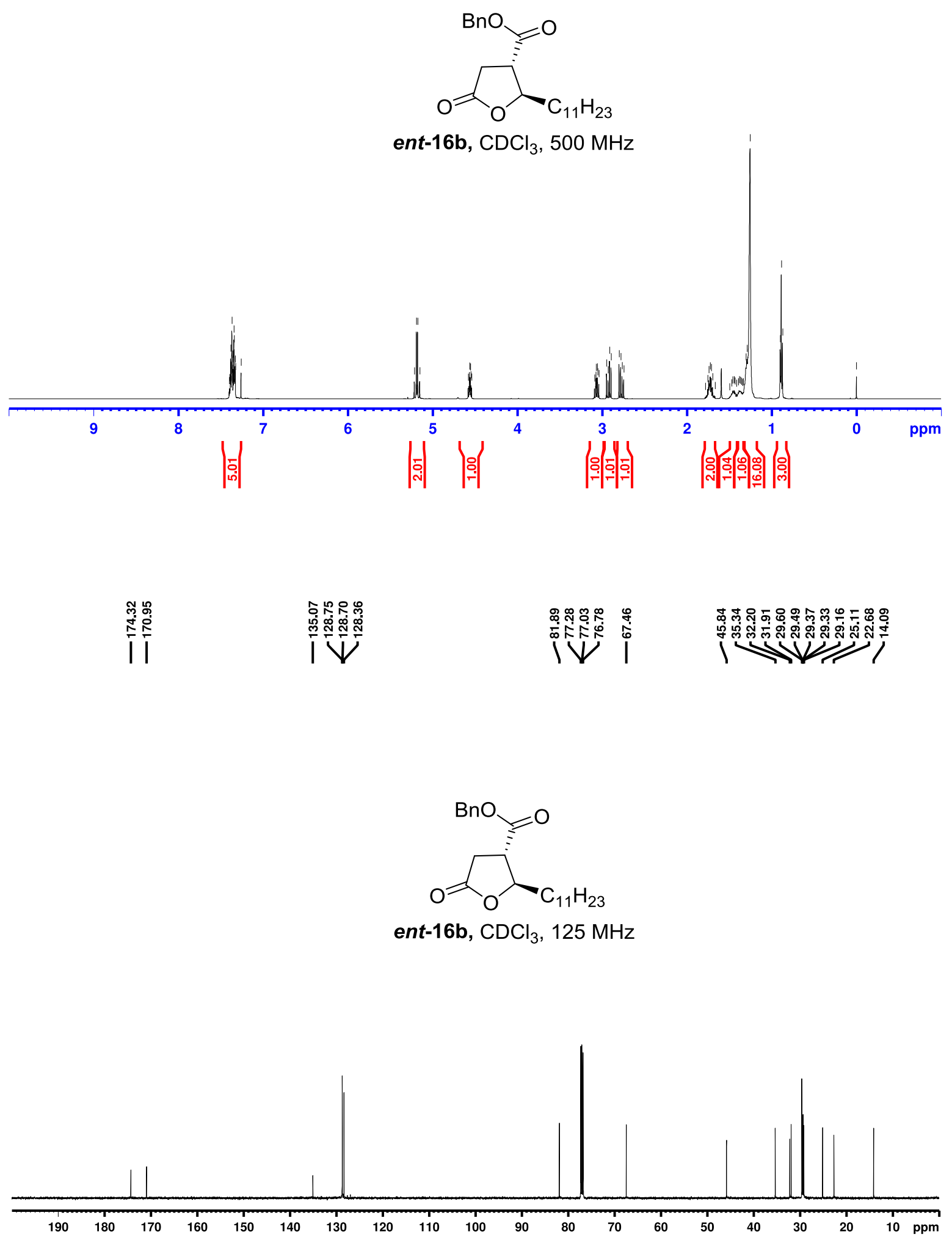



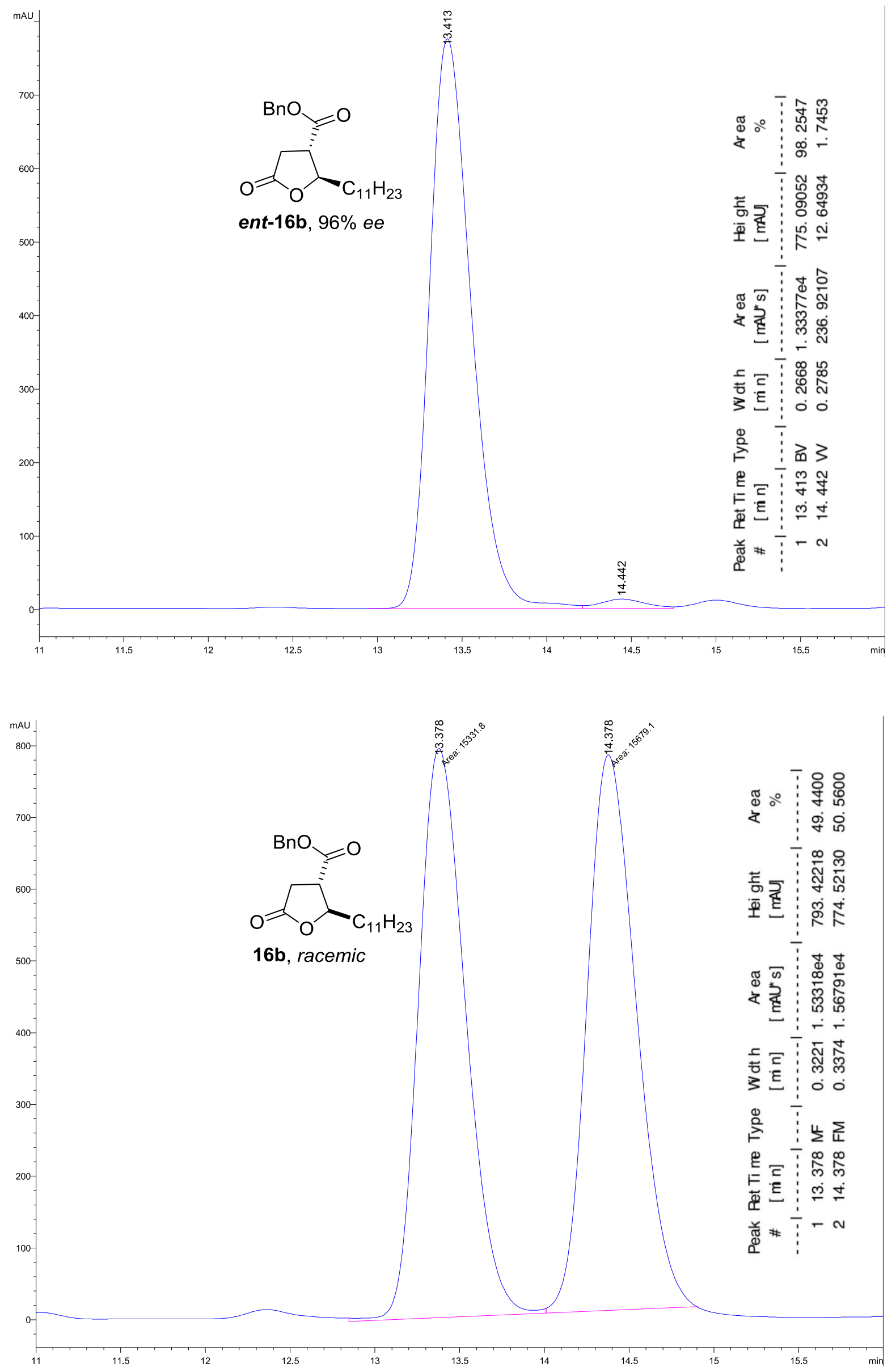

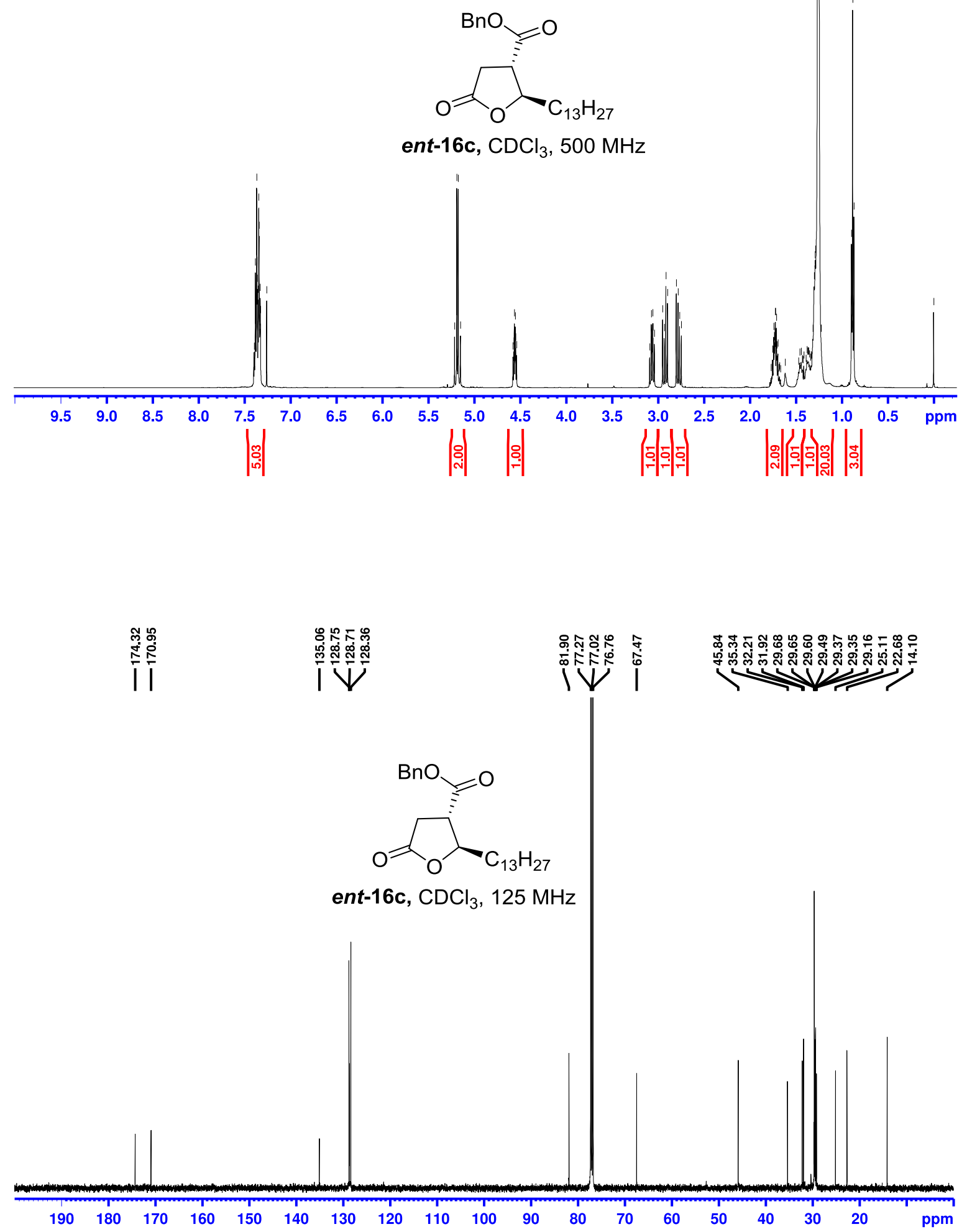

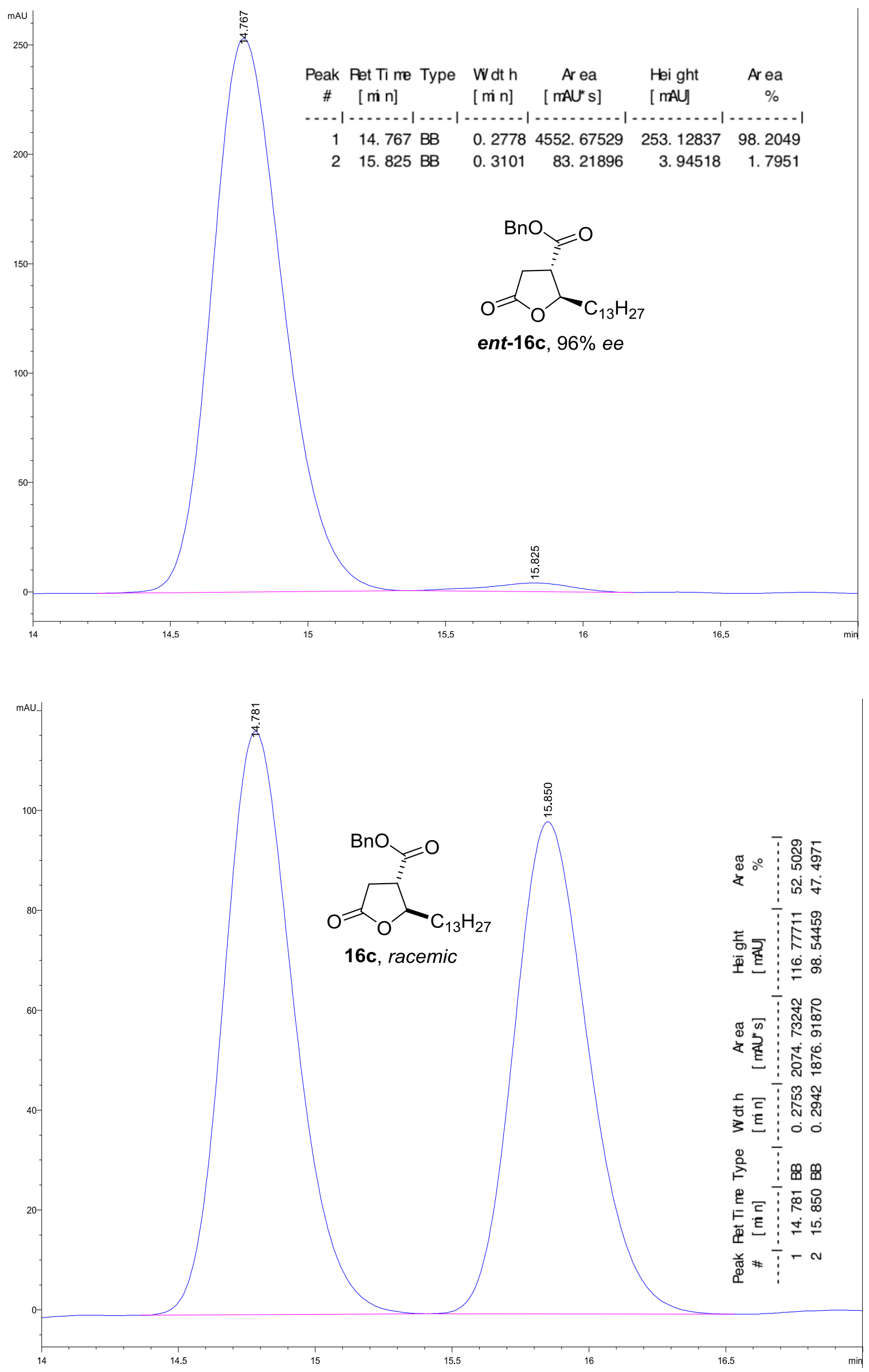


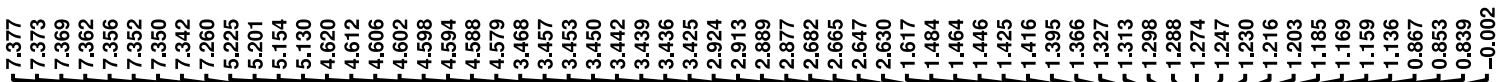

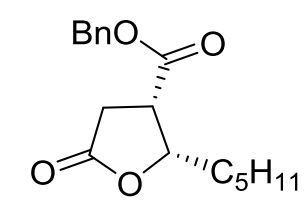

17a, $\mathrm{CDCl}_{3}, 500 \mathrm{MHz}$
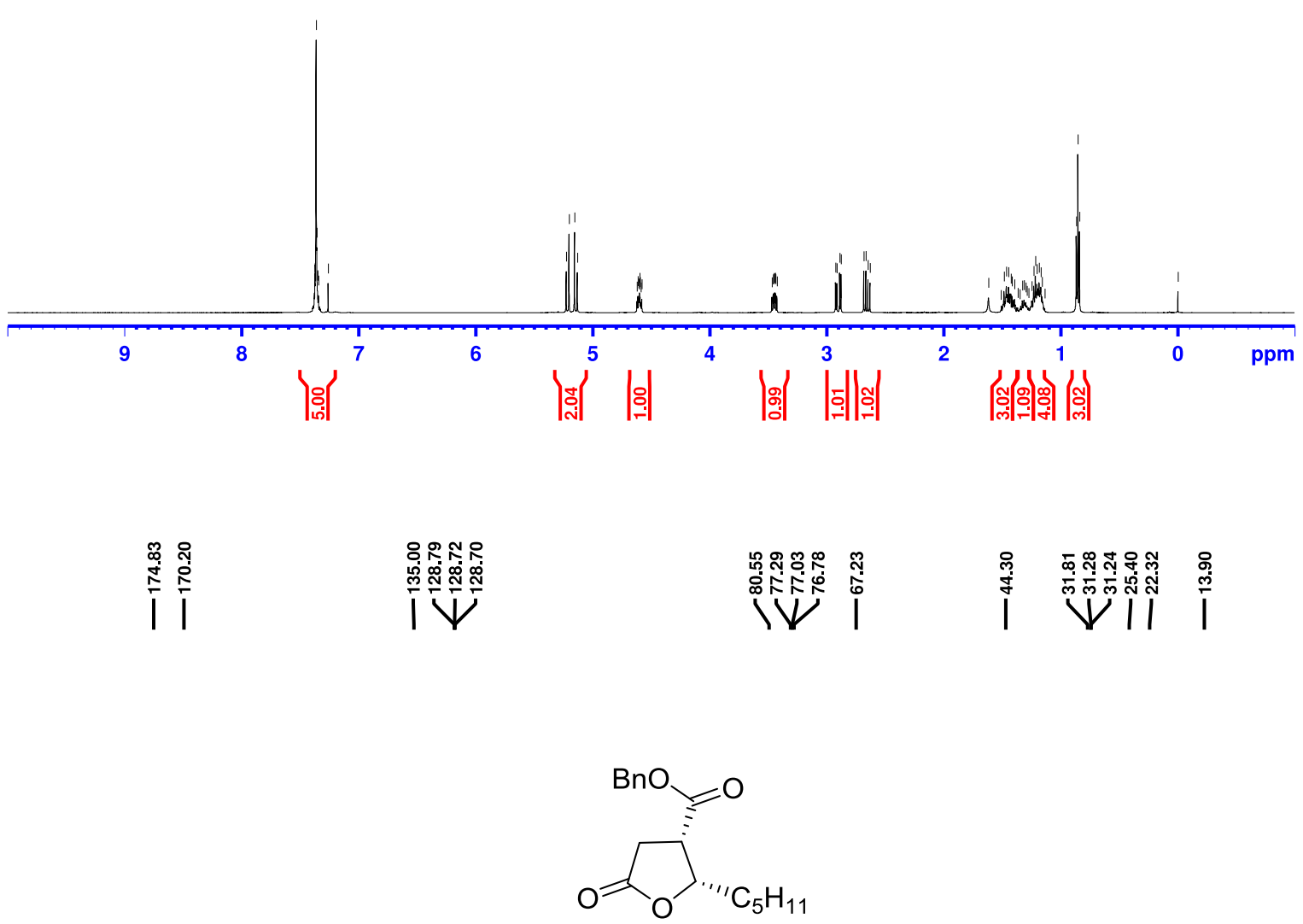

17a, $\mathrm{CDCl}_{3}, 125 \mathrm{MHz}$

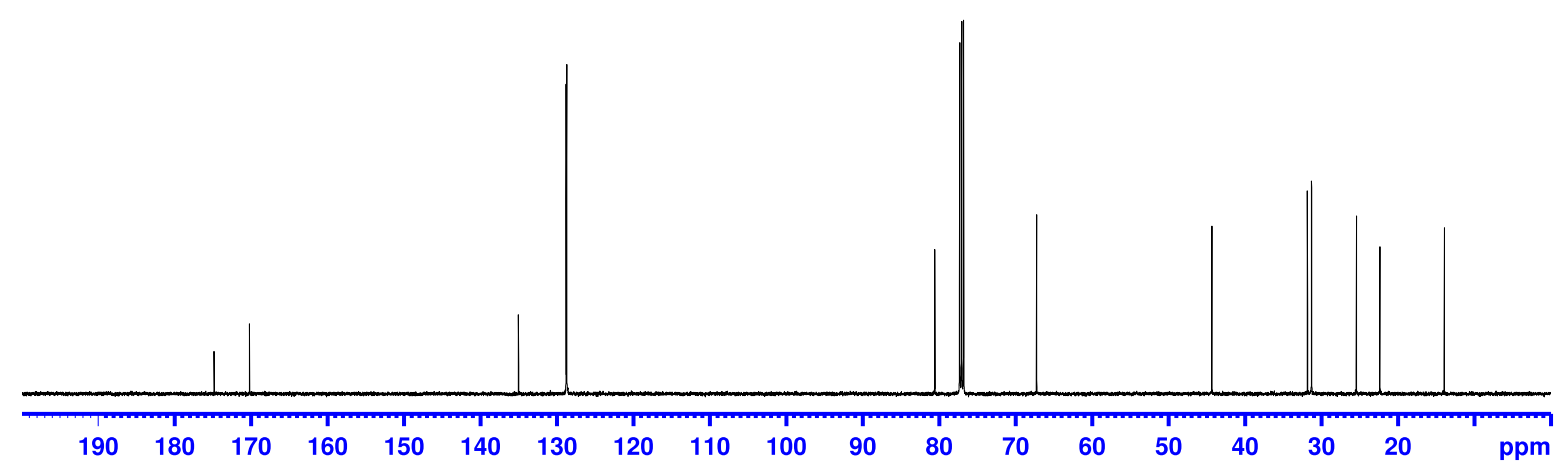



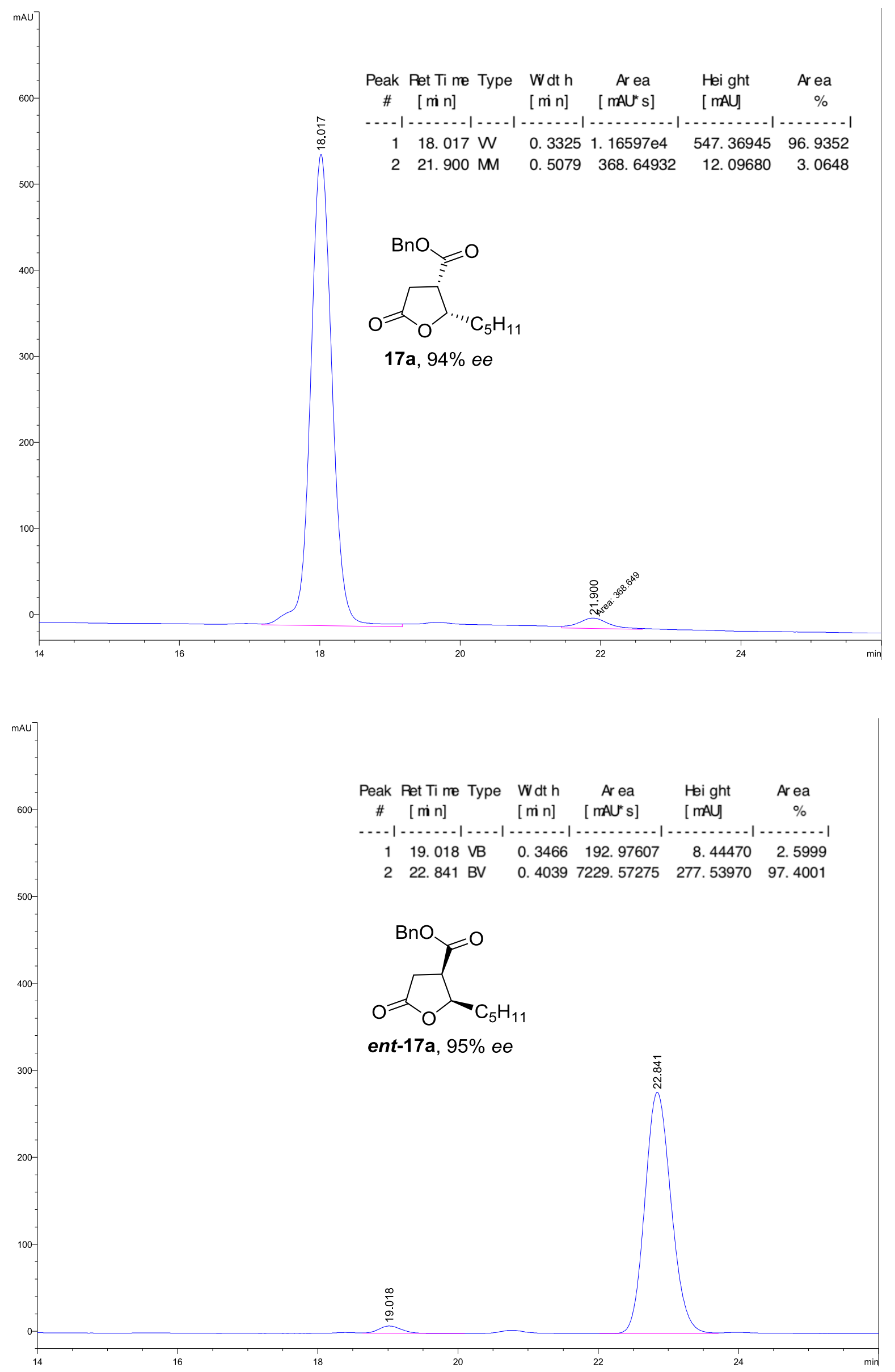


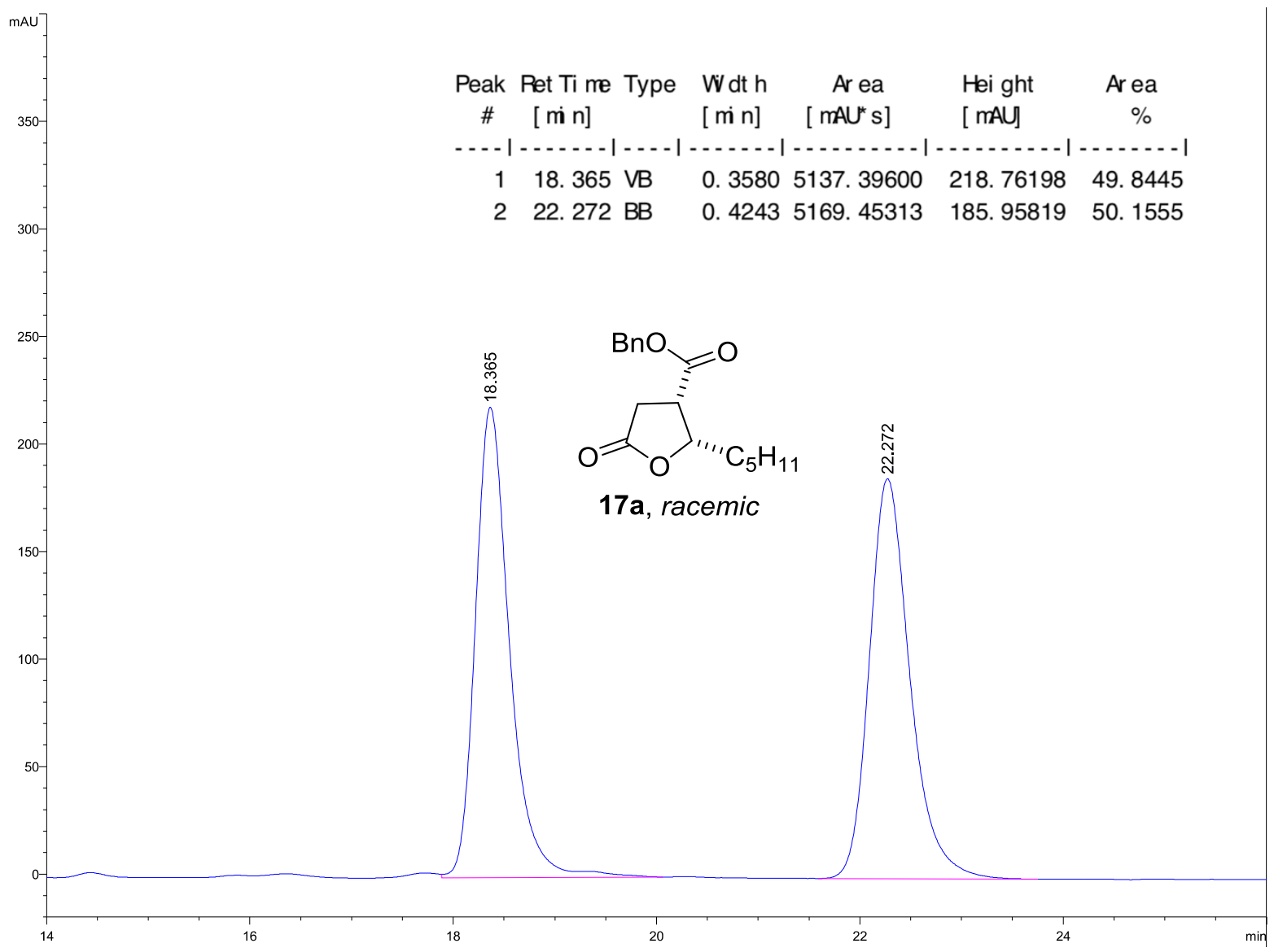

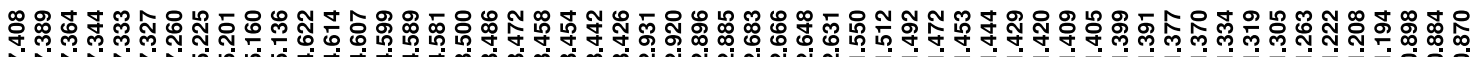
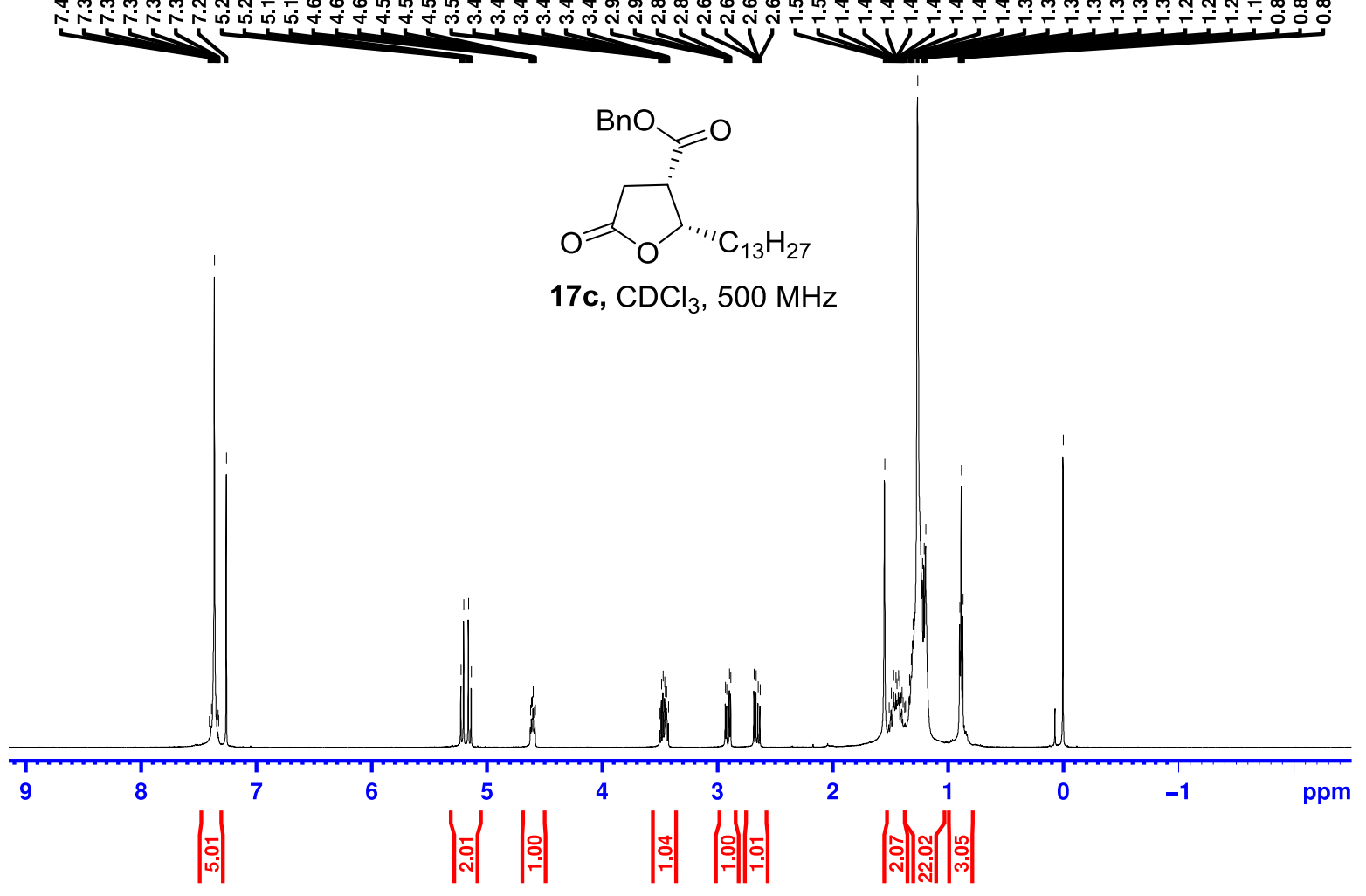

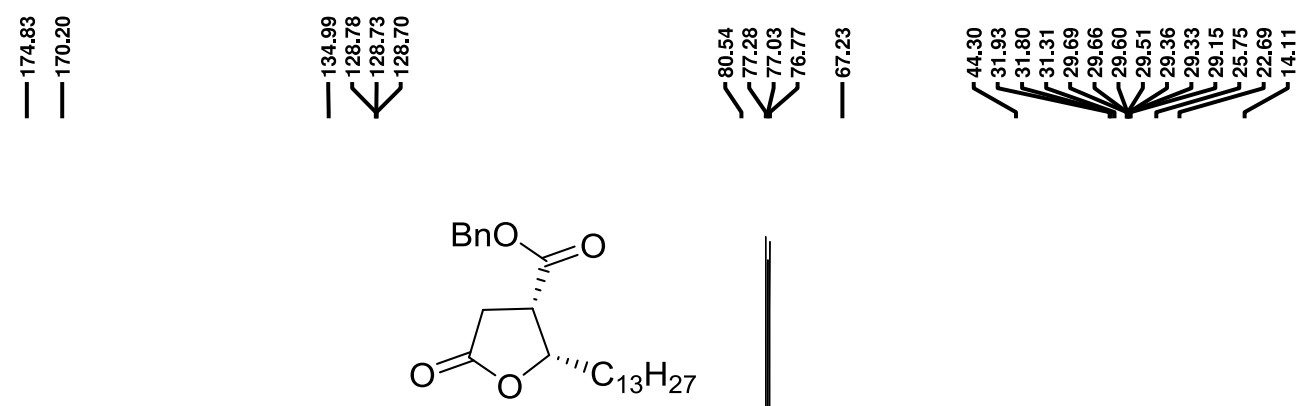

17c, $\mathrm{CDCl}_{3}, 125 \mathrm{MHz}$
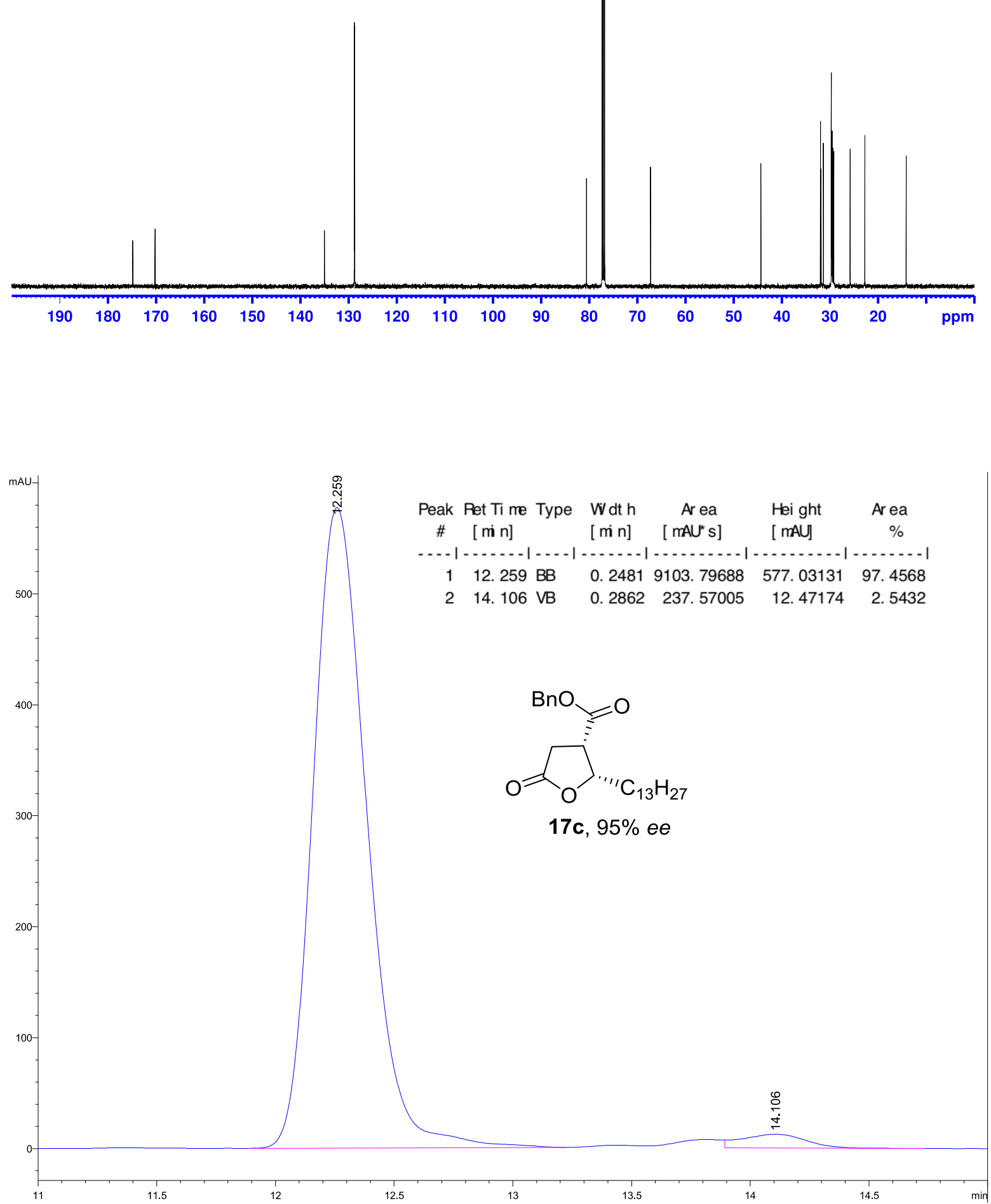


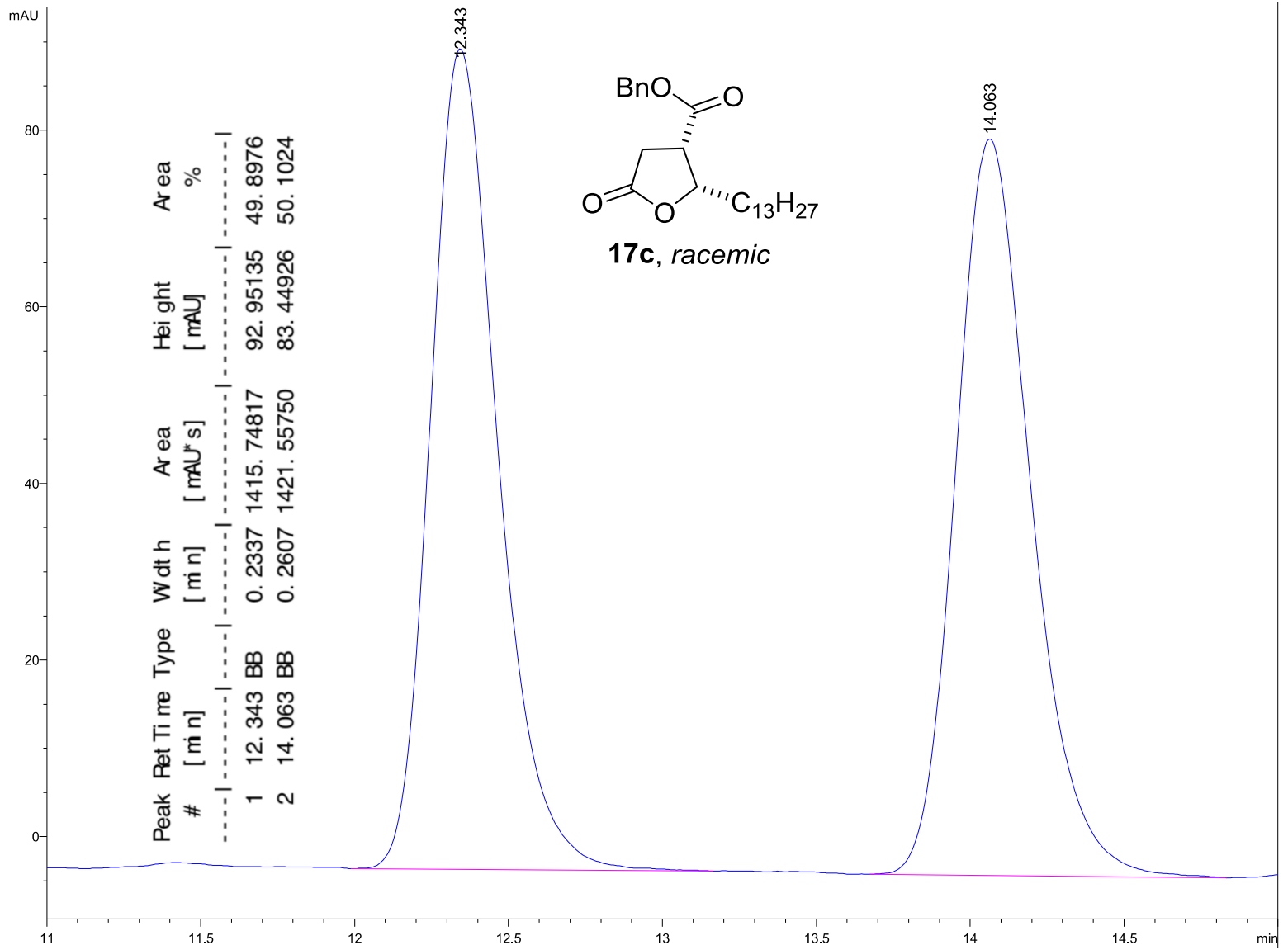

